\title{
WHEN DOES ALMOST FREE IMPLY FREE? (FOR GROUPS, TRANSVERSALS, ETC.)
}

\author{
MENACHEM MAGIDOR AND SAHARON SHELAH
}

\section{INTRODUCTION}

We say that a (Abelian) group is almost free if every subgroup of smaller cardinality is free. Suppose that $G$ is an almost free (Abelian) group. Is $G$ free? For Abelian groups a counterexample was given by R. Baer [Fu]. For groups, Higmann in [Hi] constructed a nonfree group of cardinality $\aleph_{1}$ such that every countable subgroup of it is free; hence it is almost free. For abelian groups the problem of "when does almost free imply free?" can be considered to be a generalization of Pontryagin's theorem (see [Fu]), claiming that a countable Abelian group is free if and only if every subgroup of it of finite rank is free. An excellent reference to most of the results mentioned in this introduction is the book by Eklof and Mekler [Ek-Me].

The Baer and Higmann counterexamples are of size $\aleph_{1}$. Work by Hill [Hill], Eklof [Ek] (for Abelian groups), and Mekler (for groups) [Me] showed that one can have such construction for $\aleph_{n}(n<\omega)$. Eklof (and independently Gregory and Shelah) actually proved a "pump up" lemma, by which a counterexample of cardinality $\kappa$ ( $\kappa$ regular) can be pushed up to $\lambda$ ( $\lambda$ regular), provided there exists a stationary subset of $\lambda, E$, of points of cofinality $\kappa$ which does not reflect; namely, $\forall \alpha<\lambda, E \cap \alpha$ is nonstationary in $\alpha$.

Since in the constructible universe (by a theorem of Jensen [Jen]) at every regular cardinal $\lambda$, which is not weakly compact, one can find a nonreflecting stationary set whose points are of any given cofinality $\kappa<\lambda$, it follows that in $L$ there is an almost free nonfree group (Abelian or not) at any regular cardinal which is not weakly compact. (As shown by Eklof "almost free" implies "free" for $\kappa$ which is weakly compact.)

What about singular cardinals? Can one find an almost free nonfree group of cardinality $\kappa$ when $\kappa$ is singular? The problem for Abelian groups was attacked by Hill who proved in [Hil2] that if $\kappa$ has cofinality $\aleph_{0}$ then every almost free Abelian group of cardinality $\kappa$ is free. (In [Hil3] he extended it to the case where the cofinality of $\kappa$ is $\aleph_{1}$.)

The major step was taken by Shelah in [Sh1] (see also [Ho]). Shelah realized

Received by the editors August 5, 1988.

1991 Mathematics Subject Classification. Primary 20A15, 20E05, 20K15, 20K20; Secondary $03 \mathrm{E} 10$

The first author's research was partially supported by U.S.-Israel Binational Science Foundation grant 87-00040, and the second author's by U.S.-Israel Binational Science Foundation grant 9000329. 
that Hill's theorem can be generalized to all singular cardinals, but more importantly the cases of groups and Abelian groups are just special cases of a much more general phenomenon. Shelah considers a general notion of "freeness"; namely, given a set $A$, one can consider a notion of "freeness" as a collection $\mathscr{F}$ of pairs of the form $(B, C)$ where $B, C \subseteq A$, where $(B, C) \in \mathscr{F}$ means intuitively " $B$ is free over $C$ ". (Being free means being free over the empty set.) $\mathscr{F}$ has to satisfy a certain set of axioms (which are very natural in the context of algebra on $A$, where $(B, C) \in \mathscr{F}$ if the subalgebra generated by $B, B^{*}$ is free over the subalgebra $B^{*} \cap C^{*}$ where $C^{*}$ is the subalgebra generated by $C$; "free" means free in the appropriate variety). This set of axioms has a cardinal parameter $\chi$ which for the example of free groups and free Abelian groups, etc. is $\aleph_{0}$. (For this set of axioms see $\S 2$ of this paper.)

Shelah proved a general compactness theorem for singular $\kappa$; namely, say that $A$ (with respect to the notion of "freeness" $\mathscr{F}$ ) is $\kappa$ free if every $B \subseteq$ $A$ of cardinality less than $\kappa$ is free. Shelah showed that if $\mathscr{F}$ satisfies the required axioms with some parameter $\chi<\kappa$, then $\kappa$ free implies $\kappa^{+}$free. (In particular, if $|A|=\kappa$, then $A$ is free if it is "almost free".) In particular if a group (Abelian group) is almost free and of cardinality $\kappa$, where $\kappa$ is singular, then it is free. This theorem is called the compactness theorem because it is the kind of theorem where properties of a "small" substructure of a structure imply a global property for the whole structure. Following this terminology we shall call a cardinal $\kappa \lambda$ compact if $\kappa$ free implies $\lambda^{+}$free for any notion of freeness satisfying the set of axioms described in $\S 2$ of this paper with parameter $\chi<\kappa . \kappa$ is fully compact if it is $\lambda$ compact for all $\lambda \geq \kappa$, and $\kappa$ is compact if it is $\kappa^{+}$compact. Hence by Shelah's theorem any singular $\kappa$ is compact.

There are many more examples satisfying Shelah's axioms than indicated by the examples of free varieties. For instance suppose that $A$ carries a graph structure, and let $\lambda<\kappa, \kappa$ singular. Define $(B, C) \in \mathscr{F}$ if $B, C \in A$ and $B-C$ can be well ordered so that every element is connected to less than $\lambda$ many elements preceding it in this well order. This notion of freeness satisfies Shelah's axioms; hence we get as a corollary that if every subgraph of $A$ of cardinality $<\kappa$ can be well ordered as above ( $=$ has coloring number $\lambda$ ), then $A$ has coloring number $\lambda$. Similarly let us consider the problem of transversals. Suppose that $A$ is a family of sets, each of them of cardinality $<\chi$ where $\chi<\kappa$. A transversal for $A$ is a one-to-one choice function on $A$. For $B, C \subseteq A$ we say $(B, C) \in \mathscr{F}$ if there is a one-to-one choice function on $B$ whose range is disjoint from $\cup B$. F satisfies Shelah's axioms with parameter $\chi$; hence if $|A|=\kappa$ and $\kappa$ is singular, and every subfamily of $A$ of cardinality $<\kappa$ has a transversal, then $A$ has a transversal. Since this property will be important in this paper we define $\operatorname{PT}(\kappa, \lambda)$ to mean: Given a family of $\kappa$ sets, each of which is of cardinality $<\lambda$ such that every subset of cardinality $<\kappa$ has a transversal, then the whole family has a transversal. If $\operatorname{PT}(\kappa, \lambda)$ fails (i.e., there is a counterexample) we denote this fact by $\operatorname{NPT}(\kappa, \lambda)$.

In [Sh4] it is shown that $\operatorname{NPT}\left(\kappa, \aleph_{1}\right)$ is equivalent to the existence of an almost free nonfree Abelian group of cardinality $\kappa$. Also $\operatorname{NPT}\left(\kappa, \aleph_{1}\right)$ implies the existence of an almost free nonfree group of cardinality $\kappa$. (The inverse direction is open.) 
Which regular cardinals can be compact in our sense? We already know that $\aleph_{n}(0<n<\omega)$ are not compact (since we have counterexamples which are Abelian groups). Similarly in $L$ no regular cardinal except weakly compacts is compact in our sense. As noted by Eklof, a weakly compact cardinal is compact in our sense, so if one accepts the consistency of weakly compact cardinals, one gets the consistency of a regular compact cardinal. (A much larger cardinal, supercompact, gives a fully compact cardinal.) But even a weakly compact cardinal is very large (strongly inaccessible, etc.). Can smaller regular cardinals be compact? The first cardinal we have to consider is $\aleph_{\omega+1}$. Shelah [Sh2] has shown that if $2^{\aleph_{0}}<\aleph_{\omega}$, then $\operatorname{NPT}\left(\aleph_{\omega+1}, \aleph_{1}\right)$; hence $\aleph_{\omega+1}$ is not compact under this cardinal arithmetic assumption. It will follow from the results of $\S 1$ of this paper that one can eliminate any cardinal arithmetic assumptions, so $\aleph_{\omega+1}$ is never compact. By Eklof's pumping up lemma (or alternatively using the Milner-Shelah pumping up for NPT [Mi-Sh], claiming that $\operatorname{NPT}(\kappa, \lambda)$ implies, for regular $\kappa, \operatorname{NPT}\left(\kappa^{+}, \lambda\right)$ ), one can show that $\aleph_{\omega+2}, \aleph_{\omega+3}, \ldots$ are not compact. Our arguments in $\S 1$ generalize to $\aleph_{\omega \cdot n+1}$. (This was done in [Sh2] under the assumption that $\aleph_{\omega \cdot n}$ is a strong limit.) Hence for every regular cardinal $\kappa$ less than $\aleph_{\omega^{2}}$ we have $\operatorname{NPT}\left(\kappa, \aleph_{1}\right)$, and so we have an Abelian group of cardinality $\kappa$ which is almost free but not free. Since the corresponding notions of freeness satisfy the axioms from [Sh1], we get that there is no compact cardinal below $\aleph_{\omega^{2}}$.

Thus the next regular cardinal we have to consider is $\aleph_{\omega^{2}+1}$. In $\S 3$ of this paper we shall show that assuming the consistency of infinitely many supercompact cardinals, one can get a model of $\mathrm{ZFC}+$ G. C. H. + " $\aleph_{\omega^{2}+1}$ is compact". In particular in this model an almost free (Abelian) group of cardinality $\aleph_{\omega^{2}+1}$ is free. The method of $\S 3$ can be easily adapted to show that (under the same assumption) for $\alpha<\omega_{1}$ one can get a model of ZFC + G. C. H. $+{ }^{“} \aleph_{\omega^{2}}$ free implies $\aleph_{\alpha}$ free". (In particular, $\aleph_{\omega^{2}+1}$ is $\aleph_{\beta}$ compact for $\beta \leq \alpha$. )

Can $\aleph_{\omega_{2}+1}$ be fully compact? If not, which cardinal can be the first fully compact cardinal? In $\S 1$ we show that $\aleph_{\omega^{2}+1}$ cannot be fully compact. There we prove that $\operatorname{NPT}\left(\aleph_{\omega_{1}+1}, \aleph_{1}\right)$. (Also NPT $\left(\aleph_{\omega_{1}+n}, \aleph_{1}\right)$ for $0<n<\omega$.) In general we show that if $\kappa$ is regular, $\kappa<\aleph_{\kappa}$, then $\operatorname{NPT}\left(\aleph_{\kappa+1}, \kappa\right)$. We are more interested in $\operatorname{NPT}\left(\aleph_{\kappa+1}, \aleph_{1}\right)$, but, if we can show that $\operatorname{NPT}\left(\kappa, \aleph_{1}\right)$ holds, we can get $\operatorname{NPT}\left(\aleph_{\kappa+1}, \aleph_{1}\right)$. As usual [Sh4] implies that if $\kappa$ carries an almost free nonfree Abelian group, then one can get such a group of cardinality $\aleph_{\kappa+1}$. So we get that $\aleph_{\omega_{1}+1}$ is not compact (and the counterexample can be assumed to be an Abelian group). Similarly if we define inductively $\kappa_{0}=\omega_{1}, \kappa_{n+1}=\aleph_{\kappa_{n}+1}$ we get that each $\kappa_{n}$ is not compact. So there is no fully compact cardinal below $\sup _{n<\omega} \kappa_{n} . \sup _{n<\omega} \kappa_{n}$ is the first cardinal fixed point, i.e., the first $\kappa$ such that $\kappa=\aleph_{\kappa}$. Again, the counterexample to compactness is an Abelian group, so below the first cardinal fixed point we get arbitrarily large cardinals carrying an almost free nonfree Abelian group.

So which cardinal can be the first fully compact? In $\S 4$ we show (under the assumption of infinitely many supercompacts) that one can have a model of ZFC + G.C.H. + "the first cardinal fixed point is fully compact". So the 
first cardinal fixed point is the smallest cardinal which can consistently be fully compact. In particular (assuming the consistency of $\omega$ many supercompacts), one cannot prove in set theory that there are almost free nonfree Abelian groups whose cardinality is above the first cardinal fixed point.

The structure of the paper should be clear by now, except for $\S 2$, where sufficient conditions are given for $\lambda$ compactness (or full compactness). These sufficient conditions will be used in $\S \S 3$ and 4 to verify, in the appropriate models, that $\aleph_{\omega^{2}+1}$ and the first cardinal fixed point are compact and fully compact respectively. In $\S 2$ we introduce two reflection principles $\Delta_{\kappa}$, and $\Delta_{\kappa}^{-}$which will be used to prove the full compactness of $\kappa . \Delta_{\kappa}$ is a stronger principle, and in some sense it is more natural; however, when in $\S 4$ we get a model in which the first cardinal fixed point is fully compact, we are not able to prove that $\Delta_{\kappa}$ holds in that model (though the weaker $\Delta_{\kappa}^{-}$is heavily used). We think that $\Delta_{\kappa}$ is of independent interest. By a construction which is similar to the construction of $\S 4$ we are able to get a model in which the first cardinal fixed point of second order (namely, the first $\kappa$ having $\kappa$ many fixed points below it) satisfies $\Delta_{\kappa}$. This modified construction will be published in a later paper.

Our notation and terminology are (hopefully) standard (see [Je]). $V^{P}$ is a generic extension of the model $V$, using $P$ as the forcing notion. The corresponding generic filter will be $G_{P}$. We also assume familiarity with basic facts about supercompact cardinals and normal ultrafilters on $P_{\kappa}(\lambda)$ (see [S$\mathrm{R}-\mathrm{K}]$ or $[\mathrm{K}-\mathrm{M}]$ ). (Actually no forcing or large cardinals are needed for reading $\S \S 1$ and 2. Especially for $\S 2$ the set theoretical requirements are minimal.) For iterated forcing terminology see [Sh3] or [Ba2]. Each section will have its own numbering for theorems, lemmas, etc., where cross-reference will be denoted by "Theorem 2.1", etc.

\section{MORE CASES OF INCOMPACTNESS}

In this section we prove in ZFC that many cardinals are not compact. In particular, we show that the cardinals for which our theorem applies carry an almost free nonfree Abelian group. Some of the results we give here were known before under some cardinal arithmetic assumptions (for instance, for $\aleph_{\omega+1}$ ) assuming that $2^{\aleph_{0}}<\aleph_{\omega}$; see [Sh2], and here we prove it without any assumptions. For other cardinals their incompactness was not known before. Actually in the first version of this paper these incompactness results were proved using some weak version of G. C.H. (For instance in Theorem 4 below we had to assume $2^{\kappa}<\lambda$, so in Definition 2 below we did not have to worry about case III. When the second author made progress in PCF theory (see [Sh5]) the cardinal arithmetic assumptions could be eliminated.)

Recall from the introduction that $\operatorname{NPT}(\lambda, \kappa)$ means that there is a sequence of sets indexed by $\lambda$, each of them of cardinality less than $\kappa$ such that the family is almost free but not free. Namely, every subfamily of cardinality less than $\lambda$ has a transversal, but the whole family does not have a transversal. For instance we have $\operatorname{NPT}\left(\aleph_{0}, \aleph_{1}\right)$. (Take as the family $\mathscr{F}=\{\{n\} \mid n \in \omega\} \cup\{\omega\}$. ) $\operatorname{By}[\operatorname{Sh} 4] \operatorname{NPT}\left(\lambda, \aleph_{1}\right)$ is equivalent (for $\lambda>\aleph_{0}$ ) to $\lambda$ carrying an almost free 
nonfree Abelian group. The main theorem of this section is

Theorem 1. Let $\mathscr{C}_{\delta}$ be the smallest set of cardinals containing the regular cardinal $\delta$ and closed under taking successor cardinals and under the operation $(\lambda, \kappa) \rightarrow \lambda^{+\kappa+1}$. Assume that $\operatorname{NPT}\left(\delta, \aleph_{1}\right)$ holds. Then for every $\lambda \in \mathscr{C}_{\delta}$ we have $\operatorname{NPT}\left(\lambda, \aleph_{1}\right)$.

Corollary 2. For every $n, m<\omega, m>0$ we have $\operatorname{NPT}\left(\aleph_{\omega \cdot n+m}\right)$. Hence we have an almost free nonfree Abelian group in every uncountable regular cardinality below $\aleph_{\omega^{2}+1}$. In particular, there is no regular compact cardinal below $\omega^{2}+1$.

Proof. We have $\mathrm{NPT}\left(\aleph_{0}, \aleph_{1}\right)$. All the cardinals mentioned in the corollary are in $\mathscr{C}_{\aleph_{0}}$.

Corollary 3. If there is an almost free nonfree Abelian group of cardinality $\kappa$, then there exists one of cardinality $\aleph_{\kappa+1}$. Hence there are arbitrarily large almost free nonfree Abelian groups below the first cardinal fixed point. In particular, there is no fully compact cardinal below the first cardinal fixed point.

Proof. The first claim follows immediately from Theorem 1 for $\lambda=\aleph_{1}$ and $\kappa$. The second claim follows from the first by defining by induction a sequence of cardinals $\kappa_{n}$ for $n<\omega$.

$$
\kappa_{0}=\aleph_{1}, \quad \kappa_{n+1}=\aleph_{\kappa_{n}} .
$$

By the first claim each $\kappa_{n}$ carries an almost free nonfree Abelian group, and clearly the supremum of the $\kappa_{n}$ 's is the first cardinal fixed point.

For the sequel we need the following generalization of $\operatorname{NPT}(\kappa, \lambda)$ :

Definition 1. Let $A$ be a set and $I$ an ideal on $A$. A family of sets $\left\langle B_{a} \mid a \in A\right\rangle$ is said to be $I$ free if for every $C \subseteq A, C \in I$, the family $\left\langle B_{a} \mid a \in C\right\rangle$ has a transversal. $\operatorname{NPT}(A, I, \kappa)$ holds if there exists a family of sets of cardinality less than $\kappa$, indexed by $A$, which is $I$ free but not free. In case that the set $A$ is clearly fixed by the ideal $I$, we shall write $\operatorname{NPT}(I, \kappa)$ for $\operatorname{NPT}(A, I, \kappa)$.

Note that $\operatorname{NPT}(\lambda, \kappa)$ is exactly $\operatorname{NPT}\left(\lambda, I_{<\lambda}, \kappa\right)$, where $I_{<\lambda}$ is the ideal of sets of cardinality less than $\lambda$. Note also that an example for $\operatorname{NPT}(\lambda, I, \kappa)$ is also an example for $\operatorname{NPT}(\lambda, J, \kappa)$ for every ideal $J$ included in $I$.

If $S$ is a stationary subset of $\lambda$, we let $N S_{S}$ be the nonstationary ideal restricted to $S$. If $\vec{S}$ is a finite sequence of sets and $\vec{\kappa}=\kappa_{0} \cdots \kappa_{n}$ is a finite sequence of regular cardinals such that $S_{i}$ is a stationary subset of $\kappa_{i}$, we denote by $I_{\vec{S}}$ the ideal on $\Pi \vec{\kappa}$ which is the product of the ideals $N S_{S_{i}}$. Recall that the product $I \times J$ of two ideals $I$ and $J$, where $I$ is on $A$ and $J$ is on $B$, is the ideal defined on $A \times B$ by $Z \subseteq A \times B, Z \in I \times J$ iff $\{b \mid\{a \mid(a, b) \in Z\} \in I\} \in J$.

The main technical tool for proving Theorem 1 is the following theorem, which is of interest by itself.

Theorem 4. Let $\kappa, \lambda$ be regular cardinals, $\kappa<\lambda$. Let $\tau=\lambda^{+\kappa+1}$. Then there exist a stationary subset of $\tau, S$, of points of cofinality $\lambda$ and a sequence of sets $\left\langle A_{\alpha} \mid \alpha \in S\right\rangle$ such that, for $\alpha \in S, A_{\alpha}$ is a subset of $\lambda^{+\kappa}$ of order type $\kappa$ such that for every nonstationary subset of $S, T$, one can find $\left\langle D_{\alpha} \mid \alpha \in T\right\rangle$ and 
$\left\langle B_{\alpha} \mid \alpha \in T\right\rangle$ such that, for $\alpha \in T, D_{\alpha}$ is a closed unbounded subset of $\alpha$ and $B_{\alpha}$ is a subset of $A_{\alpha}$ satisfying $\left|A_{\alpha}-B_{\alpha}\right|<\kappa$ and the sets $\left\langle B_{\alpha} \times D_{\alpha} \mid \alpha \in T\right\rangle$ are mutually disjoint.

Theorem 4 immediately gives examples of the failure of the transversal property. As an example we have

Corollary 5. Let $\kappa$ and $\lambda$ be as in Theorem 4; then $\operatorname{NPT}\left(\lambda^{+\kappa+1}, \lambda^{+}\right)$.

Proof. Let $S,\left\langle A_{\alpha} \mid \alpha \in S\right\rangle$ be the witnesses to Theorem 4. For each $\alpha \in S$ pick $E_{\alpha}$ which is a closed unbounded subset of $\alpha$ of order type $\operatorname{cf}(\alpha)=\lambda$. The family of sets $\left\langle E_{\alpha} \times A_{\alpha} \mid \alpha \in S\right\rangle$ is an example of $\operatorname{NPT}\left(\lambda^{+\kappa+1}, \lambda^{+}\right)$. This family is clearly of cardinality $\lambda^{+\kappa+1}$ and every member of it is of cardinality $\lambda$. This family does not have a transversal because such a transversal will be essentially a function $F$ defined on $S$ such that, for $\alpha \in S, F(\alpha) \in \alpha \times \lambda^{+\kappa}$. Easy application of Fodor's lemma shows that $F$ is constant on a stationary subset of $S$, so $F$ cannot be one-to-one. Every subfamily of smaller cardinality does have a transversal. Without loss of generality the smaller cardinality subset is $\left\langle E_{\alpha} \times A_{\alpha} \mid \alpha \in S \cap \beta\right\rangle$ for some $\beta<\lambda^{+\kappa+1}$. Let $\left\langle D_{\alpha} \mid \alpha \in S \cap \beta\right\rangle$ and $\left\langle B_{\alpha} \mid \alpha \in S \cap \beta\right\rangle$ be as in the conclusion of Theorem 4. If we let $F(\alpha)$, for $\alpha \in S \cap \beta$, be any pair of the form $(\gamma, \delta)$, where $\delta \in B_{\alpha}$ and $\gamma \in E_{\alpha} \cap D_{\alpha}$, then $F$ is a one-to-one choice function on the given subfamily.

Proof of Theorem 1 from Theorem 4 . Let $\mathscr{C}=\mathscr{C}_{\delta}$. By induction on the cardinals $\tau \in \mathscr{C}$ we prove the following strengthening of Theorem 1 .

Claim. Assume $\operatorname{NPT}\left(\delta, \aleph_{1}\right)$. For every finite increasing sequence of cardinals in $\mathscr{C}-\{\delta\}, \vec{\mu}$, there exists another finite increasing sequence in $\mathscr{C}-\{\delta\}, \vec{\rho}$, containing all the members of $\vec{\mu}$ and having the same maximum, and there exists a sequence of sets $\vec{S}$ such that $S_{i}$ is a stationary subset of $\rho_{i}$ such that we have $\operatorname{NPT}\left(I, \aleph_{1}\right)$, where $I$ is the ideal $I_{<\delta} \times I_{\vec{S}}$.

The proof of the claim is by induction on the maximal member $\tau$ of $\{\delta\} \cup \vec{\mu}$. The initial case $\tau=\delta$ is vacuously true since we assumed $\operatorname{NPT}\left(\delta, \aleph_{1}\right)$. If $\tau=\mu^{+}$, where $\mu \in \mathscr{C}$, then we can invoke [Mi-Sh]. In that paper one starts with an example for $\operatorname{NPT}\left(\mu, \aleph_{1}\right)$ and "pumps it up" to an example for $\operatorname{NPT}\left(\tau, \aleph_{1}\right)$. The particular example constructed in [Mi-Sh] is indexed by the set of pairs $T=\{(\alpha, \beta) \mid \alpha<\tau, \operatorname{cf}(\alpha)=\mu, \beta<\mu\}$. Their proof actually shows that if the example for $\mu$ is actually an example for $\operatorname{NPT}\left(\mu, I, \aleph_{1}\right)$ for some ideal $I$ on $\mu$, then, for every subset of $T, U$ such that

$$
\{\alpha<\tau \mid\{\beta<\mu \mid(\alpha, \beta) \in U\} \notin I\}
$$

is not stationary in $\tau$, the subfamily of sets with indices in $U$ has a transversal (which by our terminology means that this is an example for $\operatorname{NPT}\left(\mu, I \times N S_{S}, \aleph_{1}\right)$ where $S$ is the stationary subset of $\tau$ of points of cofinality $\mu)$.

Given a finite sequence of cardinals in $\mathscr{C}, \vec{\eta}$, all of them less than $\tau$ and above $\delta$, we shall verify the claim for the sequence $\vec{\eta}^{\uparrow}\{\tau\}$. One can assume without loss of generality that $\mu$ appears in $\vec{\eta}$, and, by the induction assumption, we have a sequence of stationary sets $\vec{S}\left(S_{i}\right.$ a stationary subset of $\eta_{i}$ such 
that we have $\operatorname{NPT}\left(I, \aleph_{1}\right)$, where $I$ is the ideal $\left.I_{<\delta} \times I_{\vec{S}}\right) . I$ can be considered to be an ideal on $\mu$, so applying the Milner-Shelah construction to this example, we get an example of $\operatorname{NPT}\left(I^{*}, \aleph_{1}\right)$, where $I^{*}$ is the ideal $N S_{\{\alpha \mid \alpha<\tau, \operatorname{cf}(\alpha)=\mu\}} \times I$; thus we proved the claim for this case.

The second case is when $\tau=\lambda^{+\kappa+1}$ where the induction assumption was already verified for $\kappa$ and $\lambda$. Without loss of generality we can assume that $\kappa<\lambda$, because if $\lambda<\kappa$ we can replace $\lambda$ by $\kappa^{+}$. The value of $\tau$ will not be changed by it. (Note that all the cardinals in our set $\mathscr{C}$ are less than the first cardinal fixed point above $\delta$.) We are also given a finite sequence of cardinals below $\tau, \vec{\rho}$; all of the members are in $\mathscr{C}-\{\delta\}$. Again without loss of generality we can assume that $\kappa$ appears in $\vec{\rho}$ (or $\kappa=\delta$ ) and that $\lambda$ is the maximal member of $\vec{\rho}$ by replacing $\lambda$ with the maximal member of $\vec{\rho}$, if necessary. By the induction assumption we can also assume that for some sequence of sets $\vec{S}$, such that $S_{i}$ is a stationary subset of $\rho_{i}$, we have $\operatorname{NPT}\left(I, \aleph_{1}\right)$ where $I$ is $I_{\vec{S}} \times I_{<\delta}$. Fix a sequence of countable sets $\left\langle X_{\vec{\eta}} \mid \vec{\eta} \in \delta \times \prod \vec{S}\right\rangle$ which is an example of $\operatorname{NPT}\left(I, \aleph_{1}\right)$. Let $S$ and $\left\langle A_{\alpha} \mid \alpha \in S\right\rangle$ be witnesses to the truth of Theorem 4. Let $a(\alpha, \gamma)$, for $\gamma \in \kappa$, be the $\gamma$ th member of $A_{\alpha}$. We shall verify that if we extend $\vec{S}$ by the stationary subset of $\tau, S$, we will be able to prove $\operatorname{NPT}\left(I^{*}, \aleph_{1}\right)$, where $I^{*}$ is the ideal $I \times N S_{S}$, and our claim will be verified also in this case.

For each $\alpha \in S$ fix a closed unbounded subset of $\alpha$ of order type $\lambda, E_{\alpha}$. For $\alpha \in S$ and $\gamma \in \lambda$ let $e(\alpha, \gamma)$ be the $\gamma$ th member of $E_{\alpha}$ in the monotone enumeration of $E_{\alpha}$.

The family of countable sets that will witness $\operatorname{NPT}\left(\tau, I^{*}, \aleph_{1}\right)$ is $Y_{\vec{\eta}}$, where $\vec{\eta} \in \delta \times \prod \vec{S} \times S$. For $\vec{\eta} \in \delta \times \prod \vec{S} \times S$, let $\lambda(\vec{\eta}), \tau(\vec{\eta})$, and $\kappa(\vec{\eta})$ be the members of $\vec{\eta}$ corresponding to $\lambda, \tau$, and $\kappa$, respectively, and let $\vec{\eta}$ be $\vec{\eta}$ with its last coordinate (namely, the coordinate corresponding to $\tau$ ) omitted. We define

$$
Y_{\vec{\eta}}=X_{\vec{\eta}^{*}} \times\{\tau(\vec{\eta})\} \cup\left\{\left\langle\vec{\eta}^{*}, a(\tau(\vec{\eta}), \kappa(\vec{\eta})), e(\tau(\vec{\eta}), \lambda(\vec{\eta}))\right\rangle\right\} .
$$

We assume, without loss of generality, that the members of $X_{\vec{\eta}^{*}}$ are not a finite sequence of ordinals, so that the union in the definition of $Y_{\vec{\eta}}$ is a disjoint union. $Y_{\vec{\eta}}$ is clearly a countable set. The family of sets $Y_{\vec{\eta}}$ has no transversal, because if $\mathscr{F}$ is such a transversal, then for every $\alpha \in S$ there exists $\vec{\eta}_{\alpha}$ with $\tau\left(\vec{\eta}_{\alpha}\right)=\alpha$ such that

$$
\mathscr{F}\left(\vec{\eta}_{\alpha}\right)=\left\langle\vec{\eta}^{*}, a(\tau(\vec{\eta}), \kappa(\vec{\eta})), e(\tau(\vec{\eta}), \lambda(\vec{\eta}))\right\rangle .
$$

(Otherwise one can use $\mathscr{F}$ to define a transversal for $\left\langle X_{\vec{\eta}} \mid \vec{\eta} \in \delta \times \Pi \vec{S}\right\rangle$.) But an easy application of Fodor's lemma for the stationary set $S$ will get $\alpha$ and $\alpha^{\prime}$ in $S\left(\alpha \neq \alpha^{\prime}\right)$ such that

$$
\mathscr{F}\left(\vec{\eta}_{\alpha}\right)=\mathscr{F}\left(\vec{\eta}_{\alpha^{\prime}}\right)
$$

which is of course a contradiction.

We are left with the task of verifying that $W$ is a set in the ideal $I^{*}$; then the family $\left\langle Y_{\vec{\eta}} \mid \vec{\eta} \in W\right\rangle$ has a transversal. For $\alpha \in S$ put

$$
W_{\alpha}=\{\vec{\eta} \mid \vec{\eta} \in W, \tau(\vec{\eta})=\alpha\} .
$$


By definition of $I^{*}$ the set

$$
T=\left\{\alpha \mid \alpha \in S, W_{\alpha} \notin I\right\}
$$

is not stationary in $\tau$. We are ready to use the fact that $S$ and the sequence $\left\langle A_{\alpha} \mid \alpha \in S\right\rangle$ witness the truth of Theorem 4. Hence we can find $\left\langle B_{\alpha} \mid \alpha \in T\right\rangle$ and $\left\langle D_{\alpha} \mid \alpha \in T\right\rangle$ such that all the clauses of Theorem 4 are satisfied. Without loss of generality we can assume that, for $\alpha \in T, D_{\alpha} \subseteq E_{\alpha}$. By definition of the ideal $I$ it is clear that, for $\alpha \in T$,

$$
Q_{\alpha}=\left\{\vec{\eta}^{*} \mid \vec{\eta} \in W_{\alpha}, a(\alpha, \kappa(\vec{\eta})) \notin B_{\alpha}, \text { or } e(\alpha, \lambda(\vec{\eta})) \notin D_{\alpha}\right\} \in I .
$$

For $\alpha \in S-T$ put $Q_{\alpha}=W_{\alpha}$, so that we have $Q_{\alpha} \in I$ for every $\alpha \in S$. Since $\left\langle X_{\vec{\xi}} \mid \vec{\xi} \in \delta \times \prod \vec{S}\right\rangle$ was a witness to $\operatorname{NPT}\left(I, \aleph_{1}\right)$, we can find for each $\alpha \in S$ a transversal $\mathscr{F}_{\alpha}$ for $\left\langle X_{\vec{\xi}} \mid \xi \in Q_{\alpha}\right\rangle$. The transversal $\mathscr{F}$ for the set $\left\{Y_{\vec{\eta}} \mid \vec{\eta} \in W\right\}$ will be defined by (where $\alpha=\tau(\vec{\eta})$ )

$$
\mathscr{F}(\vec{\eta})=\left\langle\mathscr{F}_{\alpha}\left(\vec{\eta}^{*}\right), \alpha\right\rangle
$$

for $\vec{\eta} \in Q_{\alpha}$ and

$$
\mathscr{F}(\vec{\eta})=\left\langle\vec{\eta}^{*}, a(\alpha, \kappa(\vec{\eta})), e(\alpha, \lambda(\vec{\eta}))\right\rangle
$$

if $\vec{\eta} \notin Q_{\alpha}$. Note that in the second case we must have $\alpha \in T$ and $\mathscr{F}(\vec{\eta}) \in$ $\left\{\vec{\eta}^{*}\right\} \times B_{\alpha} \times D_{\alpha}$. It can now be easily verified that $\mathscr{F}$ is a one-to-one choice function on $W$, using the facts that $\mathscr{F}_{\alpha}$ is one-to-one on $Q_{\alpha}$ and that the family of sets $\left\{B_{\alpha} \times D_{\alpha} \mid \alpha \in T\right\}$ is a family of mutually disjoint sets.

Proof of Theorem 4. Let $\lambda, \kappa$, and $\tau$ be as in the statement of Theorem 4. Our main tool will be the study of reduced products of sequences of regular cardinals done by the second author ("The PCF theory"; see [Sh5, B-M]). It follows easily from the PCF theory (for instance using Theorem 2.1 in [Sh5] or Corollaries 2.2 and 4.4 in [B-M]) that there is a sequence $\left\langle\lambda_{i} \mid i<\kappa\right\rangle$ of regular cardinals cofinal in $\lambda^{+\kappa}$ (all of them above $\lambda$ ) such that the reduced product $\prod_{i<\kappa} \lambda_{i} / I_{<\kappa}$ has true cofinality $\tau$. Namely, there is an increasing sequence $\left\langle f_{\alpha} \mid \alpha<\tau\right\rangle$, in $\prod_{i<\kappa} \lambda_{i} / I_{<\kappa}$, which is also cofinal. Recall that a function $f$ in $\prod_{i<\kappa} \lambda_{i}$ is the least upper bound of $B \subseteq \prod_{i<\kappa} \lambda_{i} / I_{<\kappa}$ if $g<_{I} f$ for every $g \in B$, and if $g<_{I} f$, then, for some $h \in B, g<_{I} h$. (For the rest of this proof $I$ stands for $I_{<\kappa}$.) Without loss of generality we can assume that, for all $\alpha<\tau$, if there is a l.u.b. for $\left\{f_{\beta} \mid \beta<\alpha\right\}$, then $f_{\alpha}$ is this l.u.b. (It is unique up to equivalence modulo $I$.)

We shall classify the limit ordinals $\alpha \in \tau$ according to the behavior of the sequence $\left\langle f_{\beta} \mid \beta<\alpha\right\rangle$.

Definition 2. I. $\alpha<\tau$ is good (or of the first kind) if $f_{\alpha}$ is the l.u.b. of $\left\langle f_{\beta}\right| \beta<$ $\alpha\rangle$ and the function $g(i)=\operatorname{cf}\left(f_{\alpha}(i)\right)$ is constant modulo $I$, where this constant value is greater than $\kappa$. (Note that in this case every l.u.b. for $\left\langle f_{\beta} \mid \beta<\alpha\right\rangle$ has a fixed cofinality modulo $I$.)

II. $\alpha<\tau$ is bad (or of the second kind) if there is a sequence of sets of ordinals $\left\langle s_{i} \mid i<\kappa\right\rangle$ and an ultrafilter $D$ on $\kappa$ such that, for $i \in \kappa,\left|s_{i}\right|<\lambda, D$ is disjoint from $I$ (i.e., $D$ is uniform), and $\prod_{i<\kappa} s_{i}$ cuts $\left\langle f_{\beta} \mid \beta<\alpha\right\rangle$ cofinally. 
(Namely, for every $\beta<\alpha$ there exist $g \in \prod_{i<\kappa} s_{i}$ and $\beta<\gamma<\alpha$ such that $f_{\beta}<_{D} g<_{D} f_{\gamma}$. Note that without loss of generality we can assume that for all $i<\kappa$ we have $s_{i} \subseteq f_{\alpha}(i)$.)

III. $\alpha<\tau$ is chaotic (or of the third kind) if it is not bad and there exists a function $g \in \prod_{i<\kappa} \lambda_{i} / I_{<\kappa}$ such that the sequence of sets $t_{\beta}=\{i \mid i<\kappa$, $\left.f_{\beta}(i)<g(i)\right\}$ is positive modulo $I$ for $\beta<\alpha$, but it is not fixed modulo $I$. (We clearly have, for $\beta<\gamma<\alpha, t_{\beta} \subseteq_{I} t_{\gamma}$ since the $f_{\beta}$ 's are increasing modulo $I$, but in this case we also have that for every $\beta<\alpha$ there is $\gamma<\alpha$ such that $\left.t_{\beta} \subset_{I} t_{\gamma}.\right)$

The following lemma is a slight variation of Claim 1.2 of [Sh5, Chapter 2].

Lemma 5. I. For every $\alpha \in \tau, \operatorname{cf}(\alpha)>\lambda, \alpha$ is either good or bad or chaotic (and exactly one of these cases holds).

II. For $\alpha \in \tau, \operatorname{cf}(\alpha)=\lambda$; then, if $\alpha$ is good, $\alpha$ is neither bad nor chaotic.

III. If $\alpha<\tau$ has cofinality bigger than $\lambda$, then, if $\alpha$ is good (respectively, bad, chaotic), there is a closed unbounded subset of $\alpha, C$, such that for $\beta$ in $C, \operatorname{cf}(\beta)>\kappa, \beta$ is good (respectively, bad, chaotic).

Proof. A basic observation that will be used also later is

Lemma 6. If $\alpha<\tau, \operatorname{cf}(\alpha)>\kappa$; then $\alpha$ is good iff there exist a cofinal subset of $\alpha, B$, and $A \in I$ such that for $i \notin A, \beta<\gamma$ in $B$, we have

$$
f_{\beta}(i)<f_{\gamma}(i) \text {. }
$$

Proof of Lemma 6. Assume that $\alpha<\tau$ is good. Let $\mu$ be such that $\operatorname{cf}\left(f_{\alpha}(i)\right)$ is constantly $\mu$ modulo $I$. By definition of "good" we have $\mu>\kappa$. Without loss of generality assume that, for all $i \in \kappa, \operatorname{cf}\left(f_{\alpha}(i)\right)=\mu$. For each $i \in \kappa$ fix a cofinal subset of $f_{\alpha}(i)$ of order type $\mu$ and denote it by $E_{i}$. For $\delta \in \mu$ let $h_{\delta} \in \prod_{i<\kappa} \lambda_{i} / I_{<\kappa}$ be defined by

$$
h_{\delta}(i)=\text { the } \delta \text { th member of } E_{i} .
$$

We claim that if $g \in \prod_{i<\kappa} \lambda_{i} / I_{<\kappa}$ satisfies $g<_{I} f_{\alpha}$, then there is $\delta<\mu$ such that $g<{ }_{I} h_{\delta}$. Without loss of generality we can assume that $g(i)<f(i)$ for every $i<\kappa$. For each $i<\kappa$ let $\rho_{i}$ be an ordinal less than $\mu$ such that $g(i)$ is less than the $\rho_{i}$ th member of $E_{i}$. Let $\rho<\mu$ be an upper bound for $\rho_{i}$, $i<\kappa$. Clearly we have $g<_{I} h_{\rho}$. Since also the set $\left\{f_{\beta} \mid \beta<\alpha\right\}$ is cofinal in the set $\left\{g \in \prod_{i<\kappa} \lambda_{i} / I_{<\kappa} \mid g<_{I} f_{\alpha}\right\}$, we get $\mu=\operatorname{cf}(\alpha)$.

By the previous remark, for each $\beta<\alpha$ we can find $\delta(\beta)<\mu$ such that $f_{\beta}<{ }_{I} h_{\delta(\beta)}$. Also $f_{\alpha}$ is the 1.u.b. of $\left\langle f_{\beta} \mid \beta<\alpha\right\rangle$; hence for every $\delta<\mu$ we can find $\beta<\alpha$ such that $h_{\delta}<_{I} f_{\beta}$. Using the last two observations, one can define by induction an increasing sequence $\left\langle\beta_{i} \mid i<\mu\right\rangle$, cofinal in $\alpha$, such that for $i<j<\mu$

$$
f_{\beta_{i}}<h_{I} h_{\delta\left(\beta_{i}\right)}<_{I} f_{\beta_{j}} .
$$

For $i \in \mu$ let $B_{i}$ be the set in $I$ such that for $k \notin B_{i}$

$$
f_{\beta_{i}}(k)<h_{\delta\left(\beta_{i}\right)}(k)<f_{\beta_{i+1}}(k) .
$$

The ideal $I$ is generated by $\kappa$ sets and $\mu>\kappa$; hence we can find $A \in I$ such that the set $D=\left\{i+1 \mid i<\mu, B_{i+1} \subseteq A\right\}$ is unbounded in $\mu$. We claim 
that the set $A$ and the set $\left\{\beta_{i+1} \mid i+1 \in D\right\}$ are witnesses to the claim of the present lemma. If $i+1<j+1$ are two members in $D$, then for $k \notin A$ we have

$$
f_{\beta_{i+1}}(k)<h_{\delta\left(\beta_{i+1}\right)}(k) \leq h_{\delta\left(\beta_{j}\right)}(k)<f_{\beta_{j+1}}(k)
$$

where for the second inequality we used the fact that if $\delta<\gamma$ then $h_{\delta}(k)<$ $h_{\gamma}(k)$ for every $k$. We have verified one direction of the lemma.

For the other direction of the lemma, let $A \in I$ and $B \subseteq \alpha$ be as in the statement of the lemma. Without loss of generality the order type of $B$ is $\mu=\operatorname{cf}(\alpha)$. Let $f \in \prod_{i<\kappa} \lambda_{i} / I_{<\kappa}$ be defined by $f(i)=\sup \left\{f_{\beta}(i) \mid \beta \in B\right\}$. By assumption, for $i \notin A, \operatorname{cf}(f(i))=\mu$. We claim that $f$ is a l.u.b. for $\left\langle f_{\beta} \mid \beta<\alpha\right\rangle$. It is clearly a bound for every $f_{\beta}$ for $\beta \in B$. Hence it is an upper bound for $\left\langle f_{\beta} \mid \beta<\alpha\right\rangle$, since $B$ is cofinal in $\alpha$. Let $g<_{I} f$. Without loss of generality $g(i)<f(i)$ for every $i \in \kappa$. By definition of $f$, for every $i \notin A$, we can find $\beta_{i} \in B$ such that $g(i)<f_{\beta_{i}}(i)$. Let $\beta=\sup \left\{\beta_{i} \mid i<\kappa\right\}$. Since $\mu>\kappa$, we have $\beta<\alpha$ and by the assumption about $A$ and $B$ we have, for $i \notin A$,

$$
g(i)<f_{\beta}(i)
$$

which proves that $f$ is the least upper bound for $\left\langle f_{\beta} \mid \beta<\alpha\right\rangle$. Since $f_{\alpha}$ was picked to be a l.u.b. for $\left\langle f_{\beta}\right| \beta\langle\alpha\rangle$, if one exists, $f_{\alpha}$ is such a l.u.b., and it must be equivalent to $f$ modulo $I$; hence $\operatorname{cf}\left(f_{\alpha}\right)$ is the constant $\mu$ modulo $I$, and we have verified that $\alpha$ is good.

Lemma 7. Let $\alpha<\tau$ be such that $f_{\alpha}$ is the l.u.b. of $\left\langle f_{\beta} \mid \beta<\alpha\right\rangle$. Let $\mu=\operatorname{cf}(\alpha)$. Then

$$
\{i<\kappa \mid \operatorname{cf}(f(i))>\mu\} \in I .
$$

Proof of Lemma 7. If the statement of the lemma fails, then the set

$$
A=\{i<\kappa \mid \operatorname{cf}(f(i))>\mu\}
$$

is not in $I$ (it is positive with respect to $I$ ). Let $B$ be a cofinal set in $\alpha$ of order type $\mu$. Define $g \in \prod_{i<\kappa} \lambda_{i} / I_{<\kappa}$ by $g(i)=\sup \left\{f_{\beta}(i) \mid \beta \in B, f_{\beta}(i)<f_{\alpha}(i)\right\}$ if $i \in A$ and $g(i)=0$ otherwise. Clearly $g<_{I} f_{\alpha}$, so by $f_{\alpha}$ being the l.u.b. for $\left\langle f_{\beta} \mid \beta<\alpha\right\rangle$, we get $\beta<\alpha$ (and without loss of generality we may assume $\beta \in B$ ) such that $g<_{I} f_{\beta}$. But from the definition of $g$, for every $i \in B$ satisfying $f_{\beta}(i)<f_{\alpha}(i)$, we have $f_{\beta}(i)<g(i)$. We get a contradiction since $f_{\beta}$ is less than $g$ on a set which is positive with respect to $I$.

The next lemma can be considered to be a converse of Lemma 7 .

Lemma 8. Let $\alpha$ and $\mu$ be as in Lemma 7 where $\mu>\kappa$. Assume that for some $\delta>\kappa$ the set $B=\left\{i<\kappa \mid \operatorname{cf}\left(f_{\alpha}(i)\right)=\delta\right\}$ is not in I. Then $\mu=\delta$.

Proof of Lemma 8. The argument in the proof of Lemma 6 shows that if $I^{*}$ is an ideal generated by $\kappa$ sets and if $f_{\alpha}$ is the least upper bound of $\left\langle f_{\beta} \mid \beta<\alpha\right\rangle$ modulo $I^{*}$ such that, modulo $I^{*}, \operatorname{cf}\left(f_{\alpha}(i)\right)$ is constant, then this constant must be $\operatorname{cf}(\alpha)$. The assumptions of the lemma can be rephrased to say that if we put $I^{*}$ to be the ideal generated by $I$ and $\kappa-B$, then $I^{*}$ is a proper ideal and 
$f_{\alpha}$ is the l.u.b. for $\left\langle f_{\beta} \mid \beta<\alpha\right\rangle$ modulo $I^{*}$. Also, modulo $I^{*}$, the cofinality of $f_{\alpha}(i)$ is the constant $\delta$. Hence by the first part of the proof of Lemma 6, $\mu=\delta$.

We can now resume the proof of Lemma $5(\mathrm{I})$. The main tool is Claim 1.2 from [Sh6]. (In the sequel we shall refer to it as the main fact.) Let $\alpha$ be as in the statement of the lemma (I). In particular $\operatorname{cf}(\alpha)>\kappa^{+}$. If $\left\langle f_{\beta} \mid \beta<\alpha\right\rangle$ has no least upper bound, then by the main fact either $\alpha$ is bad (actually the $s_{i}$ 's witnessing it satisfy $\left.\left|s_{i}\right| \leq \kappa\right)$ or $\alpha$ is chaotic. So assume that there is a l.u.b. for $\left\langle f_{\beta} \mid \beta<\alpha\right\rangle$. (Hence $f_{\alpha}$ is such l.u.b.) We distinguish two cases:

Case 1. The set

$$
A=\left\{i<\kappa \mid \operatorname{cf}\left(f_{\alpha}(i)\right) \geq \lambda\right\} \in I .
$$

Recall that $\tau=\lambda^{+\kappa+1}, \operatorname{cf}(\alpha)=\mu$; hence $\mu=\lambda^{+\delta+1}$ for some $\delta<\kappa$. By Lemma 7 we know that the set

$$
B=\left\{i<\kappa \mid \operatorname{cf}\left(f_{\alpha}(i)\right)>\mu\right\}
$$

is in $I$. Consider the set

$$
C=\left\{i<\kappa \mid \lambda \leq \operatorname{cf}\left(f_{\alpha}(i)\right)<\mu\right\} .
$$

If $C \notin I$ then, since there are less than $\kappa$ cardinals between $\lambda$ and $\mu$ and $I$ is $\kappa$ complete, for some $\lambda \leq \eta<\mu$ the set

$$
\left\{i<\kappa \mid \operatorname{cf}\left(f_{\alpha}(i)\right)=\eta\right\} \notin I .
$$

(This is, by the way, the only place in the proof where we use the fact that $\tau=$ $\lambda^{+\kappa+1}$.) But this contradicts Lemma 8. So $C \in I$, but then $D=A \cup B \cup C \in I$, and, for $i \notin D, \operatorname{cf}\left(f_{\alpha}(i)\right)=\mu$. We have proved that the cofinality of $f_{\alpha}(i)$ is constant modulo $I$, which shows that $\alpha$ is good.

Case 2. Case 1 fails. For $i \notin A$ let $s_{i}$ be a cofinal subset of $f_{\alpha}(i)$ of order type $\operatorname{cf}\left(f_{\alpha}(i)\right)$, hence of cardinality less than $\lambda$. For $i \in A$ let $s_{i}$ be any subset of $f_{\alpha}(i)$ of cardinality less than $\lambda$. Let $D$ be any ultrafilter on $\kappa$ disjoint from $I$. Clearly $\prod_{i<\kappa} s_{i} / D$ is cofinal in $\prod_{i<\kappa} f_{\alpha}(i) / D$. We have shown that in this case $\alpha$ is bad.

This concludes the proof that $\alpha<\tau$ of cofinality greater than $\lambda$ is either good, bad, or chaotic. The fact that $\alpha$ can satisfy only one of these three possibilities is exactly as in the proof of the main fact. The same argument also works in the case $\operatorname{cf}(\alpha)=\lambda$, so we have verified parts (I) and (II) of the lemma.

For the proof of part (III), assume that $\alpha$ is good. Use Lemma 6 and get a cofinal subset of $\alpha, B$, and a set $A \in I$, such that for $i \notin A, \beta<\gamma$ in $B$, $f_{\beta}(i)<f_{\gamma}(i)$. The closed unbounded $C$ will be the set of limit points of the set $B$. Any such limit point $\delta$ of cofinality greater than $\kappa$ satisfies the claim of Lemma 6 , using the same $A$ and $B \cap \lambda$ for $B$. So in this case we have verified (III) of the lemma.

Now assume that $\alpha$ is bad. Let $s_{i}$ for $i<\kappa$ be sets of ordinals of cardinality less than $\lambda$ such that for some ultrafilter on $\kappa, D$ disjoint from $I, \prod_{i<\kappa} s_{i} / D$ cuts $\left\langle f_{\beta} \mid \beta<\alpha\right\rangle$ cofinally. Namely, for every $\beta<\alpha$ there is $g \in \prod_{i<\kappa} s_{i} / D$ and $\gamma(\beta)<\alpha$ such that

$$
f_{\beta}<_{D} g<_{D} f_{\gamma(\beta)} \text {. }
$$


One can easily get a closed unbounded subset $C$ of $\alpha$ such that, for $\beta<\delta$ in $C, \gamma(\beta)<\delta$. If $\beta$ is a limit point of $C$, then $\beta$ is clearly bad, as witnessed by the same sequence of sets $s_{i}$.

The last case is that $\alpha$ is chaotic. Let $g \in \prod_{i<\kappa} \lambda_{i} / I_{<\kappa}$ be the witness to the fact that $\alpha$ is chaotic. Namely, if we define for $\beta<\alpha$

$$
t_{\beta}=\left\{i<\kappa \mid f_{\beta}(i)<g(i)\right\},
$$

then the $t_{\beta}$ 's are positive modulo $I$, but they are not fixed modulo $I$. Hence for every $\beta<\alpha$ there is $\beta<\gamma(\beta)<\alpha$ such that $t_{\beta}$ and $t_{\gamma(\beta)}$ are different modulo $I$. As in the previous case, get a closed unbounded subset of $\alpha, C$, such that, for $\beta<\delta$ in $C, \gamma(\beta)<\delta$. Any limit point of $C$ will be chaotic using the same function $g$. This proves Lemma 5 .

So far we did not use the fact that the sequence $\left\langle f_{\alpha} \mid \alpha<\tau\right\rangle$ is cofinal in $\prod_{i<\kappa} \lambda_{i} / I_{<\kappa}$. This will play a major role in the next lemma.

Lemma 9. The set

$$
S=\{\alpha<\tau \mid \operatorname{cf}(\alpha)=\lambda, \alpha \text { is } \operatorname{good}\}
$$

is stationary in $\tau$.

Proof of Lemma 9. Let $C$ be a closed unbounded subset of $\tau$. We shall produce a member of $S \cap C$. Let $\sigma=\left(2^{r}\right)^{+}$and consider the structure $\mathscr{A}=\left\langle H_{\sigma}, \in\right\rangle$ where $H_{\sigma}$ is the set of all sets hereditarily of cardinality less than $\sigma$. Let $\left\langle N_{i} \mid i<\lambda\right\rangle$ be an increasing sequence of elementary substructures of $\mathscr{A}$, each of cardinality $\lambda$ such that for $i<j \quad N_{i} \in N_{j}$. We also assume that $\left\langle f_{\alpha} \mid \alpha<\tau\right\rangle$ and $C$ as well as all the members of $\kappa$ are in $N_{0}$. Let $N$ be the union of the $N_{i}$ 's. For $i<\lambda$ let $\alpha_{i}$ be $\sup \left(N_{i} \cap \tau\right)$ and let $\alpha$ be $\sup (N \cap \tau)=\sup \left\{\alpha_{i} \mid i<\lambda\right\}$. Clearly $\operatorname{cf}(\alpha)=\lambda$ (The $\alpha_{i}$ 's are increasing!) and since $C \in N$ we have $\alpha \in C$. If we show that $\alpha$ is good, we shall show that $S \cap C \neq \varnothing$.

We shall show that $\alpha$ is good by verifying the condition which is claimed by Lemma 6 to be equivalent to it. For $i<\lambda$ let $\xi_{i}$ be the function defined on $\kappa$ by $\xi_{i}(m)=\sup \left(N_{i} \cap \lambda_{m}\right)$. Note that $\xi_{i}$ is a member of $\Pi_{m<\kappa} \lambda_{i}$ and since $\xi_{i}$ is definable from $N_{i}$, we have for $i<j \quad \xi_{i} \in N_{j}$. Since $\kappa \subset N_{j}$, we get that all the values of $\xi_{i}$ are in $N_{j}$, so that $\xi_{i}(m)<\xi_{j}(m)$ for every $m \in \kappa$.

Since $\left\langle f_{\alpha} \mid \alpha<\tau\right\rangle$ are cofinal in $\prod_{i<\kappa} \lambda_{i} / I_{<\kappa}$, for every $i<\lambda$ there is $\beta_{i}$ such that $\xi_{i}<_{I} f_{\beta_{i}}$. Note that we must have $\alpha_{i} \leq \beta_{i}$ because otherwise we shall have, for some $\gamma \in N_{i} \cap \tau, \beta_{i} \leq \gamma$, but since all the values of $f_{\gamma}$ are in $N_{i}$ we get for all $m<\kappa$ that $f_{\gamma}(m)<\xi_{i}(m)$, so

$$
f_{\beta_{i}} \leq_{I} f_{\gamma}<_{I} \xi_{i}
$$

which is a contradiction. Also by elementarity, if $k<j$, then $\beta_{i}$ is in $N_{j}$, so we get

$$
\beta_{i}<\alpha_{j} \leq \beta_{j}
$$

so that the sequence $\left\langle\beta_{i} \mid i<\lambda\right\rangle$ is increasing and cofinal in $\alpha$. We clearly have $f_{\beta_{i}} \in N_{j}$. Again, it implies that all the values of $f_{\beta_{i}}$ are in $N_{j}$, so that, for every $m<\kappa, f_{\beta_{i}}(m)<\xi_{j}(m)$. For $i<\lambda$, let

$$
A_{i}=\left\{m<\kappa \mid f_{\beta_{i}}(m) \leq \xi_{i}(m)\right\} \text {. }
$$


By definition of $\beta_{i}, A_{i} \in I$ for all $i<\lambda$. Since $I$ is generated by $\kappa$ sets, we can find a set $A \in I$ such that the set

$$
B=\left\{i<\lambda \mid A_{i} \subseteq A\right\}
$$

is unbounded in $\lambda$. Let $B^{*}=\left\{\beta_{i} \mid i \in B\right\}$. We know that $B^{*}$ is cofinal in $\alpha$. We claim that the pair $A$ and $B$ witnesses, through Lemma 6 , the fact that $\alpha$ is good. Let $\beta_{i}<\beta_{j}$ be two members of $B^{*}$. (Of course $i<j$.) For $m \notin A$ we have

$$
f_{\beta_{i}}(m)<\xi_{j}(m)<f_{\beta_{j}}(m)
$$

(where the first inequality holds for every $m<\kappa$ and the second one holds for every $m \notin A_{j}$, but $A_{j} \subseteq A$.) The last inequality proves that $\alpha$ is good.

We are ready to define the stationary set $S$ and the sequence $\left\langle A_{\alpha} \mid \alpha \in S\right\rangle$ that will witness the truth of Theorem 4 . Let $S$ be the set $\{\alpha<\tau \mid \operatorname{cf}(\alpha)=\lambda$, $\alpha$ is good . By Lemma $9 S$ is a stationary subset of $\tau$. Each $f_{\alpha}$ is a subset of $\kappa \times \lambda^{+\kappa}$ of cardinality $\kappa$. By simple coding we can consider it to be a subset of $\lambda^{+\kappa}$ of cardinality $\kappa$. Denote the coded version of $f_{\alpha}$ by $A_{\alpha}$. We claim that $S$ and $\left\langle A_{\alpha} \mid \alpha \in S\right\rangle$ satisfy the statement of Theorem 4. This will follow from

Lemma 10. Let $\gamma<\delta<\tau$. Let $S_{\gamma}^{\delta}=\{\alpha \in S \mid \gamma<\alpha \leq \delta\}$. Then for $\alpha \in S_{\gamma}^{\delta}$ we can define $B_{\alpha} \subseteq A_{\alpha}$ and $D_{\alpha}$, a closed unbounded subset of $\alpha$, such that $\left|A_{\alpha}-B_{\alpha}\right|<\kappa$ and $D_{\alpha} \cap \gamma=\varnothing$, and for $\alpha, \beta \in S_{\gamma}^{\delta}, \alpha \neq \beta$, we have that $B_{\alpha} \times D_{\alpha}$ and $B_{\beta} \times D_{\beta}$ are mutually disjoint.

Theorem 4 follows easily from Lemma 10 , because if $T$ is a nonstationary subset of $S$, we can pick $C$, which is a closed unbounded subset of $\tau$ disjoint from $T$. If $\gamma<\delta$ are two adjacent members of $C$, we can apply Lemma 10 to the $S_{\gamma}^{\delta}$ and get $B_{\alpha}, D_{\alpha}$ for $\alpha \in S_{\gamma}^{\delta}$ as in Lemma 10. Note that every member of $T$ is in $S_{\gamma}^{\delta}$ for some two adjacent members of $C, \gamma<\delta$. Putting together the choices of $B_{\alpha}$ 's and $D_{\alpha}$ 's for the different $S_{\gamma}^{\delta}$ gives witness to the truth of Theorem 4. The main point is that if $\alpha<\beta \in T$ and $\gamma<\delta$ are two adjacent points of $C$ such that $\gamma<\beta<\delta$, then if $\alpha$ is in $S_{\gamma}^{\delta}$ the disjointness of $B_{\alpha} \times D_{\alpha}$ and $B_{\beta} \times D_{\beta}$ follows from Lemma 10. If $\alpha \notin S_{\gamma}^{\delta}$ (which is equivalent to $\alpha<\gamma)$, then $D_{\alpha}$ and $D_{\beta}$ are disjoint since $D_{\beta} \cap \gamma=\varnothing$, and so $B_{\alpha} \times D_{\alpha}$ is disjoint from $B_{\beta} \times D_{\beta}$.

Proof of Lemma 10. The proof of Lemma 10 is by induction on $\delta$. We first deal with the case that $\operatorname{cf}(\delta) \leq \lambda$ or $\operatorname{cf}(\delta)>\lambda$ and $\delta$ is not good. In this case we can find a closed unbounded subset of $\delta, C$, disjoint from $S_{\gamma}^{\delta}$. We get such a $C$ because if $\operatorname{cf}(\delta) \leq \lambda$ we can assume that every point in $C$ has cofinality $<\lambda$ (hence it is not $S$ ), and if $\delta$ is not good (hence bad or chaotic), we can use Lemma 5 to get a closed unbounded $C$ such that every point in $C$ is not good, hence not in $S$. We can also assume that the first point in $C$ is $\gamma$. Each $\alpha \in S_{\gamma}^{\delta}$ (except maybe $\delta$, if $\delta \in S$ ) belongs to a unique $S_{\rho}^{\mu}$ where $\rho<\mu<\delta$ are two adjacent points in $C$. Using the induction assumption for $\mu$, we can 
get a choice of $B_{\alpha}, D_{\alpha}$ for $\alpha \in S_{\rho}^{\mu}$ which will satisfy the requirements of our lemma. Combining the choices of $B_{\alpha}$ 's and $D_{\alpha}$ 's made by different pairs of adjacent points of $C, \rho<\mu$ gives the required choice for $S_{\gamma}^{\delta}$ by an argument similar to the argument above, deriving Theorem 4 from Lemma 10. We still have to define $B_{\delta}$ and $D_{\delta}$ if $\delta \in S$. But in this case we put $B_{\delta}=A_{\delta}$ and $D_{\delta}=C$. This choice does not conflict with the choices of $B_{\alpha}$ and $D_{\alpha}$ for $\alpha \in S_{\gamma}^{\delta}, \alpha<\delta$, because by our construction $D_{\alpha}$ is disjoint from $C=D_{\delta}^{\alpha}$.

The interesting case is the case when $\operatorname{cf}(\delta)>\lambda$ and $\delta$ is good. We use Lemma 6 and get a set $D$ cofinal in $\delta$ and a set $A$ in the ideal $I$ such that for $\rho<\mu$ in $D$ and $\nu \notin A$ we have $f_{\rho}(\nu)<f_{\mu}(\nu)$. We are assuming the first point of $D$ is above $\gamma$. Let $C$ be the closed unbounded subset of $\delta$ formed by the limit points of $D$ with the additional point $\gamma$. Every point in $S_{\gamma}^{\delta}$ is either a limit point of $C$ (hence a limit point of $D$ ) or is a member of $S_{\rho}^{\mu}$ for a unique pair $\rho<\mu$ of adjacent points of $C$. If $\rho<\mu$ are adjacent points in $C$, we use the induction assumption for $\mu$ and fix sequences $\left\langle B_{\alpha} \mid \alpha \in S_{\rho}^{\mu}\right\rangle$ and $\left\langle D_{\alpha} \mid \alpha \in S_{\rho}^{\mu}\right\rangle$ satisfying the requirements of our lemma. So we get $B_{\alpha}$ and $D_{\alpha}$ defined for every $\alpha \in S_{\gamma}^{\delta}$ which is not a limit point of $C$. We still have to define $B_{\alpha}$ and $D_{\alpha}$ for $\alpha$ which is a limit point of $C$ and belongs to $S$. (We do not have to deal with $\delta$ because $\operatorname{cf}(\delta)>\lambda$, and hence $\delta \notin S$.)

Fix an $\alpha<\delta$ such that $\alpha \in S$ and $\alpha$ is a limit point of $C$. For $\beta<\alpha$ let $E_{\beta}=\left\{\nu<\kappa \mid \nu \in A\right.$ or $\left.f_{\beta}(\nu) \geq f_{\alpha}(\nu)\right\}$. Note that $E_{\beta}^{\alpha} \in I$. By definition of $D$ and $A$ the sequence of sets $\left\langle E_{\beta}^{\alpha} \mid \beta \in D \cap \alpha\right\rangle$ is an increasing sequence of subsets of $\kappa . D$ is cofinal in $\alpha$ and $\operatorname{cf}(\alpha)=\lambda>\kappa$; hence this sequence is eventually constant. Let $F_{\alpha}$ be subset of $\kappa$ such that, for large enough $\beta \in D \cap \alpha$, $E_{\beta}^{\alpha}=F_{\alpha}$. Of course we have $F_{\alpha} \in I$. Let $\eta(\alpha)$ be the first member of $D$ above $\alpha$. Note that $\eta(\alpha)$ is smaller than the first member of $C$ above $\alpha$. We have that $f_{\alpha}<_{I} f_{\eta(\alpha)}$; hence the set

$$
H_{\alpha}=\left\{\nu<\kappa \mid \nu \in F_{\alpha} \text { or } f_{\alpha}(\nu) \geq f_{\eta(\alpha)}(\nu)\right\}
$$

is in $I . \quad B_{\alpha}$ is the set of members of $A_{\alpha}$ coding the pairs $\left\langle\nu, f_{\alpha}(\nu)\right\rangle$ for $\nu \notin H_{\alpha}$. Since $I$ is the ideal $I_{<\kappa}$ and $H_{\alpha} \in I$, we have $\left|A_{\alpha}-B_{\alpha}\right|<\kappa$. We also define $D_{\alpha}=C \cap \alpha$. This completes the definition of $B_{\alpha}$ and $D_{\alpha}$ for $\alpha \in S_{\gamma}^{\delta}$. We claim that it satisfies the conclusion of our lemma. The only nontrivial fact is the mutual disjointness of $B_{\alpha} \times D_{\alpha}$ and $B_{\beta} \times D_{\beta}$ for $\alpha<\beta$, both in $S_{\gamma}^{\delta}$. If both $\alpha$ and $\beta$ are not limit points of $C$, then the disjointness is argued as above. If one of them, say $\alpha$, is a limit point of $C$ where the other one is not, then note that $D_{\beta}$ is disjoint from $C$, hence disjoint from $D_{\alpha}$. The last (and interesting) case is when both $\alpha$ and $\beta$ are limit points of $C$. In this case we claim that $B_{\alpha}$ and $B_{\beta}$ are disjoint. In view of the definition of $B_{\alpha}$ and $B_{\beta}$ the claim amounts to the fact that if $\nu \in \kappa$ and $\nu \notin H_{\alpha} \cup H_{\beta}$, then $f_{\alpha}(\nu) \neq f_{\beta}(\nu)$. Let $\eta=\eta(\alpha)$ as above. We have $\eta<\beta$. Let $\xi$ be a member of $D, \eta<\xi<\beta$, such that $E_{\xi}^{\beta}=F_{\beta}$. If $\nu<\kappa$ is not in $H_{\alpha} \cup H_{\beta}$, we have 
$\nu \notin A$ and $\nu \notin E_{\xi}^{\beta}$. Hence (using $\eta, \xi \in D$ )

$$
f_{\alpha}(\nu)<f_{\eta}(\nu)<f_{\xi}(\nu)<f_{\beta}(\nu) .
$$

Therefore, $f_{\alpha}(\nu) \neq f_{\beta}(\nu)$ and $B_{\alpha}$ is disjoint from $B_{\beta}$.

This completes the proof of Theorem 4.

\section{SUFFICIENT CONDITIONS FOR COMPACTNESS}

In this section we formulate a sufficient condition for a cardinal $\kappa$ to be compact. The general framework is an abstract notion of freeness as introduced by Shelah ([Sh1]; see also [Sh4]). We are given a set $H$, and we have a family of pairs of subsets of $H, \mathscr{F}$. Intuitively, $(A, B) \in \mathscr{F}$ means " $A$ is free over $B$ ". As an example, if $H$ is an Abelian group, then $(A, B) \in \mathscr{F}$ if $G(A \cup B) / G(B)$ is free where $G(C)$, for $C \subseteq H$, is the subgroup of $H$ generated by $C$. Following this example we say in the abstract setting that $A / B$ is free if $(A, B) \in \mathscr{F}$.

There are natural requirements one can impose on our notion of freeness. We reproduce those axioms from [Sh4] we need. (Note that the set of axioms has one cardinal parameter $\chi$.) We follow the notation for the individual axioms used in [Sh4]; hence the numbering of the axioms is not consecutive. (We do not need some of the axioms of [Sh4], so we skip them.) Also we use for instance Axiom $I^{* *}$, which is a version of Axiom $I$ in [Sh4]. For stating the axioms we need

Definition. I. We say that for the $\chi$ majority of subsets of $H$ a certain property holds if there exists an algebra on $H$ with $\chi$ operations such that for every subalgebra the property holds.

II. A sequence of sets $\left\langle A_{\alpha} \mid \alpha<\lambda\right\rangle$ is said to be continuous if, for limit $\alpha<\lambda$, $A_{\alpha}=\bigcup_{\beta<\alpha} A_{\beta}$.

Axiom $\mathrm{I}^{* *}$. If $A / B$ is free, $A^{*} \subseteq A$, then $A^{*} / B$ is free.

Axiom II. (a) $A / B$ is free iff $A \cup B / B$ is free.

(b) $A / A$ is free.

Axiom III. If $A / B$ is free, $C \subseteq B$, and $B / C$ is free, then $A / C$ is free.

Axiom IV. If $A_{i}(i<\lambda)$ is increasing and continuous, $A_{0} / B$ is free, and, for all $i<\lambda, A_{i+1} / A_{i} \cup B$ is free, then $\cup A_{i} / B$ is free.

Axiom VI. If $A / B \cup C$ is free, then, for the $\chi$ majority of $X \subseteq A \cup B, A \cap$ $X /(B \cap X) \cup C$ is free.

Axiom VII. If $A / B$ is free, then, for the $\chi$ majority of $X \subseteq A, A / X \cup B$ is free.

(Note that for the Abelian group example Axioms VI and VII hold for $\chi=\aleph_{0}$.)

Axiom A. Suppose $A / B \cup C$ is not free. If $\delta=|A|+\chi$, then, for the $\delta$ majority of $X \subseteq B, A / X \cup C$ is not free.

(For instance, in the Abelian group case if $A / B \cup C$ is not free, it is enough to have an $X \subseteq B$ such that $G(A) \cap G(B \cup C)$ is included in $G(X \cup C)$.) 
Definition. Given a notion of freeness $\mathscr{F}, A / B$ is $\lambda$ free with respect to $\mathscr{F}$ if for every subset $C$ of $A$ of cardinality $<\lambda, C, C / B$ is free.

Shelah in [Sh1] proved that if $\lambda$ is singular and our notion of freeness satisfies Axioms II, III, IV, VI, VII (for $\chi<\lambda$ ), and $A / B$ is $\lambda$ free, then $A / B$ is $\lambda^{+}$ free. (See subsequent work in [Bd] and [Ho].) Note that under Axiom $I^{* *}$ if $A / B$ is free, then it is $\lambda$ free for all $\lambda$. Also if $\lambda$ is a regular cardinal $>\chi$ and $A=\bigcup_{i<\lambda} A_{i}$ with $\left|A_{i}\right|<\lambda$ and $A_{i} / B$ free, then $A / B$ is free if and only if $S=\left\{i<\lambda|A| A_{i} \cup B\right.$ is not $\lambda$ free $\}$ is nonstationary in $\lambda$. The "if" direction of the last claim follows from Axiom IV since we have a closed unbounded subset of $\lambda, C$, such that for $\alpha \in C$ and $\beta>\alpha, A_{\beta} / A_{\alpha} \cup B$ is free. So we can write $\bigcup_{\alpha<\lambda} A_{\alpha}$ as a continuous increasing union as required by Axiom IV. The other direction follows from Axiom VI (where in the axiom we take $B=\varnothing, C=B$ ). We get the algebra witnessing Axiom VI. The set $\left\{\alpha<\lambda \mid A_{\alpha}\right.$ is a subalgebra $\}$ is a closed unbounded subset of $\lambda$ disjoint from $S$.

We shall now formulate several reflection principles which will help us get compactness results.

$\left(\Delta_{\kappa, \lambda}\right)$ For every $\aleph_{0} \leq \mu<\kappa, S \subseteq \lambda$ such that $S$ is stationary in $\lambda$, and such that $\delta \in S$ implies $\operatorname{cf}(\delta)<\kappa$, and for every algebra $A$ on $\lambda$ with $\mu$ operations, there is a subalgebra $A^{\prime}$ whose order type (as a subset of $\lambda$ ) is a regular cardinal $\eta, \eta<\kappa$, and such that $S \cap A^{\prime}$ is stationary in $\sup \left(A^{\prime}\right)$.

$\left(\Delta_{\kappa}\right)$ For every regular $\lambda \geq \kappa, \Delta_{\kappa, \lambda}$ holds.

The main theorem of this section is

Theorem 1. Let $\mathscr{F}$ be a notion of freeness satisfying Axioms I**, II, III, IV, VI, VII, and A for some $\chi<\kappa$. Let $\lambda^{*}$ be a cardinal $\geq \kappa$ such that for all regular $\lambda, \lambda \leq \lambda^{*}$, we have $\Delta_{\kappa, \lambda}$; then every pair $(A, B)$ which is $\kappa$ free with $|A| \leq \lambda^{*}$ is free.

Proof. We start by proving

Lemma 2. Let $\mathscr{F}$ be as in the hypothesis of the theorem. Assume $C / B \cup D$ is not free, while $D / B$ free. Then there exists $E \subseteq D,|E| \leq|C|+\chi$, such that, for all $E \subseteq Y \subseteq D, C / B \cup Y$ is not free.

Thus $E$ can be considered to be the "evidence" of the nonfreeness of $C / B \cup$ $D$, in the sense that once a subset $Y$ of $D$ contains this evidence then $C / B \cup Y$ is not free.

Proof. By Axiom A, if we put $\delta=|C|+\chi$, for the $\delta$ majority of $X \subseteq D$ $C / B \cup X$ is not free. Also by Axiom VI for the $\chi$ majority of $X \subseteq D \quad D / X \cup B$ is free. Hence we can find $E$ which is closed under the operations of both algebras where $|E| \leq \delta$. Hence $D / E \cup B$ is free, and $C / B \cup E$ is not free. $E$ is the required set because if $E \subseteq Y \subseteq D$, then by Axiom $I^{* *} Y / E \cup B$ is free. Hence $Y \cup B / E \cup B$ is free. If we had $C / Y \cup B$ free, then by Axiom III $C / E \cup B$ is free, contradiction.

For future applications we need a weaker version of the reflection principle.

$\left(\Delta_{\kappa, \lambda}^{-}\right)$For every $\aleph_{0} \leq \mu<\kappa, S \subseteq \lambda$ such that $S$ is stationary in $\lambda$, and such that $\delta \in S$ implies $\operatorname{cf}(\delta)<\kappa$, and for every algebra $A$ on $\lambda$ with $\mu$ operations and for every sequence of sets $\left\langle D_{\delta} \mid \delta \in S\right\rangle$ such that, for $\delta \in S$, 
$D_{\delta} \subseteq \delta,\left|D_{\delta}\right| \leq \mu$, there is a subalgebra $A^{\prime}$ whose cardinality is a regular cardinal $\eta, \eta<\kappa$, and such that $A^{\prime}$ can be written as a continuous increasing union $A^{\prime}=\bigcup_{i<\eta} A_{i}$, where the cardinality of each $A_{i}$ is less than $\eta$, each $A_{i}$ is bounded in $\sup \left(A^{\prime}\right)$, and the set $T=\left\{i<\eta \mid \delta=\sup \left(A_{i}\right)\right.$ implies $\delta \in$ $\left.S \cap A^{\prime}, D_{\delta} \subseteq A_{i}\right\}$ is stationary in $\eta$.

It follows easily that if $A^{\prime}$ is the subalgebra of $A$ witnessing $\Delta_{\kappa \lambda}^{-}$for the stationary set $S$, then $S \cap A^{\prime}$ is stationary in $\sup \left(A^{\prime}\right)$; hence it follows that for every $S$ a stationary subset of $\lambda$ whose points have cofinality less than $\kappa$, there exists $\beta<\lambda$ such that $S \cap \beta$ is stationary in $\beta$ ("S reflects").

Claim. $\Delta_{\kappa, \lambda}$ implies $\Delta_{\kappa, \lambda}^{-}$.

Proof. If $A, S$, and $\left\langle D_{\delta} \mid \delta \in S\right\rangle$ are as in the statement of $\Delta_{\kappa, \lambda}^{-}$, we can assume without loss of generality that for every subalgebra of $A, B$, if $\delta \in B \cap S$ then $D_{\delta} \subseteq B$. Let $A^{\prime}$ be a subalgebra of $A$ witnessing the truth of $\Delta_{\kappa, \lambda}$. The order type of $A^{\prime}$ is $\eta$, which is a regular cardinal. So let $f$ be an order-preserving function from $\eta$ onto $A^{\prime}$. The set

$$
Q=\left\{i<\eta \mid f^{\prime \prime} i \text { is a subalgebra }\right\}
$$

is a closed unbounded subset of $\eta$. Similarly using the fact that $S \cap A^{\prime}$ is stationary in $\sup \left(A^{\prime}\right)$, we get that

$$
R=\left\{i<\eta \mid \sup \left(f^{\prime \prime} i\right) \in S \cap A^{\prime}\right\}
$$

is stationary in $\sup \left(A^{\prime}\right)$. For $i \in R, \sup \left(f^{\prime \prime}\right)=f(i)$, but, for $i \in R, D_{f(i)} \subseteq$ $f(i) \cap A^{\prime}$; hence $D_{f(i)} \subseteq f^{\prime \prime} i$. Let $g$ be an increasing enumeration of the closed unbounded set $Q$. For $i<\eta$ let $A_{i}=f^{\prime \prime} g(i) . A^{\prime}=\bigcup_{i<\eta} A_{i}$ is a representation of $A^{\prime}$ as a continuous union as required by $\Delta_{\kappa, \lambda}^{-}$. The set $T$ in the statement of $\Delta_{\kappa, \lambda}^{-}$contains $g^{-1}(R)$, and hence it is stationary in $\eta$.

Theorem 1 will now follow immediately from

Theorem 3. Let $\mathscr{F}$ be a notion of freeness satisfying Axioms I**, II, III, IV, VI, VII, and A for some $\chi<\kappa$. Let $\lambda^{*}$ be a cardinal $\geq \kappa$ such that for all regular $\lambda, \lambda \leq \lambda^{*}$, we have $\Delta_{\kappa, \lambda}^{-}$. Then every pair $(A, B)$ which is $\kappa$ free with $|A| \leq \lambda^{*}$ is free.

Proof of Theorem 3. Assume $A / B$ is $\kappa$ free but not free with $|A|$ minimal. We distinguish two cases:

Case I. $|A|=\lambda>\kappa$.

In order to simplify notation we assume that $A=\lambda$. Note that by minimality of $|A|$, if $|A|=\lambda$, then $A / B$ is $\lambda$ free. By Shelah singular cardinal compactness, $\lambda$ is regular. Write $A=\bigcup_{i<\lambda} A_{i}$ as an increasing continuous union where $|A|<\lambda$. (Note that $A_{i} / B$ is free by assumption.) By a remark we made above, $\left\{i \mid i<\lambda, A / A_{i} \cup B\right.$ is not $\lambda$ free $\}$ is stationary in $\lambda$; hence there is $C_{i} \subseteq A,\left|C_{i}\right|<\lambda$, and $C_{i} / A_{i} \cup B$ is not free. Pick $C_{i}$ to be of minimal cardinality. By minimality of $|A|$ we know that $C_{i} / A_{i} \cup B$ cannot be $\kappa$ free, and hence, by minimality of $C_{i},\left|C_{i}\right|<\kappa$. By Lemma 2 , since $C_{i} / A_{i} \cup B$ is 
not free, whereas $A_{i} / B$ is free, we can find $D_{i} \subseteq A_{i},\left|D_{i}\right| \leq \chi+\left|C_{i}\right|$, and if $D_{i} \subseteq Y \subseteq A_{i}$ then $C_{i} / Y \cup B$ is not free.

We claim that without loss of generality we can assume that for $\delta \in S$, the cofinality of $\delta$ is less than $\kappa$; otherwise $\{\delta \mid \delta \in S \operatorname{cf}(\delta)<\kappa\}$ is nonstationary. Note that $\left|D_{i}\right|<\kappa$ and that, for most $i \in S, \operatorname{cf}(i) \geq \kappa$; hence for most members of $S, i$, we can find $h(i)<\kappa$ such that $D_{i} \subseteq A_{h(i)}$. By Fodor's lemma there is a stationary subset of $S, S^{*}$, such that $h$ is constant on it, say $h(i)=j_{0}$ for $i \in S^{*}$. Let $j \geq j_{0}$. We claim that $A / A_{j} \cup B$ is not $\lambda$ free. Pick $i \in S^{*}, j<i, D_{i} \subseteq A_{h(i)}=A_{j_{0}} \subseteq A_{j} \subseteq A_{i}$. By a property of $D_{i}$, $C_{i} / A_{j} \cup B$ is not free, and hence $A / A_{j} \cup B$ is not $\lambda$ free. By definition of $S$ we get that if $j \geq j_{0}$ then $j \in S$. Hence $S$ contains a tail of $\lambda$; in particular, stationary many points in $S$ have cofinality less than $\kappa$, a contradiction. So we can assume that we have a stationary $S$ such that, for $i \in S, \operatorname{cf}(i)<\kappa$ and $A / A_{i} \cup B$ is not $\kappa$ free. Without loss of generality we can assume that, for $i \in S,\left|D_{i}\right|,\left|C_{i}\right| \leq \mu<\kappa$ for some fixed $\mu$.

Using the fact that $\left|D_{i}\right|,\left|C_{i}\right| \leq \mu$ we can easily define an algebra with $\mu$ operations such that if $X$ is a subalgebra of it then

(a) $\mu+1 \subseteq X$

(b) if $i \in X$ then $\sup \left(A_{i}\right) \in X$;

(c) if $\alpha \in X$ then the minimal $i$ such that $\alpha \in A_{i}$ is in $X$;

(d) if $i \in X \cap S$ then $D_{i} \cup C_{i} \subseteq X$.

Now we use $\Delta_{\kappa, \lambda}^{-}$for our algebra and the stationary set $S$. We get a subalgebra $A^{\prime}$ such that the cardinality of $A^{\prime}$ is a regular cardinal $\eta<\kappa$ (note that by $\left.\mu+1 \subseteq X_{0}, \mu<\eta\right)$ and such that $A^{\prime}$ can be written as a continuous increasing union $\bigcup_{j<\eta} X_{j}$ where $X_{j}$ (for $j<\eta$ ) is a subalgebra of cardinality less than $\eta$, and the set

$$
T=\left\{j<\eta \mid \sup \left(X_{j}\right) \in S \cap A^{\prime}, D_{\sup \left(X_{j}\right)} \subseteq X_{j}\right\}
$$

is stationary in $\eta$. Fix $j<\eta$ and let $\delta=\sup \left(X_{j}\right)$. By the properties of the algebra $A$ (recall that $X_{j}$ is a subalgebra), we know that $X_{j} \subseteq A_{\delta} \cdot A_{\delta} / B$ is free, and hence $X_{j} / B$ is free (by Axiom $\mathrm{I}^{* *}$ ). It follows that $A^{\prime} / B$ is free iff the set

$$
T^{*}=\left\{j<\eta \mid A^{\prime} / X_{j} \cup B \text { is not } \eta \text { free }\right\}
$$

is nonstationary in $\eta$. But if $j \in T$ we have that $\delta \in S \cap A^{\prime}$ and that $D_{\delta} \subseteq X_{j}$. Also $C_{\delta} \subseteq A^{\prime}$. By definition of $D_{\delta}$ we get $C_{\delta} / X_{j} \cup B$ is not free, so $A^{\prime} / X_{j} \cup B$ is not $\eta$ free, so we have proved $T \subseteq T^{*}$. Since $T$ is stationary in $\eta$, we conclude that $A^{\prime} / B$ is not free, so $A / B$ is not $\kappa$ free. We get a contradiction to our assumption that $A / B$ is $\kappa$ free but not free.

Case II. $|A|=\kappa$.

Of course in this case (in view of Shelah singular compactness) $\kappa$ is regular.

We follow the proof of Case I and define $A_{i}, S, C_{i}$, and $D_{i}$ as in Case I. As in Case I we assume without loss of generality that $A=\kappa$. The only difference is that now we cannot assume without loss of generality that, for some $\mu<\kappa$ and for $i \in S,\left|D_{i}\right|,\left|C_{i}\right| \leq \mu$. Note that if, for a stationary subset $S^{*} \subseteq S$ and 
$i \in S^{*},\left|C_{i}\right|<i$, then $\left|D_{i}\right|<i$ and by Fodör's lemma we can find $S^{* *} \subseteq S^{*}$, $S^{* *}$ stationary, and $\left|C_{i}\right|$ for $i \in S^{* *}$ constant, and we can conclude the proof as in Case I, so without loss of generality we assume that, for $i \in S, i \leq\left|C_{i}\right|$. Since $\left|C_{i}\right|<\kappa$, we get that $\kappa$ is a limit, hence inaccessible, cardinal. Hence we can assume without loss of generality that, for $i \in S, A_{i}=\left|A_{i}\right|=i$. Using Shelah singular compactness again, and the minimality of $\left|C_{i}\right|,\left|C_{i}\right|$ is regular. We claim

Claim. For $i \in S$, if $\chi<i$ then $\left|C_{i}\right|=i$.

Proof. By Axiom A, $C_{i} \cup A_{i} / B$ is free since $\left|C_{i} \cup A_{i}\right|<\kappa$. (Recall that $A / B$ is $\kappa$ free.) By Axiom VII, for the $\chi$ majority of $X \subseteq A_{i} \cup C_{i}, C_{i} \cup A_{i} / X \cup B$ is free.

Let $A_{i}^{*}$ be the closure of $A_{i}$ under the relevant $\chi$ operations; hence $C_{i} \cup$ $A_{i} / A_{i}^{*} \cup B$ is free. If we had $A_{i}^{*} \cup B / A_{i} \cup B$ free, then $C_{i} \cup A_{i} / A_{i} \cup B_{i}$ is free, which is a contradiction to the definition of $C_{i}$; hence $A_{i}^{*} \cup B / A_{i} \cup B$ is not free. But for $\chi<i,\left|A_{i}^{*}\right|=i$, and hence, by minimality of $\left|C_{i}\right|,\left|C_{i}\right| \leq\left|A_{i}^{*}\right| \leq i$.

It follows from the claim that if $i \in S$ then $i$ is regular. By $\Delta_{\kappa, \kappa}^{-}$we get that the set $T=\{\beta \mid S \cap \beta$ is stationary in $\beta\}$ is a stationary subset of $\kappa$. (Note that if $\beta \in T$ then $\beta$ is regular.)

By $T$ being stationary, we can find $\beta \in T$ such that if $i<\beta$ then $C_{i} \subseteq \beta$. For such a $\beta,\left|A_{\beta}\right|=\beta$ and as in Case I we can show (using $S \cap \beta$ stationary in $\beta$ ) that $A_{\beta} / B$ is not free. Hence we get a contradiction to $A / B$ being $\kappa$ free but not free. This completes the proof of Theorem 3, which was needed to finish the proof of Theorem 1.

For future applications we need an equivalent form of $\Delta_{\kappa, \lambda}$ :

Lemma 4. Assume $\Delta_{\kappa, \lambda}$ holds. Then for every algebra $\mathscr{A}=\left\langle A, f_{0}, f_{1}, \ldots\right\rangle$ with less than $\kappa$ many operations such that $\lambda \subseteq A$ and every stationary subset of $S \subseteq \lambda$ such that, for $\alpha \in S, \operatorname{cf}(\alpha)<\kappa$ there is a subalgebra of $\mathscr{A}, \mathscr{B}$, of cardinality less than $\kappa$ such that $\mathscr{B} \cap \lambda$ has an order type which is a regular cardinal and $S \cap \mathscr{B}$ is stationary in $\sup (\mathscr{B} \cap \lambda)$.

Proof. Without loss of generality assume that the operations in $\mathscr{A}$ are closed under composition. Let $\mathscr{A}\left\lceil\lambda=\left\langle\lambda, f_{0}\left\lceil\lambda, f_{1}\lceil\lambda, \ldots\rangle\right.\right.\right.$ (where $f_{\alpha}\lceil\lambda$ means that if $f_{\alpha}\left(\rho_{1}, \ldots, \rho_{k}\right) \notin \lambda, \rho_{1}, \ldots, \rho_{k}<\eta$, then $\left(f\lceil\lambda)\left(\rho_{1}, \ldots, \rho_{k}\right)=0\right)$. Use $\Delta_{\kappa, \lambda}$ for $\mathscr{A}\lceil\lambda$ and $S$, and get $\mathscr{C}$ a subalgebra of $\mathscr{A} \mid \eta$ of cardinality $<\kappa$ such that the order type of $\mathscr{C}$ is a regular cardinal and $S \cap \mathscr{C}$ is stationary in $\sup (\mathscr{C})$. Let $\mathscr{B}$ be the closure of $\mathscr{C}$ under the operations of $\mathscr{A} ; \mathscr{B}$ is the required subalgebra of $\mathscr{A}$. (Note that $\mathscr{B} \cap \lambda=\mathscr{C}$ because the operations of $\mathscr{A}$ were assumed to be closed under composition.)

\section{3. $\aleph_{\omega^{2}+1}$ CAN BE COMPACT}

Definition. A cardinal $\kappa$ is compact if, for every notion of freeness satisfying Axioms I**, II, III, IV, VI, VII, and A, $\kappa$ free implies $\kappa^{+}$free.

The main theorem of this section is 
Theorem 1. Assuming the consistency of infinitely many supercompact cardinals, there is a model of $\mathrm{ZFC}+\mathrm{G}$.C. H. in which $\aleph_{\omega^{2}+1}$ is compact. (The model will be obtained by getting a model of $\Delta_{\aleph_{\omega^{2}}, \aleph_{\omega^{2}+1}}$.)

In our ground model we fix a sequence $\left\langle\kappa_{n} \mid n<\omega\right\rangle$ of supercompact cardinals. Using the construction of Laver in [La] we can assume that our supercompacts satisfy the following assumption:

(*) If $P$ is a $\kappa_{n}$ directed closed forcing notion (i.e., every directed subset of $P$ of cardinality $<\kappa_{n}$ has a lower bound), then $V^{P} \vDash \kappa_{n}$ is supercompact. By looking at the proof of the consistency of $(*)$ in [La] one can easily verify that we can get $(*)$ to hold together with $2^{\kappa_{n}}=\kappa_{n}^{+}($for $n<\omega)$ or even $2^{\kappa_{n}^{+k}}=\kappa_{n}^{+k+1}$ for $k<\omega$. Also since the truth of $(*)$ is not changed by a $\kappa_{\omega}^{+}$directed closed forcing, we can assume without loss of generality that, for $\lambda \geq \kappa_{\omega}, 2^{\lambda}=\lambda^{+}$. Also we can assume that G.C.H. holds below $\aleph_{\omega}$.

For a pair of regular cardinals $\lambda, \mu, \lambda<\mu$, denote by $\operatorname{Col}(\lambda,<\mu)$ the Levy collapse of all cardinals less than $\mu$ to $\lambda$. (Namely, $\operatorname{Col}(\lambda,<\mu)$ is the set of all partial functions of cardinality $<\lambda$ from $\lambda \times \mu$ into $\mu$ where $f(\delta, \alpha) \in \alpha$ for $\alpha \in \mu-\lambda$ and $\alpha$ a cardinal, partially ordered by inverse inclusion.) Recall that $\operatorname{Col}(\lambda,<\mu)$ is $\lambda$ directed closed and if $\mu$ is strongly inaccessible, it satisfies the $\mu$ chain condition. Denote by $S_{n}=\prod_{m \geq n} \operatorname{Col}\left(\kappa_{m}^{+2},<\kappa_{m+1}\right)$. Note that $S_{n}$ is $\kappa_{n}$ directed (actually even $\kappa_{n}^{+2}$ directed closed); hence $V^{S_{n}} \vDash \kappa_{n}$ is supercompact. Also standard arguments show that in $V^{S_{n}} \kappa_{\omega}=\sup _{m<\omega} \kappa_{m}$ is $\kappa_{n}^{+\omega}$. Let $\dot{F}_{n}$ be a term denoting in $V^{S_{n}}$ a normal ultrafilter on $P_{\kappa_{n}}\left(\kappa_{\omega}^{+}\right)$. (It exists since $\kappa_{n}$ is supercompact in $V^{S_{n}}$; see [K-M] for definition of normal ultrafilters, etc.) $\dot{F}_{n}$ has a natural projection to a normal ultrafilter on $\kappa_{n}$ (namely, $A \in U_{n} \leftrightarrow\left\{P \mid P \cap \kappa_{n} \in A, P \in P_{\kappa_{n}}\left(\kappa_{\omega}^{+}\right)\right\} \in F_{n}$ ). Recall that we assumed that $2^{\kappa_{n}}=\kappa_{n}^{+}$, and that forcing with $S_{n}$ does not introduce new sets which are hereditarily of cardinal $\leq \kappa_{n}^{+}$; hence $U_{n} \in V$. So we get a normal ultrafilter in $V, U_{n}$, such that some condition in $S_{n}$ forces it to be the projection of some normal ultrafilter on $P_{\kappa_{n}}\left(\kappa_{\omega}^{+}\right)$. Since $S_{n}$ is a homogeneous forcing notion, we get that the empty condition in $S_{n}$ (hence any condition in $S_{n}$ ) forces that $U_{n}$ is the projection of some normal ultrafilter on $P_{\kappa_{n}}\left(\kappa_{\omega}^{+}\right)$.

Let $M_{n}$ be the transitive collapse of the ultrapower $V^{\kappa_{n}} / U_{n} . j: V \rightarrow M_{n}$ will be the corresponding elementary embedding. Members of $M_{n}$ are of the form $[f]_{U_{n}}$, which is the equivalence class of the function $f \in V^{\kappa_{n}} \bmod -$ ulo $U_{n}$. Consider the forcing notion $T=\operatorname{Col}^{M_{n}}\left(\kappa_{n}^{+\omega+2},<j_{n}\left(\kappa_{n}\right)\right.$ ) (where $\mathrm{Col}^{M_{n}} \ldots$ means the collapse forcing notion defined in $M_{n}$ ). Recall that $M_{n}$ has the property that every sequence of its members of length $\kappa_{n}$ is in $M_{n}$, that $M_{n} \vDash j\left(\kappa_{n}\right)$ is inaccessible, and $M_{n} \vDash T$ satisfies the $j_{n}\left(\kappa_{n}\right)$ chain condition. Therefore $M \vDash$ there are exactly $j_{n}\left(\kappa_{n}\right)$ dense subsets of $T$. On the other hand, $V \vDash\left|j_{n}\left(\kappa_{n}\right)\right|=\kappa_{n}^{+}$; hence we can enumerate (in $V$ ) the dense subsets of 
$T$ which lie in $M_{n}$ in a sequence of length $\kappa_{n}^{+}$. Every initial segment of this sequence is in $M_{n}$, and also $M_{n} \vDash T$ is $\kappa_{n}^{+}$closed; hence one can easily define (in $V$ ) a $T$ generic filter over $M_{n}$ by inductively meeting each dense set in $M_{n}$. Fix such a generic filter $G_{n}$.

We are at last ready to define our forcing notion $\mathscr{P}$, such that in $V^{\mathscr{P}} \vDash \Delta_{\kappa_{\omega}, \kappa_{\omega}^{+}}$ and $V^{\mathscr{P}} \vDash \kappa_{\omega}=\aleph_{\omega^{2}}$. Also $V^{\mathscr{P}}$ will satisfy the G.C.H., so it will be the required model for proving Theorem 1. The definition of the forcing uses ideas of Foreman-Woodin [F-W], Woodin [Cu-Wo], and Gitik [Gi], modifying the original construction of [Ma], of models violating the singular cardinals hypothesis.

The idea is to use the sequence of the normal ultrafilters $\left\langle U_{n} \mid n<\omega\right\rangle$ to get a generalized Prikry sequence $\left\langle\alpha_{i} \mid i<\omega\right\rangle$ such that $\kappa_{i-1}<\alpha_{i}<\kappa_{i}$ (for simplicity put $\left.\kappa_{-1}=\omega_{1}\right)$, and then collapse all cardinals in the intervals $\left(\omega_{3}, \alpha_{0}\right),\left(\alpha_{0}^{+\omega+2}, \kappa_{0}\right),\left(\kappa_{0}^{+2}, \alpha_{1}\right),\left(\alpha_{1}^{+\omega+2}, \kappa_{1}\right)$, etc., so that $\alpha_{0}$ becomes $\aleph_{4}$, $\kappa_{1}$ becomes $\aleph_{\omega+3}, \alpha_{1}$ is $\aleph_{\omega+6}\left(\kappa_{n}\right.$ is $\left.\aleph_{\omega(n+1)+3}\right), \kappa_{\omega}$ will be $\aleph_{\omega^{2}}$.

Definition of the forcing notion. $\mathscr{P}$ : The forcing notion $\mathscr{P}$ is made up of all sequences of the form

$$
\begin{aligned}
p=\left\langle\alpha_{0}, \ldots, \alpha_{n-1},\left\langle A_{j} \mid n \leq j<\omega\right\rangle,\right. & g_{0}, \\
& g_{1}, \ldots, g_{n}, f_{0}, \ldots, f_{n-1}, \\
& \left.\left\langle F_{j} \mid n \leq j<\omega\right\rangle,\left\langle g_{j} \mid n<j<\omega\right\rangle\right\rangle
\end{aligned}
$$

such that

(a) $\kappa_{i-1}<\alpha_{i}<\kappa_{i}, \alpha_{i}$ inaccessible (recall $\kappa_{-1}=\omega_{1}$ ) for $0 \leq i \leq n$;

(b) $A_{j} \in U_{j}$ for $n \leq j<\omega$, every $\alpha \in A_{j}$ is inaccessible;

(c) $f_{i} \in \operatorname{Col}\left(\alpha_{i}^{+\omega+2},<\kappa_{i}\right)(i<n)$;

(d) $g_{i} \in \operatorname{Col}\left(\kappa_{i-1}^{++},<\alpha_{i}\right) \quad(i<n)$;

(e) $g_{j} \in \operatorname{Col}\left(\kappa_{j-1}^{++},<\kappa_{j}\right), j \geq n$, such that $g_{j} \in \operatorname{Col}\left(\kappa_{j-1}^{++},<\alpha\right)$ for $\alpha \in A_{j}$. (Note that (e) implies that $\left\langle g_{j} \mid j>n\right\rangle \in S_{n}$.)

(f) For $j \geq n, F_{j}$ is a function defined on $A_{j}$ such that, for $\alpha \in A_{i}, F_{j}(\alpha) \in$ $\operatorname{Col}\left(\alpha^{+\omega+2},<\kappa_{j}\right)$ and such that the equivalence class of $F_{j}$ as a member of the ultrapower $V^{\kappa_{j}} / U_{j}$ is in $G_{j}$.

$n$ is the length of the condition $p$. Its $\alpha$-part is $\left\langle\alpha_{0}, \ldots, \alpha_{n-1}\right\rangle$. Its $A$ part is $\left\langle A_{j} \mid j \geq n\right\rangle$; similarly the $f$-part of $p$ is $\left\langle f_{0}, \ldots, f_{n-1}\right\rangle$, and the $g$-part of $p$ is $\left\langle g_{0}, \ldots, g_{n}\right\rangle$. Its $S$-part is $\left.\left\langle g_{j} \mid j\right\rangle n\right\rangle$, and its $F$-part is $\left\langle F_{j} \mid j \geq n\right\rangle$. The lower part of $p$ is 〈the $\alpha$-part of $p$, the $f$-part, the $g$-part $\rangle$. (Note that there are at most $\kappa_{n}$ different lower parts of conditions of length $n$.) For a condition $p$ as above we put $\alpha_{i}(p)=\alpha_{i}$. Similarly we define $A_{j}(p), f_{i}(p), g_{j}(p)$, and $F_{j}(p) . p\lceil k$, the restriction of $p$ to $k(k \leq n)$, is $\left\langle\left\langle\alpha_{0}, \ldots, \alpha_{k-1}\right\rangle,\left\langle g_{0}, \ldots, g_{k}\right\rangle,\left\langle f_{0}, \ldots, f_{k-1}\right\rangle\right\rangle$. Note that the lower part of $p$ is exactly $p\lceil n$.

Intuitively $\alpha_{0}, \ldots, \alpha_{n}$ is an initial segment of the "generalized Prikry sequence". $A_{j}$ is the set of possible candidates for being $\alpha_{j} \cdot g_{i}$ and $f_{i}$ are part 
of the Levy collapse, making sure that there are no cardinals in the intervals $\left(\kappa_{i-1}^{+2}, \alpha_{i}\right),\left(\alpha_{i}^{+\omega+2}, \kappa_{i}\right)$, respectively. $F_{j}(\alpha)$ is a commitment that if $\alpha \in A_{j}$ will be picked in the future as $\alpha_{j}$, then the corresponding $f_{j}$ will have to extend $F_{j}(\alpha) . g_{j}$ for $j>n$ is a commitment about the possible value of $g_{j}$ in the future. (Note that if $g_{j} \in \operatorname{Col}\left(\kappa_{j-1}^{+2},<\kappa_{j}\right)$, then, for large enough $\alpha<\kappa_{j}$, $g_{j} \in \operatorname{Col}\left(\kappa_{j-1},<\alpha\right)$, so $g_{j}$ is implicitly also the commitment to pick $\alpha_{j}$ so large that $g_{j} \in \operatorname{Col}\left(\kappa_{j-1},<\alpha\right)$.)

These intuitive remarks should motivate the definition of the partial order on $P$, where the formal definition is

$$
\begin{aligned}
p=\left\langle\alpha_{0}, \ldots, \alpha_{n-1},\left\langle A_{j} \mid j \geq n\right\rangle, g_{0}, \ldots,\right. & g_{n}, f_{0}, \ldots, f_{n-1}, \\
& \left.\left\langle F_{j} \mid j \geq n\right\rangle,\left\langle g_{j} \mid j>n\right\rangle\right\rangle
\end{aligned}
$$

extends

$$
\begin{aligned}
p^{*}=\left\langle\alpha_{0}^{*}, \ldots, \alpha_{m-1}^{*},\left\langle A_{j}^{*} \mid j \geq m\right\rangle, g_{0}^{*}, \ldots,\right. & g_{m}^{*}, f_{0}^{*}, \ldots, f_{m-1}^{*}, \\
& \left.\left\langle F_{j}^{*} \mid j \geq m\right\rangle,\left\langle g_{j}^{*} \mid j>m\right\rangle\right\rangle
\end{aligned}
$$

$\left(p \leq p^{*}\right)$ if

(a) $m \leq n$ and for $i<m, \alpha_{i}=\alpha_{i}^{*}$, and $f_{i}$ extends $f_{i}^{*}$ as a member of $\operatorname{Col}\left(\alpha_{i}^{+\omega+2},<\kappa_{i}\right)$;

(b) for $i<\omega, g_{i}$ extends $g_{i}^{*}$;

(c) for $n \leq j, A_{j} \subseteq A_{j}^{*}$ and, for all $\alpha \in A_{j}, F_{j}(\alpha)$ extends $F_{j}^{*}(\alpha)$ (as a member of $\left.\operatorname{Col}\left(\alpha^{+\omega+2},<\kappa_{j}\right)\right)$;

(d) for $m \leq i<n, \alpha_{i} \in A_{i}^{*}$ and $f_{i} \supseteq F_{i}^{*}\left(\alpha_{i}\right)$.

$p$ is a length-preserving extension of $p^{*}$ if $n=m . \quad p$ is a $k$-lengthpreserving extension (for $k \leq m$ ) if $p$ is a length-preserving extension of $p^{*}$ and $p\left\lceil k=p^{*}\left\lceil k\right.\right.$. We shall denote it by $p \leq_{k} p^{*}$. We say that $p$ is a trivial extension of $p^{*}$ if they have the same lower part. (It is the same as being $m$-length-preserving.) Being a $k$-length-preserving extension means that we did not change the information $p^{*}$ was giving below $\alpha_{k}$.

Lemma 2. Let $\left\langle p_{\delta}\right| \delta\langle\eta\rangle$ be $a \leq_{k}$ decreasing sequence all of them of length $n$, where $\eta \leq \alpha_{k}\left(p_{0}\right)$ if $k<n$ and $\eta<\kappa_{n}$ if $k=n$. Then the sequence has $a \leq_{k}$ lower bound.

Proof. We define $q$, which is going to be the $\leq_{k}$ lower bound of the sequence, by making the $\alpha$-part of $q$ equal to the common $\alpha$-part of the conditions in the sequence. For $i<n, f_{i}(q)=\bigcup_{\delta<\eta} f_{i}\left(p_{\delta}\right)$. (For $i<k$ we get $f_{i}(q)=$ $f_{i}\left(p_{0}\right)$, and for $i \geq k \quad f_{i}(q) \in \operatorname{Col}\left(\alpha_{i}^{+\omega+2},<\kappa_{i}\right)$ by the $\operatorname{Col}\left(\alpha_{i}^{+\omega+2},<\kappa_{i}\right)$ being $\alpha_{i}^{+}$closed.) Similarly we put $g_{j}(q)=\bigcup_{\delta<\eta} g_{j}\left(p_{\delta}\right)$. For $j \geq n, A_{j}(q)=$ $\bigcap_{\delta<\eta} A_{j}\left(p_{\delta}\right)-\eta$. Here we use $\eta<\kappa_{n}$ to get that $A_{j}(q) \in U_{j}$. For $j \geq n$ and $\alpha \in A_{j}(q)$ we define $F_{j}(q)(\alpha)=\bigcup_{\eta<\delta} F_{j}\left(p_{\delta}\right)(\alpha) . F_{j}(q)(\alpha)$ is easily seen to be in $\operatorname{Col}\left(\alpha^{+\omega+2},<\kappa_{j}\right)$. We have only to verify that the equivalence class of $F_{j}(q)$ is in $G_{j}$. But $M_{j}$ is closed under sequences of length $\eta$. Hence since $G_{j}$ is a generic filter for a forcing notion considered to be $\eta$ closed by 
$M_{j}$ (which means that it is $\eta$ closed in $V$ ), we get that $\bigcup_{\delta<\eta}\left[F_{j}\left(p_{\delta}\right)\right]_{U_{j}}$ is in $G_{j}$, but the last union is exactly $\left[F_{j}(q)\right]_{U_{n}}$.

The dual notion to $k$-length-preserving extension is the notion of $k$-direct extension, where we do not add any information on the collapse maps above $\alpha_{k}$, but we may change the information below $\alpha_{k}$. Formally $p$ is a $k$-direct extension of $p^{*}$ (for $k \leq n$ ) if in (a) we have $f_{i}=f_{i}^{*}$ for $m>i \geq k$, in (b) we have $g_{i}=g_{i}^{*}$ for $i>k$, in (c) we have $A_{j}=A_{j}^{*}$, except for the case $k=m=n$ where we make $A_{n}=\left\{\alpha \in A_{n}^{*} \mid g_{n} \in \operatorname{Col}\left(\kappa_{n-1}^{++},<\alpha\right)\right\}$. (We needed this special case in order to satisfy clause (e) in the definition of the forcing conditions.) $F_{j}(\alpha)=F_{j}{ }^{*}(\alpha)$ for $j \geq n, \alpha \in A_{j}$, and in (d) $f_{i}=F_{i}{ }^{*}\left(\alpha_{i}\right)$ for $i \geq m, k$. (Note that if $p$ is a $k$-direct extension of $p^{*}$ then $p$ is determined by $p^{*}, p\lceil k$, and $\left\langle\alpha_{m}, \ldots, \alpha_{n-1}\right\rangle$; hence there are at most $\kappa_{\omega} k$-direct extensions of $\left.p.\right) p$ is a direct extension of $p^{*}$ if it is a 0 -direct extension and we also have $g_{0}=g_{0}^{*}$. Note that if $p$ is an extension of $p^{*}$ and given $k \leq n$, then we can get $p$ from $p^{*}$ by taking a $k$-direct extension of $p^{*}, s$, such that $p$ is a $k$-length-preserving extension of $s$. $s$ can be easily shown to be unique, and it will be called "the $k$ interpolant of $p^{*}$ and $p "$, denoted by $\operatorname{Int}\left(k, p^{*}, p\right)$.

The following lemma is typical for this class of forcing notion. It is known as the "Prikry Lemma". (See for instance [Gi], Claims 2, 3, 4 in $\S 3$ of this paper, or the main technical lemma in [Ma].) The proof is essentially along the lines of the quoted papers. At the insistence of the referee we include the proof.

Lemma 3. Let $D$ be an open subset of $\mathscr{P}$. Let $p \in \mathscr{P}$ be a condition of length $n$ and $k \leq n$; then there exists a $k$-length-preserving extension of $p, q$, such that

(a) if $q^{*} \leq q$ and $q^{*} \in D$, then $\operatorname{Int}\left(k, q, q^{*}\right) \in D$.

(b) If $q^{*} \leq q, q^{*} \in D$, and the length of $q^{*}$ is $m$, then any $q^{* *}$ satisfying $q^{* *} \leq q$, length $\left(q^{* *}\right)=m$, and $q^{* *}\left\lceil k=q^{*}\lceil k\right.$ is in $D$.

(c) If $D$ is the set of conditions deciding a given statement $\Phi$, we can assume that $q^{*} \Vdash \Phi$ iff $q^{* *} \Vdash \Phi\left(q^{*}\right.$ and $q^{* *}$ as above $)$.

$q$ satisfying the statements in Lemma 3 is said to be in $D$ modulo $k$-direct extensions. If $D$ is the dense set of conditions deciding the value of some term $\tau$, which is forced to denote an ordinal, then $q$ as above is said to determine $\tau$ up to $k$-direct extensions. If $D$ is the dense set of conditions deciding the truth value of some statement $\Phi$ in forcing language, then $q$ as above is said to decide $\Phi$ modulo $k$-direct extension. Note that in this case if $q^{*} \leq q q^{*}$ decides $\Phi$, then any $r$ which extends $q$ and satisfies $r\left\lceil k=q^{*}\lceil k\right.$ decides $\Phi$ in the same way. In particular the unique $r$ which is a $k$-length-preserving extension of $q$ and satisfies $r\left\lceil k=q^{*}\lceil k\right.$ decides $\Phi$. Namely for deciding $\Phi$ below $q$ we have only to change $q\lceil k$.

Proof of Lemma 3. We first prove a restricted version of Lemma 3, in which we get the conclusion for $q^{*}$ of fixed length.

Lemma 4. Let $p, n$, and $D$ be as in the statement of Lemma 3. Let $l$ be a natural number. Then there exists a $k$-length-preserving extension of $p, q$, such 
that for every extension of $q, q^{*}$ of length $n+l$, if $q^{*} \in D$ then $\operatorname{Int}\left(k, q, q^{*}\right) \in$ $D$.

Proof. We prove the lemma for every $p$ and $n$ by induction on $l$. There is a slight difference in the proof between the case $k<n$ and the case $k=n$. We shall deal with both cases simultaneously.

We start with the proof for $l=0$. The set $\{q\lceil k \mid q \leq p\}$ has cardinality $\alpha_{k}=\alpha_{k}(p)$ if $k<n$ and cardinality $\kappa_{n}$ if $k=n$. Define $\eta=\alpha_{k}$ if $k<n$ and $\eta=\kappa_{n}$ if $k=n$. Let $\left\langle r_{\delta} \mid \delta<\eta\right\rangle$ be an enumeration of this set. Put $r_{\delta}=\left\langle\alpha_{0}, \ldots, \alpha_{k-1}, g_{0}^{\delta}, \ldots, g_{k}^{\delta}, f_{0}^{\delta}, \ldots, f_{k-1}^{\delta}\right\rangle$. Without loss of generality we can assume that the enumeration is such that for $\delta$ inaccessible then $g_{k}^{\rho} \in$ $\operatorname{Col}\left(\kappa_{k-1}^{+2},<\delta\right)$ iff $\rho<\delta$. (Namely the enumeration restricted to $\delta$ enumerates exactly those $r_{\rho}$ mentioning only cardinals below $\delta$.)

By induction on $\delta<\eta$ we define a $\leq_{k}$ decreasing sequence of conditions $\left\langle q_{\delta} \mid \delta<\eta\right\rangle$ where $q_{0}=p$ and

$$
\begin{aligned}
q_{\delta}=\left\langle\alpha_{0}, \ldots, \alpha_{n-1},\left\langle A_{j}^{\delta} \mid j \geq n\right\rangle, g_{0}, \ldots, g_{k}, g_{k+1}^{\delta}, \ldots, g_{n}^{\delta},\right. \\
\left.f_{0}, \ldots, f_{k-1}, f_{k}^{\delta}, \ldots, f_{n-1}^{\delta},\left\langle F_{j}^{\delta} \mid j \geq n\right\rangle,\left\langle g_{j}^{\delta} \mid j>n\right\rangle\right\rangle .
\end{aligned}
$$

Note that this notation does not conflict with the notation we had above for the components of $r_{\delta}$. The notation uses the fact that $q_{\delta}$ is a $k$-length-preserving extension of $p$. For limit $\delta, q_{\delta}$ is a $\leq_{k}$ lower bound for $\left\langle q_{\rho} \mid \rho<\delta\right\rangle$. In order to define $q_{\delta+1}$ we "merge" $r_{\delta}$ and $q_{\delta}$ to form

$$
\begin{aligned}
s_{\delta}=\left\langle\alpha_{0}, \ldots, \alpha_{n-1},\left\langle A_{j}^{\delta} \mid j \geq n\right\rangle, g_{0}^{\delta}, \ldots, g_{n}^{\delta},\right. & f_{0}^{\delta}, \ldots, f_{n-1}^{\delta}, \\
& \left.\left\langle F_{j}^{\delta} \mid j \geq n\right\rangle,\left\langle g_{j}^{\delta} \mid j>n\right\rangle\right\rangle .
\end{aligned}
$$

If there is a $k$-length-preserving extension of $s_{\delta}$ of length $n, t_{\delta}$, such that $t_{\delta} \in D$, we define $q_{\delta+1}$ by putting, for $k \leq i, f_{i}^{\delta+1}=f_{i}\left(t_{\delta}\right)$. For $k<j<\omega$ we put $g_{j}^{\delta+1}=g_{j}\left(t_{\delta}\right)$, and for $n \leq j<\omega$ we put $F_{j}^{\delta+1}=F_{j}\left(t_{\delta}\right)$ and $A_{j}^{\delta+1}=$ $A_{j}\left(t_{\delta}\right)$. If no such $t_{\delta}$ exists, we define $q_{\delta+1}=q_{\delta}$.

The condition $q$, witnessing the truth of the present lemma, will be a $\leq_{k}$ lower bound of $\left\langle q_{\delta} \mid \delta<\eta\right\rangle$, if $k<n$. In the case $k=n, q$ is a "diagonal" $\leq_{k}$ lower bound to $\left\langle q_{\delta} \mid \delta<\eta\right\rangle$.

$$
\begin{aligned}
q=\left\langle\alpha_{0}, \ldots, \alpha_{n-1},\right. & \left\langle A_{j}^{*} \mid j \geq n\right\rangle, g_{0}, \ldots, g_{k}, g_{k+1}^{*}, \ldots, g_{n}^{*}, \\
& \left.f_{0}, \ldots, f_{k-1}, f_{k}^{*}, \ldots, f_{n-1}^{*},\left\langle F_{j}^{*} \mid j \geq n\right\rangle,\left\langle g_{j}^{*} \mid j>n\right\rangle\right\rangle
\end{aligned}
$$

where, for $k \leq i<n, f_{i}^{*}=\bigcup_{\delta<\eta} f_{i}^{\delta}$. For $k<j, g_{j}^{*}=\bigcup_{\delta<\eta} g_{j}^{\delta}$. For $j>n, A_{j}^{*}=\bigcap_{\delta<\eta} A_{j}^{\delta} . A_{n}^{*}$ is the diagonal intersection of the $A_{n}^{\delta}$, s. Namely, $A_{n}^{*}=\left\{\beta<\kappa_{n} \mid \beta \in \bigcap_{\delta<\beta} A_{n}^{\delta}\right\}$. For $\alpha \in A_{j}^{*}$ and $j>n, F_{j}^{*}(\alpha)=\bigcup_{\delta<\eta} F_{j}^{\delta} . F_{n}^{*}$ is defined by $F_{n}^{*}(\alpha)=\bigcup_{\delta<\alpha} F_{n}^{\delta}(\alpha)$.

This definition can be easily verified to give $q \in \mathscr{P}$. The proof that $\left[F_{j}^{*}\right]_{U_{j}} \in$ $G_{j}$ is as in Lemma 2, using the fact that $G_{j}$ is closed under unions of length 
$\leq \kappa_{j} . q$ is the required condition for the case $l=0$. If $q^{*} \leq q, q^{*}$ of length $n$, and $q^{*} \in D$, then $q^{*}\left\lceil k=r_{\delta}\right.$ for some $\delta<\eta . q^{*}$ is a $k$-length-preserving extension of $s_{\delta}$ which is in $D$. Hence $t_{\delta}$ exists. But $\operatorname{Int}\left(k, q, q^{*}\right)$ extends $t_{\delta}$; hence it is in $D$. The fact that $\operatorname{Int}\left(k, q, q^{*}\right) \leq t_{\delta}$ is straightforward in the case $k<n$. In the case $k=n$ we need that $A_{n}\left(\operatorname{Int}\left(n, q, q^{*}\right)\right) \subseteq A_{n}\left(t_{\delta}\right)=A_{n}^{\delta+1}$, but if $\mu$ is the sup of the inaccessibles below $\delta, g_{n}^{\delta}$ must mention some cardinal above $\mu$, by our assumption about the enumeration $\left\langle r_{\delta} \mid \delta<\eta\right\rangle$, so that every member of $A_{n}\left(\operatorname{Int}\left(n, q, q^{*}\right)\right)$ is above $\delta$. Since it is in $A_{n}^{*}$ and it is a limit ordinal, it is in $A_{n}\left(t_{\delta}\right)$. Similarly for $\alpha \in A_{n}\left(\operatorname{Int}\left(n, q, q^{*}\right)\right), F_{n}\left(t_{\delta}\right)(\alpha) \subseteq$ $F_{n}\left(\operatorname{Int}\left(n, q, q^{*}\right)\right)(\alpha)$.

We now deal with the induction step from $l$ to $l+1$. The set $\{(\alpha, f) \mid \alpha \in$ $\left.A_{n}, f \in \operatorname{Col}\left(\alpha^{+\omega+2},<\kappa_{n}\right)\right\}$ has cardinality $\kappa_{n}$. Enumerate this set in a sequence of length $\kappa_{n}:\left\langle\left(\alpha_{\delta}, f_{\delta}\right) \mid \delta<\kappa_{n}\right\rangle$. By induction on $\delta<\kappa_{n}$ we define an increasing sequence in $S_{n},\left\langle g_{j}^{\delta} \mid j \geq n+1\right\rangle$, where $g_{j}^{0}=g_{j}(p)$. For limit $\delta$ $\left\langle g_{j}^{\delta} \mid j \geq n+1\right\rangle$ is a lower bound of $\left\{\left\langle g_{j}^{\eta} \mid j \geq n+1\right\rangle \mid \eta<\delta\right\}$. For defining $g_{j}^{\delta+1}$ we consider the condition

$$
\begin{aligned}
p_{\delta}=\left\langle\alpha_{0}, \ldots, \alpha_{n-1}, \alpha_{\delta},\left\langle A_{j} \mid j \geq n+1\right\rangle,\right. \\
\left.\quad g_{0}, \ldots, g_{n}, g_{n+1}^{\delta}, f_{0}, \ldots, f_{n-1}, f_{\delta},\left\langle F_{j} \mid j \geq n+1\right\rangle,\left\langle g_{j}^{\delta} \mid j>n+1\right\rangle\right\rangle .
\end{aligned}
$$

$p_{\delta}$ has length $n+1$. By the induction assumption for $l$ applied to $p_{\delta}$, we can find $q_{\delta}$, which is a $k$-length-preserving extension of $p_{\delta}$ such that $q_{\delta}$ satisfies the conclusion of our lemma with respect to all $q^{*} \leq q_{\delta}$ provided the length of $q^{*}$ is $(n+1)+l=n+(l+1)$. We define, for $j \geq n+1, g_{j}^{\delta+1}=g_{j}\left(q_{\delta}\right)$. We also denote $f_{\delta}^{*}=f_{n}\left(q_{\delta}\right)$. $f_{\delta}^{*}$ clearly extends $f_{\delta}$. Given the inductive definition, we define, for $j \geq n+1, g_{j}^{*}=\bigcup_{\delta<\kappa_{n}} g_{j}^{\delta}$. Clearly

$$
g_{j}^{*} \in \operatorname{Col}\left(\kappa_{j-1}^{+2},<\kappa_{j}\right) .
$$

Fix $\alpha \in A_{n}$. Let $D_{\alpha}$ be the set of all conditions $h \in \operatorname{Col}\left(\alpha^{+\omega+2},<\kappa_{n}\right)$ such that there is a condition in $\mathscr{P}$ of the form

$$
\begin{array}{r}
\left\langle\alpha_{0}, \ldots, \alpha_{n-1}, \alpha,\left\langle A_{j}^{h} \mid j \geq n+1\right\rangle, g_{0}, \ldots, g_{k}, g_{k+1}^{h}, \ldots, g_{n}^{h}, g_{n+1}^{*}, *\right. \\
\left.f_{0}, \ldots, f_{k-1}, f_{k}^{h}, \ldots, f_{n-1}^{h}, h,\left\langle F_{j}^{h} \mid j \geq n+1\right\rangle,\left\langle g_{j}^{*} \mid j>n+1\right\rangle\right\rangle
\end{array}
$$

satisfying the conclusion of our lemma with respect to $q^{*}$ of length $n+(l+1)$. Fix $h \in \operatorname{Col}\left(\alpha^{+\omega+2},<\kappa_{n}\right)$. For some $\delta<\kappa_{n}$ we have $(\alpha, h)=\left(\alpha_{\delta}, f_{\delta}\right)$. By our construction $f_{\delta}^{*}$ extends $h$, and it is in $D_{\alpha}$. So we have shown that $D_{\alpha}$ is dense in $\operatorname{Col}\left(\alpha^{+\omega+2},<\kappa_{n}\right)$. It is clearly open. Consider the function $H(\alpha)=D_{\alpha}$ on $A_{n}$. Since $A_{n} \in U_{n}$, the equivalence class of $H$ modulo $U_{n}$, $[H]_{U_{n}}$, represents a member of $M_{n}$ which is (in the sense of $M_{n}$ ) a dense subset, $D^{*}$, of $\operatorname{Col}\left(\kappa^{+\omega+2},<j_{n}\left(\kappa_{n}\right)\right) . G_{n}$ is generic over $M_{n}$, so $D^{*} \cap G_{n} \neq \varnothing$. Let $[F]_{U_{n}} \in D^{*} \cap G_{n}$, and $[F]_{U_{n}}$ extends $\left[F_{n}\right]_{U_{n}}$. Without loss of generality we can assume that, for every $\alpha \in A_{n}, F(\alpha)$ extends $F_{n}(\alpha)$ and it is in $D_{\alpha}$. 
For $\alpha \in A_{n}$ let

$$
\begin{array}{r}
r_{\alpha}=\left\langle\alpha_{0}, \ldots, \alpha_{n-1}, \ldots,\left\langle A_{j}^{\alpha} \mid j \geq n+1\right\rangle, g_{0}, \ldots, g_{k}, g_{k+1}^{\alpha}, \ldots, g_{n}^{\alpha}, g_{n}^{*},\right. \\
\left.f_{0}, \ldots, f_{k-1}, f_{k}^{\alpha}, \ldots, f_{n-1}^{\alpha},\left\langle F_{j}^{\alpha} \mid j \geq n+1\right\rangle,\left\langle g_{j}^{*} \mid j>n+1\right\rangle\right\rangle
\end{array}
$$

be a witness to the fact that $F(\alpha) \in D_{\alpha}$. Namely for every $q^{*} \leq r_{\alpha}$ of length $n+l+1$, if $q^{*} \in D$ then $\operatorname{Int}\left(k, r_{\alpha}, q^{*}\right) \in D$. The sequence $X_{\alpha}=$ $\left\langle f_{k}^{\alpha}, \ldots, f_{n-1}^{\alpha}, g_{k+1}^{\alpha}, \ldots, g_{n}^{\alpha}\right\rangle$ can be coded as a bounded subset of $\alpha$. Hence by the normality of $U_{n}$ we get $C \subseteq A_{n}, C \in U_{n}$, such that this bounded set is fixed for $\alpha \in C$; namely for $\alpha \in C, X_{\alpha}=\left\langle f_{k}^{*}, \ldots, f_{n-1}^{*}, g_{k+1}^{*}, \ldots, g_{n}^{*}\right\rangle$ for some fixed sequence $\left\langle f_{k}^{*}, \ldots, f_{n-1}^{*}, g_{k+1}^{*}, \ldots, g_{n}^{*}\right\rangle$. For $\alpha \in C$ and $j>n$ we also have $F_{j}^{\alpha} \in G_{j} . G_{j}$ is $\kappa_{j}$ closed, so we can find $\left[F_{j}^{*}\right]_{U_{j}} \in G_{j}$ such that $\left[F_{j}^{*}\right]_{U_{j}} \leq\left[F_{j}^{\alpha}\right]_{U_{j}}$ for every $\alpha \in C$. We can also assume that $\left[F_{j}^{*}\right]_{U_{j}} \leq\left[F_{j}\right]_{U_{j}}$. We also define $F_{n}^{*}=F$. For $j>n$ we define

$$
A_{j}=\bigcap_{\alpha \in C} A_{j}^{\alpha} \cap \bigcap_{\alpha \in C}\left\{\beta \in A_{j} \mid F_{j}^{*}(\beta) \leq F_{j}^{\alpha}(\beta)\right\} \cap\left\{\beta \in A_{j} \mid F_{j}^{*}(\beta) \leq F_{j}(\beta)\right\} .
$$

Clearly $A_{j} \in U_{j}$. We also define $A_{n}^{*}=C$. We are ready to define the $k$ length-preserving extension of $p$ that will satisfy the present lemma for $l+1$. It is

$$
\begin{array}{r}
q=\left\langle\alpha_{0}, \ldots, \alpha_{n-1},\left\langle A_{j}^{*} \mid j \geq n\right\rangle, g_{0}, \ldots, g_{k}, g_{k+1}^{*}, \ldots, g_{n}^{*}, f_{0}, \ldots, f_{k-1},\right. \\
\left.f_{k}^{*}, \ldots, f_{n-1}^{*},\left\langle F_{j}^{*} \mid j \geq n\right\rangle,\left\langle g_{j}^{*} \mid j>n\right\rangle\right\rangle .
\end{array}
$$

$q$ satisfies the lemma for $l+1$ because, if $q^{*} \leq q, q^{*} \in D$, and the length of $q^{*}$ is $n+l+1$. Let $\alpha=\alpha_{n}\left(q^{*}\right)$. Of course $\alpha \in C . q^{*}$ can be easily seen to be an extension of $r_{\alpha}$, so, by the definition of $r_{\alpha}$, Int $\left(k, r_{\alpha}, q^{*}\right) \in D$, but $\operatorname{Int}\left(k, q, q^{*}\right)$ is an extension of $\operatorname{Int}\left(k, r_{\alpha}, q^{*}\right)$, so it is in $D$. This concludes the proof of Lemma 4.

Lemma 3 follows from Lemma 4 by applying Lemma $4 \omega$ times; namely we construct a $\leq_{k}$-decreasing sequence $\left\langle q_{k} \mid k<\omega\right\rangle$ such that $q_{0}=p$ and $q_{l+1}$ satisfies Lemma 4 for extensions of $q_{l+1}$ of length $n+l$. Let $r$ be a $\leq_{k}$ lower bound for the sequence. It satisfies clause (a) of Lemma 3 . We shall find a trivial extension of $r$ that will satisfy (b). Let $\left\langle B_{j} \mid j \geq n\right\rangle$ be the $A$-part of $r$. Let $T=\left\{q^{*}\left\lceil k \mid q^{*} \leq r\right\}\right.$. If $k<n$ then $|T|<\kappa_{n}$. If $k=n$ we put, for $\alpha \in A_{n}, T\left\lceil\alpha=\left\{q^{*}\left\lceil n \mid q^{*} \leq r, \alpha_{n}\left(q^{*}\right) \leq \alpha\right\}\right.\right.$. In this case $\mid T\lceil\alpha \mid=\alpha$. We define a partition of $\bigcup_{l \geq n} \prod_{j=n}^{l} B_{j}, \mathscr{F}$, where

$$
\begin{array}{r}
\mathscr{F}\left(\alpha_{n}, \ldots, \alpha_{l}\right)=\{s \in T \mid \text { the } k \text {-direct extension of } r \text { determined by } s \\
\text { and } \left.\alpha_{n}, \ldots, \alpha_{l} \text { is in } D\right\} .
\end{array}
$$

Note in the case $k=n, \mathscr{F}\left(\alpha_{n}, \ldots, \alpha_{l}\right) \subseteq T\left\lceil\alpha_{n}\right.$, so we can consider $\mathscr{F}\left(\alpha_{n}, \ldots, \alpha_{l}\right)$ to be essentially a subset of $\alpha_{n}$. Standard partition arguments, like Rowbottom's theorem, using the normality of the $U_{j}$ for $j \geq n$, give a sequence of sets $\left\langle C_{j} \mid j \geq n\right\rangle$ such that $C_{j} \in U_{j}$ (for $j \geq n$ ) and such that 
for $s \in T$ and $\left\langle\alpha_{n}, \ldots, \alpha_{l}\right\rangle \in \prod_{j=n}^{l} C_{j},\left\langle\beta_{n}, \ldots, \beta_{l}\right\rangle \in \prod_{j=n}^{l} C_{j}$ (in the case $k=n$ we also assume $s \in T\left\lceil\alpha_{n} \cap T\left\lceil\beta_{n}\right)\right.$

$$
s \in \mathscr{F}\left(\alpha_{n}, \ldots, \alpha_{l}\right) \text { iff } s \in \mathscr{F}\left(\beta_{n}, \ldots, \beta_{l}\right) \text {. }
$$

Let $q$ be the condition which is exactly like $r$ except that its $A$-part is $\left\langle C_{j}\right| j \geq$ $n\rangle$. $q$ clearly satisfies both (a) and (b) of Lemma 3. In order to get (c) (namely we are in the case that $D$ is the set of conditions deciding a sentence $\Phi$ ) we replace $D$ by $D^{*}$, the set of conditions forcing $\Phi$. A condition $q$ satisfying (a) and (b) for $D^{*}$ will satisfy (a), (b), and (c) for $D$.

Note that if $p$ and $k$ are as in Lemma $3(k<n)$ and we have a collection of $<\alpha_{k-1}(p)$ open subsets of $\mathscr{P}$, we can find $q$ satisfying Lemma 3 simultaneously for all the dense sets in our collection. Given a term $\tau$ which is forced to denote a subset of $\eta$ for some $\eta<\alpha_{k-1}(p)$, we can apply this observation to the collection of open subsets of $\mathscr{P},\left\{D_{\beta} \mid \beta<\eta\right\}$, where $D_{\beta}=\{r \in \mathscr{P} \mid r$ decides " $\beta \in \tau$ " $\}$. We get that an extension of $p$ forces that the realization of $\tau$ lies in $V[G\lceil k]$, where $G\lceil k=\{r\lceil k \mid r \in G$, the length of $r>k\} . G\lceil k$ is essentially a generic filter in

$$
\operatorname{Col}\left(\omega_{1},<\alpha_{0}\right) \times \prod_{j=1}^{k} \operatorname{Col}\left(\kappa_{j-1}^{+2},<\alpha_{j}\right) \times \prod_{j=0}^{k-1} \operatorname{Col}\left(\alpha_{j}^{+\omega+2},<\kappa_{j}\right)
$$

where $\left\langle\alpha_{0}, \alpha_{1}, \ldots\right\rangle$ is the union of all the $\alpha$-parts of conditions in the generic filter. As usual it follows from this remark that in the generic extension obtained by using $\mathscr{P}$ no cardinals below $\kappa_{\omega}$ are collapsed, unless we specifically provided for their collapse in our forcing notion. So the sequence of infinite cardinals below $\kappa_{\omega}$ is

$$
\begin{aligned}
\omega, \omega_{1}, \alpha_{0}, \alpha_{0}^{+}, \ldots, \alpha_{0}^{+\omega}, \alpha_{0}^{+\omega+1}, \alpha_{0}^{+\omega+2}, \kappa_{0}, \kappa_{0}^{+}, \kappa_{0}^{++}, \\
\alpha_{1}, \alpha_{1}^{+}, \ldots, \alpha_{1}^{+\omega}, \alpha_{1}^{+\omega+1}, \alpha_{1}^{+\omega+2}, \kappa_{2} .
\end{aligned}
$$

Clearly $\kappa_{\omega}$ in the extension is $\aleph_{\omega^{2}}$.

Since our forcing notion does not satisfy the $\lambda$ chain condition, where $\lambda=$ $\kappa_{\omega}^{+}$, we have to prove

Lemma 5. In $V^{P} \vDash \lambda$ is a cardinal.

Proof. We shall prove that $V^{\mathscr{P}}, \lambda$ is still regular. Assume otherwise, and let $p \in \mathscr{P}$ be a condition and $\tau$ a term such that

$$
p \Vdash \text { “ } \tau: \mu \rightarrow \lambda \text { cofinally where } \mu=\operatorname{cf}(\lambda) \text { " } \quad(\text { where } \mu<\lambda) .
$$

Since $\kappa_{\omega}$ is singular, $\mu<\kappa_{\omega}$. Since the sequence $\left\langle\alpha_{j} \mid j<\omega\right\rangle$ is cofinal in $\kappa_{\omega}$, $\mu<\alpha_{k}$ for some $k$. Without loss of generality we can assume that the length of $p>k$. Using Lemma 3 for the collection of $\left\langle D_{\beta} \mid \beta<\mu\right\rangle$ where $D_{\beta}$ is the collection of conditions deciding the value of $\tau(\beta)$, we get $q \leq p$ such that, for $\beta<\mu, q$ determines $\tau(\alpha)$ up to $k$-direct extensions. Since every possible value of $\tau(\beta)$ is determined by some $k$-direct extension of $q$, and since there are at most $\kappa_{\omega}$ such direct extensions, there are at most $\kappa_{\omega}$ possible values for 
$\tau(\beta)$. Hence once we are below $q$ there are at most $\kappa_{\omega}$ possible values for $\tau(\beta)$ for all $\beta<\mu$. Since this set of possible values is bounded in $\lambda$,

$$
q \Vdash \text { the range of } \tau \text { is bounded in } \lambda \text {, }
$$

which is a contradiction to $q \leq p$.

We are now left with the main task of the proof of Theorem 1, namely showing

$$
V^{\mathscr{P}} \vDash \Delta_{\kappa_{\omega}, \kappa_{\omega}^{+}}
$$

Let $\dot{\tau}, \dot{S}$ be terms, $\mu<\kappa_{\omega}$, and $p \in \mathscr{P}$ be a condition such that $p \Vdash \tau$ is an algebra on $\lambda$ with $\mu$ operations and $S$ is a stationary subset of $\lambda$. Since, for $\alpha<\lambda, \operatorname{cf}(\alpha)<\kappa_{\omega}$ and since forcing the conclusion of $\Delta_{\kappa_{\omega}, \kappa_{\omega}^{+}}$for a subset of $S$ instead of $S$ is enough, we can assume without loss of generality that

$$
p \Vdash \alpha \in S \rightarrow \operatorname{cf}(\alpha)<\kappa_{l}
$$

for some $l<\omega$. We can assume without loss of generality that also $\mu<\kappa_{l}$ and that the length of $p$ is $n$, where $l \leq n-1$.

The main idea of the rest of the proof is to argue in $V^{S_{n}}$, where we assume that the $S$ part of $p$ is in the $S_{n}$ generic filter $G\left(S_{n}\right)$. Recall that in $V^{S_{n}}$ $\kappa_{n}$ is still supercompact. In $V^{S_{n}}$ we shall get a "fake" version of $\dot{\tau}$ and of $\dot{S}$, and then use the supercompactness of $\kappa_{n}$ to get an elementary substructure of the "fake" $\dot{\tau} \dot{B}$, of a regular order type less than $\kappa_{n}$, such that $S \cap \mathscr{B}$ is stationary in $\sup (\mathscr{B})$. This seems almost a proof of $\Delta_{\kappa_{\omega}, \lambda}$, except that $\mathscr{B}$ is a substructure of the "fake" $\dot{\tau}$, not the real $\dot{\tau}$ as realized in $V^{\mathscr{P}}$, but the "fake" $\dot{\tau}$ and $\dot{\mathscr{B}}$ will be similar enough to their "real" counterparts that we will be able to extend $p$ to a condition in $\mathscr{P}$ forcing that $\mathscr{B}$ is an elementary substructure of the real $\dot{\tau}$ and that the "fake" $S \cap \mathscr{B}$ is a subset of the real $S$.

Before we start defining the "fake" $\dot{S}$ and $\dot{\tau}$, we have to normalize $\dot{S}$ somewhat. So for a little while let us argue inside $V^{\mathscr{P}}$. For every member $\beta \in S$ there exists some condition $q_{\beta}$ in the generic filter $G(\mathscr{P})$ such that $q_{\alpha} \Vdash \alpha \in \dot{S}$. Since there are less than $\lambda$ many possible lower parts of $q_{\beta}$, there is a stationary subset of $S, T$, such that for $\beta \in T$, the lower part of $q_{\beta}$ is fixed. Without loss of generality we can assume that the lower part of $p$ extends this fixed part; hence

$$
\begin{aligned}
p \Vdash T=\{\beta \mid \beta<\lambda, & \text { there is some } q \in G(\mathscr{P}), \\
& q \text { trivial extension of } p, q \Vdash \beta \in S\}
\end{aligned}
$$

is stationary in $\lambda$. The nice feature of $T$ is that if $q \leq p$ and $q \Vdash \alpha \in \dot{T}$ for some $\alpha<\lambda$, then, for some $n$-length-preserving extension of $p, q^{*}, q \leq q^{*} \leq$ $p, q^{*} \Vdash \alpha \in \dot{T}$.

We now move to $V^{S_{n}}$. In $V^{S_{n}}$ define $\mathscr{P}^{*}$ to be the set of $q \in \mathscr{P}$ such that length $(q) \geq n$ and its $S_{n}$ part belongs to $G\left(S_{n}\right)$. Partially order $\mathscr{P}^{*}$ as in $\mathscr{P}$. (Note that, by our assumption about $G\left(S_{n}\right), p \in \mathscr{P}^{*}$. We are slightly sloppy here, since if the length of $q$ is $k>n$ then its $S$-part formally does not belong 
to $S_{n}$ but to $S_{k}$, but we can naturally consider $S_{k}$ to be embedded into $S_{n}$ if $k>n$ in such a way that $G\left(S_{n}\right)$ naturally induces an $S_{k}$ generic filter.)

Lemma 6. Let $D \in V$ be a dense subset of $\mathscr{P}$. Then

(a) $V^{S_{n}} \vDash D \cap \mathscr{P}^{*}$ is a dense subset of $\mathscr{P}^{*}$.

(b) If $q \in \mathscr{P}^{*}, k \leq$ length $(q)$, then there is a $k$-length-preserving extension of $q, q^{*}, q^{*} \in \mathscr{P}^{*}$, such that $q^{*}$ satisfies the conclusions of Lemma 3.

This lemma implies that if we force with $\mathscr{P}^{*}$ over $V^{S_{n}}$ we introduce a $V$ generic filter for $\mathscr{P}$, so we can consider $V^{\mathscr{P}}$ to be a submodel of $V^{S_{n^{*}} \mathscr{P}^{*}}$.

Proof. (a) Let $r=\left\langle g_{i} \mid n-1<i<\omega\right\rangle$ be a member of $S_{n}$ such that $r \Vdash q \in \mathscr{P}^{*}$ but no extension of $q$ is in $D \cap \mathscr{P}^{*}$.

Let $q^{*}$ be an extension of $q$, such that the length of $q^{*}$ is $l \geq n$, such that its $S$-part extends $\left\langle g_{i} \mid l<i<\omega\right\rangle$. (It can be done since $r \Vdash q \in \mathscr{P}^{*}$.) Let $q^{* *}$ be an extension of $q^{*}$ in $D$ of length $m$. Let $\left\langle h_{i} \mid m<i<\omega\right\rangle$ be its $S$-part. Let $r^{*}=\left\langle g_{i}^{*} \mid n<i<\omega\right\rangle$ where $g_{i}^{*}=g_{i}$ for $i \leq m$ and $g_{i}^{*}=h_{i}$ for $i>m$. Clearly $r^{*} \Vdash q^{* *} \in \mathscr{P}^{*} \cap D$, which is a contradiction since $r^{*} \leq r$.

(b) Let $r$ be as above, $r \Vdash q \in \mathscr{P}^{*}$. Let $m=\operatorname{length}(q)$. Find an $m$-lengthpreserving extension of $q, q^{*}$, such that if the $S$-part of $q^{*}$ is $\left\langle h_{i} \mid m<i<\omega\right\rangle$ then $g_{i} \subseteq h_{i}$. Apply Lemma 3 to $q^{*}$ to get $q^{* *}$, a $k$-length-preserving extension of $q^{* *}$, which is in $D$ modulo $k$-direct extensions. Let $\left\langle h_{i}^{*} \mid m<i<\omega\right\rangle$ be the $S$-part of $q^{* *}$. Define $g_{i}^{*}=g_{i}$ for $n-1<i \leq m, g_{i}^{*}=h_{i}^{*}$ for $i>m$. $r^{*}=\left\langle g_{i}^{*} \mid n-1<i<\omega\right\rangle$ extends $r$ and forces $q^{* *}$ to be in $\mathscr{P}^{*}$.

We are now ready to define in $V^{S_{n}}$ our "fake" version of the stationary set $T, T^{*}$.

$T^{*}=\left\{\alpha \mid \alpha<\lambda\right.$, some trivial extension of $p$, which is in $\mathscr{P}^{*}$, forces $\left.\alpha \in \dot{T}\right\}$.

Let $q_{\alpha} \in \mathscr{P}^{*}\left(\alpha \in T^{*}\right)$ be a trivial extension of $p$ forcing $\alpha \in T . q_{\alpha}$ has of course the same lower part as $p$. Let $\left\langle A_{k}(\alpha) \mid k>n\right\rangle,\left\langle F_{k}(\alpha) \mid k>n\right\rangle,\left\langle g_{k}(\alpha)\right| k>$ $n\rangle$ be $q_{\alpha}$ 's $A$-part, $F$-part, and $S$-part respectively. For $\alpha \notin T^{*}$ make $q_{\alpha}=p$.

Lemma 7. $V^{S_{n}} \vDash T^{*}$ is a stationary subset of $\lambda$.

Proof. Assume otherwise, and let $\dot{C}$ be an $S_{n}$ term forced by $r \in S_{n}$ to be a closed unbounded subset of $\lambda$ disjoint from $T^{*}$. Define an equivalence relation $\approx$ on $S_{n}$ by $\left\langle g_{i} \mid n-1<i<\omega\right\rangle \approx\left\langle h_{i} \mid n-1<i<\omega\right\rangle$ if, for large enough $i, g_{i}=h_{i}$. For $r \in S_{n}$ let $[r]$ be its equivalence class. Let $V_{1}$ be $V\left[\left\{[r] \mid r \in G\left(S_{n}\right)\right\}\right]$. Clearly $V \subseteq V_{1} \subseteq V^{S_{n}}$. Clearly $V_{1}$ is obtained from $V$ by forcing, where the forcing notion is $S_{n} / \approx$, namely $\left\{[r] \mid r \in S_{n}\right\}$, where the partial order is defined by $\left[\left\langle g_{i} \mid n-1<i<\omega\right\rangle\right] \leq\left[\left\langle h_{i} \mid n<i<\omega\right\rangle\right]$ if, for large enough $i, h_{i} \subseteq g_{i}$.

Claim. $V^{S_{n}}$ is obtained from $V_{1}$ by a $\lambda$ c.c. forcing. Hence in $V_{1}$ there is a closed unbounded subset of $\lambda$ included in $C$.

Proof of the Claim. Let $G^{*}=\left\{[r] \mid r \in G\left(S_{n}\right)\right\}$. Clearly $V_{1}=V\left[G^{*}\right]$ and $G^{*}$ is $S_{n} / \approx$ generic over $V . V^{S_{n}}$ is obtained by forcing with $S_{n} / G^{*}=\left\{r \mid r \in S_{n}[r] \in\right.$ 
$\left.G^{*}\right\}$. So we really claim that $V_{1} \vDash S_{n} / G^{*}$ satisfies the $\lambda$ c.c. Let $\dot{D}$ be a term forced by [r] (over $S_{n} / \approx$ ) to be a maximal antichain in $S_{n} / G^{*}$. Let $Q_{m}$ be $\prod_{n-1 \leq i<m} \operatorname{Col}\left(\kappa_{i}^{+2},<\kappa_{i+1}\right)$. Note that the cardinality of $Q_{m}$ is $\kappa_{m}$; hence we can enumerate $\bigcup_{n-1<m<\omega} Q_{m}$ in a sequence of order type $\kappa_{\omega},\left\langle p_{\beta} \mid \beta<\kappa_{\omega}\right\rangle$, where, for $n \leq i, \kappa_{i}<\beta<\kappa_{i+1}, p_{\beta} \in Q_{i+1}$. Also, for $q \in S_{n}, p \in Q_{m}$ define $q * p$ to be the element of $S_{n}, q^{*}$, where $q^{*}(i)=p(i)$ for $n-1 \leq i<m$, and $q^{*}(i)=q(i)$ for $i \geq m$.

We now define by induction a decreasing sequence of elements of $S_{n},\left\langle r_{\beta}\right| \beta<$ $\left.\kappa_{\omega}\right\rangle$, such that if $\kappa_{i}<\beta, \gamma$ then, for $n<j \leq i, r_{\beta}(j)=r_{\gamma}(j)$. (Namely, above $\kappa_{i}$ we "freeze" the coordinates of $r_{\beta}$ below $i+1$.) $r_{0}$ is $r$. For limit $\beta$ define $r_{\beta}(i)=\bigcup_{\gamma<\beta} r_{\gamma}(i)$. It is a member of $S_{n}$ since if $\kappa_{j}<\beta \leq \kappa_{j+1}$ then if $j<i$ then $\operatorname{Col}\left(\kappa_{i}^{+2}<\kappa_{i+1}\right)$ is closed under unions of cardinality $\leq \kappa_{i}$ and, for $i \leq j, r_{\gamma}(i)$ is eventually constant below $\beta$. For defining the successor stage, namely $r_{\beta+1}$ where $r_{\beta}$ is given, consider the following statement:

$\Phi_{\beta}$ : There exists a member of $\dot{D}, q$, such that $q$ is compatible with $r_{\beta} * p_{\beta}$, where, if $p_{\beta} \in Q_{m}, q\left\lceil m=p_{\beta}\right.$.

(Note that if $p_{\beta} \in Q_{m}$, then $\beta<\kappa_{m}$.) Define $r_{\beta+1}$ to be a condition such that $\left[r_{\beta+1}\right]$ decides the truth of $\Phi_{\beta},\left[r_{\beta+1}\right] \leq\left[r_{\beta}\right]$, and moreover if $\left[r_{\beta+1}\right] \Vdash \Phi_{\beta}$ then, for some $q_{\beta}, q_{\beta}$ 「m= $p_{\beta}, q_{\beta}$ compatible with $r_{\beta} * p_{\beta}$.

$$
\left[r_{\beta+1}\right] \Vdash q_{\beta} \in S_{n} / G^{*} \wedge q_{\beta} \in D .
$$

Since $\left[r_{\beta+1}\right] \Vdash\left[q_{\beta}\right] \in G^{*}$, we must have $\left[r_{\beta+1}\right] \leq\left[q_{\beta}\right]$. Since we can replace $r_{\beta+1}$ by an equivalent condition and since, for $j>m, q_{\beta}(j), r_{\beta}(j)$ are compatible, we can assume that, for $j \leq m, r_{\beta+1}(j)=r_{\beta}(j)$ (hence since $\beta<\kappa_{m}$ our inductive assumption is satisfied) and, for $j>m, r_{\beta+1}(j) \supseteq q_{\beta}(j)$.

Let $r_{\infty}(i)=\bigcup_{\beta<\kappa_{\omega}} r_{\beta}(i)$. As before, it is easily checked that $r_{\infty} \in S_{n}$. Let $E=\left\{r_{\infty} * p \mid p \in \bigcup_{n-1<m<\omega} Q_{m}\right\}$. We claim that $\left[r_{\infty}\right] \Vdash_{S_{n} / \approx}$ 'Every element of $D$ can be extended to an element of $E$ '. Since $\dot{D}$ is supposed to be an antichain in $S_{n} / G^{*}$ and since $|E| \leq \kappa_{\omega}$ (note that $\left[r_{\infty}\right] \Vdash E \subseteq S_{n} / G^{*}$ ), this will prove that $|D| \leq \kappa_{\omega}$. Assume our last claim fails; hence we can find $s$ such that $[s] \leq\left[r_{\infty}\right]$ and $q$ such that $[s] \Vdash q \in \dot{D}$ but $q$ does not have an extension in $E$. Without loss of generality we can assume $s \leq r_{\infty}$. Also $[s] \Vdash[q] \in G^{*}$, and hence $[s] \leq[q]$. Hence there exists $m$ such that, for $m \leq i, s(i) \supseteq q(i)$. Consider $q\left\lceil m\right.$. It appears in our enumeration as some $p_{\beta}\left(\beta<\kappa_{m}\right)$. Consider the statement $\Phi_{\beta} . q$ clearly witnesses its truth; hence since $[s] \leq\left[r_{\beta+1}\right]$, we must have that $\left[r_{\beta+1}\right] \Vdash \Phi_{\beta}$. Hence we also get $\left[r_{\beta+1}\right] \Vdash q_{\beta} \in D$, where, for $j \leq m, q_{\beta}(j)=p_{\beta}(j)$ and, for $j>m, r_{\beta}(j) \supseteq q_{\beta+1}(j)$. Since, for $j>m$, $s(j) \supseteq r_{\infty}(j) \supseteq r_{\beta+1}(j)$ and $s(j) \supseteq q(j)$, we get that $s * p_{\beta} \leq q, s * p_{\beta} \leq q_{\beta}$ (as members of $S_{n}$ ), but $[s] \vDash s * p_{\beta} \in S_{n} / G^{*}$; hence

$[s] \vDash q$ and $q_{\beta}$ are in $\dot{D}$ and are compatible.

Since $\dot{D}$ is an antichain, we must have $q=q_{\beta}$, but then $r_{\infty} * p_{\beta} \leq r_{\beta+1} * p_{\beta} \leq$ 
$q_{\beta}=q$. Hence $q \in E$, which is a contradiction and proves the claim.

We resume the proof of Lemma 7. In view of the claim we can assume that the closed unbounded subset of $\lambda, C$, disjoint from $T^{*}$ lies in $V_{1}$. We force over $V^{S_{n}}$ with $\mathscr{P}^{*}$. By Lemma 6 we get a $\mathscr{P}$ generic filter over $V, G(\mathscr{P})$. In particular we have a realization of the set $T$, which in $V^{\mathscr{P}}$ is supposed to be stationary. By the definitions of $T$ and $T^{*}$ it is clear that $T \subseteq T^{*}$. We claim that $V_{1} \subset V^{\mathscr{P}}$. It is enough of course to show that $\left\{[r] \mid r \in G\left(S_{n}\right)\right\} \in V^{\mathscr{P}}$. Note that if $g \in \mathscr{P}$ then its $S$-part determines a unique element of $S_{n} / \approx$, denote it by $\bar{q}$, and since $G(\mathscr{P})$ is included in $\mathscr{P}^{*},\{\bar{q} \mid q \in G(\mathscr{P})\} \subseteq\left\{[r] \mid r \in G\left(S_{n}\right)\right\}$. A simple genericity argument also shows that if $[r] \in G\left(S_{n}\right)$ then, for some $q \in G(\mathscr{P}), \bar{q} \leq[r]$. (Assume otherwise; hence there is $q \in G(\mathscr{P})$,

$$
q \Vdash \text { for no } q^{*} \in G(\mathscr{P})\left[q^{*}\right] \leq[r] .
$$

But $q \in \mathscr{P}^{*}$, and hence $\bar{q}$ is in $\left\{[r] \mid r \in G\left(S_{n}\right)\right\}$. In particular $\bar{q}$ and $[r]$ are compatible. One can easily extend $q$ to $q^{*}$ such that $\bar{q}^{*} \leq[r]$, which is a contradiction.) Hence $\{[r] \mid$ for some $q \in G(\mathscr{P}), \bar{q} \leq[r]\}=\left\{[r] \mid r \in G\left(S_{n}\right)\right\}$. Therefore this set is in $V^{\mathscr{P}}$. In particular $C \in V^{\mathscr{P}}$, but $C \cap T^{*}=\varnothing$; hence $C \cap T=\varnothing$. We showed that $V^{\mathscr{P}} \vDash T$ is not stationary in $\lambda$, which is a contradiction.

So we already get in $V^{S_{n}}$ our version of the stationary set $T$, namely $T^{*}$. We are now going to construct our version of the algebra $\tau$. This algebra will be defined not in $V^{S_{n}}$ but in a forcing extension of it. Let $R_{n}^{*}$ be

$$
\underset{0 \leq i<n}{\times} \operatorname{Col}\left(\alpha_{i}^{+\omega+2}, \kappa_{i}\right) \times \underset{0 \leq i<n}{\times} \operatorname{Col}\left(\kappa_{i-1}^{++},<\alpha_{i}\right) \times \operatorname{Col}\left(\kappa_{n-1}^{++},<\kappa_{n}\right)
$$

(where $\left\langle\alpha_{0}, \ldots, \alpha_{n-1}\right\rangle$ is the $\alpha$-part of $p$ ). For technical simplicity we replace $R_{n}^{*}$ by an isomorphic partial order $R_{n}=\left\{\left\langle\alpha_{0}, \ldots, \alpha_{n-1}\right\rangle\right\} \times R_{n}^{*}$ (where $\left(\left\langle\alpha_{0}, \ldots, \alpha_{n}\right\rangle, r\right) \leq\left(\left\langle\alpha_{0}, \ldots, \alpha_{n}\right\rangle, r^{\prime}\right)$ if $r \leq r^{\prime}$ as members of $\left.R_{n}^{*}\right)$; the advantage of using $R_{n}$ is that if $q$ is of length $n$ then the lower part of $q$ is in $R_{n}$, and for the rest of this section, whenever we shall refer to an $R_{n}$ generic filter, we shall assume that it contains the lower part of $p$ as a member.

Recall that in $V^{S_{n}}$ we have defined $\mathscr{P}^{*} \subseteq \mathscr{P}$. In $V^{S_{n} \times R_{n}}$ we shall define $\mathscr{P}^{* *} \subseteq \mathscr{P}^{*} . q \in \mathscr{P}^{* *}$ if $q \in \mathscr{P}^{*}, q$ of length $n$, and the lower part of $q$ is in $G\left(R_{n}\right)$. Note that, by our assumptions on $G\left(S_{n}\right)$ and $G\left(R_{n}\right), p \in \mathscr{P}^{* *}$. Note that any two members of $\mathscr{P}^{* *}$ are compatible. (If $q_{1}, q_{2} \in \mathscr{P}^{* *}$ then their lower parts are compatible since both of them belong to $G\left(R_{n}\right)$. Their $S$-parts are compatible because they belong to $G\left(S_{n}\right)$. Any two $A$-parts and $F$-parts are compatible. It is obvious for the $A$-part. For the $F$-part use the fact that the equivalence class of $F_{k}\left(q_{1}\right)$ and $F_{k}\left(q_{2}\right)$ modulo $U_{j}$ belongs to the generic ultrafilter $G_{k}$. Hence

$$
B_{k}=\left\{\alpha \mid F_{k}\left(q_{1}\right)(\alpha) \text { is compatible with } F_{k}\left(q_{2}\right)(\alpha)\right\} \in U_{k}
$$

which lets us find a joint extension of the $A$-part and $F$-part of $q_{1}$ and $q_{2}$ respectively.) Actually using the countable closure of $S_{n} \times R_{n}$ one can show that if $q_{i}, i<\omega$, are in $\mathscr{P}^{* *}$, then they have a joint extension in $\mathscr{P}^{* *}$. 
Lemma 8. (a) Let $\Phi$ be a statement in the forcing language (over $V$ ) for $\mathscr{P}$; then there exists $r \in \mathscr{P}^{* *}, r \leq p$, such that $r$ decides $\Phi$.

(b) Let $D \subseteq \mathscr{P}$ be dense, $D \in V$; then there is $r \in \mathscr{P}^{* *}, r \leq p$, such that $r$ is in $D$ modulo direct extensions, and there exists $m$ such that any direct extension of $r$ of length $m$ is in $D$. (Note that, by the remarks above, any two $r$ 's in $\mathscr{P}^{* *}$ which decide $\Phi$ assign to $\Phi$ the same truth value.)

Proof. Let $\left(\left\langle h_{i} \mid i>n\right\rangle, t\right) \in S_{n} \times R_{n}$ force that the lemma fails, where we assume that $\left\langle h_{i} \mid i>n\right\rangle$ extends the $S$-part of $p$ and $t$ extends its lower part. Let $p^{*} \leq p$ be defined by specifying it to have $t$ as its lower part. Its $A$ part and $F$-part are like $p$, and its $S$-part is $\left.\left\langle h_{i} \mid i\right\rangle n\right\rangle$. Use Lemma 2 for $p^{*}$ and get $q^{*} \leq p^{*}$ having the same lower part as $p^{*}$, deciding $\Phi$ up to $n$ direct extensions. Let $r \leq q^{*}$ decide $\Phi$. By the remarks after Lemma 3 we can assume the length of $r$ is $n$. Let $\left\langle h_{i}^{*} \mid i>n\right\rangle, t^{*}$ be, respectively, the $S$-part and the lower part of $r ;\left(\left\langle h_{i}^{*} \mid i>n\right\rangle, t^{*}\right)$ clearly force that $r \in \mathscr{P}^{* *}$ and $r$ decides $\Phi$. Hence we get a contradiction since $\left.\left(\left\langle h_{i}^{*} \mid i\right\rangle n\right\rangle, t^{*}\right)$ extends $\left(\left\langle h_{i} \mid i>n\right\rangle, t\right)$. The proof of (b) is similar, using Lemma 3.

We are now ready to define the algebra $\tau^{*}$ in $V^{S_{n} \times R_{n}}$. Without loss of generality we assume that the type of $\tau$, namely the sequence of the cardinals specifying for each $n<\omega$ how many $n$-ary operations are in $\tau$, is in $V$. (We can achieve it, for instance, by assuming that $\tau$ has $\mu n$-ary operations for each $n<\omega$.) The algebra $\tau^{*}$ will be generated by the ordinals $<\lambda$, so each member of it will be a term (in the language appropriate for the type of $\tau)$ to a finite sequence of members of $\lambda$. $\tau^{*}$ will be completely determined if we specify for each two terms whether they denote the same member of the algebra (thus the elements of $\tau^{*}$ are essentially equivalence classes of terms). If $\rho$ and $\eta$ are terms and $\vec{\beta}, \vec{\gamma}$ sequences of ordinals $<\lambda$, then $\rho(\vec{\beta})=\eta(\vec{\gamma})$ if some $q \in \mathscr{P}^{* *}$ forces $\rho(\vec{\beta})=\eta(\vec{\gamma}) .(\rho(\vec{\beta})$ can be interpreted in $\tau$ to be some ordinal less than $\lambda$, similarly for $\eta(\vec{\gamma})$, so they are considered to be the same element in $\tau^{*}$ if some $q \in \mathscr{P}^{* *}$ forces these two ordinals to be the same.) Note that by Lemma 6 either there is $q \in \mathscr{P}^{* *}$ forcing $\rho(\vec{\beta})=\eta(\vec{\gamma})$ or there is $q \in \mathscr{P}^{* *}$ forcing $\rho(\vec{\beta}) \neq \eta(\vec{\gamma})$, and these two possibilities are exclusive.

We shall also define a well ordering of the elements of $\tau^{*}$. Namely $\rho(\vec{\beta})<$ $\eta(\vec{\gamma})$ if, for some $q \in \mathscr{P}^{* *}, q \Vdash \rho(\vec{\beta})<\eta(\vec{\gamma})$ as ordinals.

Lemma 9. $\tau^{*}$ is well ordered by the relation just defined.

Proof. Transitivity, antisymmetry, and reflexivity follow easily from the definition and from the fact that if $q_{1}, q_{2} \in \mathscr{P}^{* *}$ then $q_{1}, q_{2}$ are compatible in $\mathscr{P}^{* *}$. Given $\rho(\vec{\beta})$ and $\eta(\vec{\gamma})$, if there is $q \in \mathscr{P}^{* *}, q \Vdash \rho(\vec{\beta}) \neq \eta(\vec{\gamma})$, then there is $r \in \mathscr{P}^{* *}, r \Vdash \rho(\vec{\beta})<\eta(\vec{\gamma})$ or $r \Vdash \eta(\vec{\gamma})<\rho(\vec{\beta})$ (we cannot have $r$ forcing the negation of these two statements since such $r$ will be compatible with $q$ ). It is a well ordering because if $q_{i} \in \mathscr{P}^{* *}(i<\omega)$ forces $\rho_{i+1}\left(\vec{\beta}_{i+1}\right)<\rho_{i}\left(\vec{\beta}_{i}\right)$, then there is $q_{\infty} \in \mathscr{P}^{* *}$ extending all $q_{\infty}$ and then $q_{\infty} \Vdash \forall i\left(\rho_{i+1}\left(\vec{\beta}_{i+1}\right)<\rho_{i}\left(\vec{\beta}_{i}\right)\right)$, which is a contradiction.

We implicitly assumed that $\lambda$ is a subset of $\tau^{*}$, by having a trivial term $\beta$ 
which is identified with the ordinal $\beta$. Our next lemma shows that they are cofinal in $\tau^{*}$.

Lemma 10. For each term $\rho(\vec{\beta})$ there is $\gamma<\lambda$ such that, in $\tau^{*}, \rho(\vec{\beta})<\gamma$.

Proof. Note that $\mathscr{P}^{*}$ satisfies the $\lambda$ c.c. (Any two conditions in $\mathscr{P}^{*}$ agreeing on their lower part are compatible.) Hence the set $\left\{\delta \mid \delta<\lambda\right.$ some $q \in \mathscr{P}^{*}$ forces $\rho(\vec{\beta})=\delta\}$ is bounded in $\lambda$. Let $\gamma<\lambda$ be such a bound. If $q \in \mathscr{P}^{* *}$ then we cannot have $q \Vdash \rho(\vec{\beta})>\gamma$, because then by Lemma 6 there is $q^{*} \leq q$, $q^{*} \in \mathscr{P}^{*}, q \Vdash \rho(\vec{\beta})=\delta$. By definition of $\gamma$ we must have $\delta<\gamma$, hence a contradiction.

We shall now show that the order type of $\tau^{*}$ under the well ordering we have defined is $\lambda$. In view of Lemma 10 it will be enough to show that if $A_{\gamma}$ is the initial segment of $\tau^{*}$ determined by $\gamma<\lambda$, then $\left|A_{\gamma}\right| \leq \kappa_{\omega}$.

Lemma 11. $\left|A_{\gamma}\right| \leq \kappa_{\omega}$.

Proof. In $V \quad \gamma$ is an ordinal $<\lambda=\kappa_{\omega}^{+}$; hence there is a function in $V$, $f: \gamma \stackrel{1-1}{\rightarrow} \kappa_{\omega}$. Recall that $\kappa_{\omega}=\bigcup_{n<\omega} \kappa_{n}$; hence if $q \in \mathscr{P}^{* *}, q \Vdash \rho(\vec{\beta})<\gamma$, then $q \Vdash f(\rho(\vec{\beta})) \in \bigcup_{l<\omega} \kappa_{l}$. For each $l<\omega$ consider the statement $\Phi_{l}=$ " $f(\rho(\vec{\beta})) \in \kappa_{l}$ ". We have $q_{l} \in \mathscr{P}^{* *} ; q_{l}$ decides the truth value $\Phi_{l}$. We cannot have, for all $l<\omega, q_{l} \Vdash \neg \Phi_{l}$ because then we can find $q_{\infty} \leq q, q_{l}$ for $l<\omega$ but $q_{\infty} \Vdash f(\rho(\vec{\beta})) \in \bigcup_{l<\omega} \kappa_{l} \wedge \forall l\left(f(\rho(\vec{\beta})) \notin \kappa_{l}\right)$, which is clearly a contradiction. Hence, for every term in $A_{\gamma}, \rho(\vec{\beta})$, we can find some $l<\omega$ and $q \in \mathscr{P}^{* *}$ such that $q \Vdash f(\rho(\vec{\beta}))<\kappa_{l}$. So $A_{\gamma}=\bigcup_{l<\omega} A_{\gamma, l}$ where

$$
A_{\gamma, l}=\left\{\rho(\vec{\beta}) \mid \rho(\vec{\beta}) \in A_{\gamma}, \text { some } q \in \mathscr{P}^{* *} \text { forces } f(\rho(\vec{\beta}))<\kappa_{l}\right\} \text {. }
$$

It will be enough to show $\left|A_{\gamma, l}\right| \leq \kappa_{\omega}$. Assume otherwise. Let $\left\{\rho_{\delta}\left(\vec{\beta}_{\delta}\right) \mid \delta<\right.$ $\left.\kappa_{\omega}^{+}\right\}$be a list of $\kappa_{\omega}^{+}$different terms in $A_{\gamma, l}$. For each $\delta<\kappa_{\omega}$ let $D_{\delta}$ be the dense subset of $\mathscr{P}$ of those members of $\mathscr{P}$ which force $\rho_{\delta}\left(\vec{\beta}_{\delta}\right)=\zeta$ for some ordinal $\zeta$. By Lemma 6 we can find $q_{\delta} \in \mathscr{P}^{* *}, q_{\delta}$ as in $D_{\delta}$ up to direct extensions. (Note that then there is an $n \leq m_{\delta}<\omega$ such that any direct extension of $q_{\delta}$ of length $m_{\delta}$ is in $D_{\delta}$. .) Without loss of generality we can assume that

$$
q_{\delta} \Vdash \rho_{\delta}\left(\vec{\beta}_{\delta}\right)<\gamma \wedge f\left(\rho_{\gamma}\left(\vec{\beta}_{\delta}\right)\right)<\kappa_{l} .
$$

Also without loss of generality we can assume that $m_{\delta}$ is some constant $m$ (otherwise pass to a subset of $\left\{\rho_{\delta}\left(\vec{\beta}_{\delta}\right) \mid \delta<\kappa_{\omega}^{+}\right\}$of cardinality $\kappa_{\omega}^{+}$).

Given $\delta_{1}, \delta_{2}<\kappa_{\omega}^{+}$let $q_{\delta_{1}, \delta_{2}}$ be in $\mathscr{P}^{* *}$ :

$$
q_{\delta_{1}, \delta_{2}} \Vdash \rho_{\delta_{1}}\left(\vec{\beta}_{\delta}\right) \neq \rho_{\delta_{2}}\left(\vec{\beta}_{\delta_{2}}\right) .
$$

Without loss of generality we can assume that $q_{\delta_{1}, \delta_{2}} \leq q_{\delta_{1}}$ and $q_{\delta_{1} \delta_{2}} \leq q_{\delta_{2}}$. Let $q_{\delta_{1} \delta_{2}}^{*}$ be any direct extension of $q_{\delta_{1} \delta_{2}}$ of length $m \cdot q_{\delta_{1} \delta_{2}}^{*}$ clearly is in $\mathscr{P}^{*}$. 
Note that any two conditions in $\mathscr{P}^{*}$ having the same lower part are compatible. Also, for some $\xi_{1}^{\delta_{1}, \delta_{2}} \neq \xi_{2}^{\delta_{1}, \delta_{2}}, q_{\delta_{1} \delta_{2}}^{*} \Vdash \rho_{\delta_{1}}\left(\vec{\beta}_{\delta_{1}}\right)=\xi_{1}^{\delta_{1} \delta_{2}} \wedge \rho_{\delta_{2}}\left(\vec{\beta}_{\delta_{2}}\right)=\xi_{2}^{\delta_{1} \delta_{2}}$. Now define in $V^{S_{n+1} \times R_{n}}$ a partition on $[\lambda]^{2}:$ the two member subsets of $\lambda$. For $\delta_{1}<\delta_{2}$,

$$
g\left(\left\{\delta_{1}, \delta_{2}\right\}\right)=\left(\text { lower part of } q_{\delta_{1} \delta_{2}}^{*}\right) .
$$

Since $q_{\delta_{1} \delta_{2}}^{*}$ is of fixed length $m$, we have at most $\kappa_{m}$ many possible lower parts of $q_{\delta_{1} \delta_{2}}^{*}$. So the range of $g\left(\left\{\delta_{1}, \delta_{2}\right\}\right)$ is at most of cardinality $\kappa_{m}$, which is less than $\kappa_{\omega}$. (Note that since $R_{n}$ is of cardinality $<\kappa_{n}$ in $V^{S_{n}} \times R_{n}, \kappa_{\omega}$ is still a strong limit cardinal.) By the Erdös-Rado theorem (see [E-R]) we can find $A \subseteq \lambda,|A|>\kappa_{l}$, with the order type of $A$ a limit ordinal such that $g$ is constant on $[A]^{2}$. For $\delta_{1}, \delta_{2} \in A, q_{\delta_{1} \delta_{2}}^{*}$ are in $\mathscr{P}^{*}$, and they all have the same lower part; hence any two are compatible. So if $\delta_{1}<\delta_{2} \in A, \xi^{\delta_{1} \delta_{2}}$ does not depend on $\delta_{2}$, so denote it by $\xi^{\delta_{1}}$. Also, clearly $\xi^{\delta_{1}}<\gamma$ and $f\left(\xi^{\delta_{1}}\right)<\kappa_{l}$. It is also clear that if $\delta_{2}<\delta_{1}$ then $\xi_{2}^{\delta_{1} \delta_{1}}=\xi^{\delta_{1}}$; therefore, for $\delta_{1} \neq \delta_{2}$ in $A, \xi^{\delta_{1}} \neq \xi^{\delta_{2}}$, but then $f\left(\xi^{\delta_{1}}\right) \neq f\left(\xi^{\delta_{2}}\right)$ ( $f$ is $\left.1-1\right)$, and we get more than $\kappa_{l}$ different elements of $\kappa_{l}$, which is an obvious contradiction. So $\left|A_{\gamma, l}\right| \leq \kappa_{\omega}$.

We now step back to $V^{S_{n}}$, where $\tau^{*}$ will now be considered to be a $R_{n}$ term for an algebra on $\lambda$ forced to be well ordered by $<$ in order type $\lambda$. Abusing the notation we can also consider $\tau^{*}$ to be a term in $V^{S_{n}}$ for the forcing $R_{n}$. The main reason for moving down to $V^{S_{n}}$ is that, in $V^{S_{n}}, \kappa_{n}$ is still supercompact; hence we can find a normal ultrafilter $U^{*}$ on $P_{\kappa_{n}}(\lambda)$. By the choice of $U_{n}$ we can assume that $U^{*}$ projects to $U_{n}$. In $V^{S_{n}}$ consider the structure

$$
\mathscr{B}=\left\langle V_{\theta}, \lambda, T^{*}, \mathscr{P}^{*}, p, R_{n}, \tau^{*}\right\rangle
$$

for $\theta$ large enough. A standard use of the supercompactness of $\kappa_{n}$ (see for instance $[\mathrm{S}-\mathrm{R}-\mathrm{K}]$ or $[\mathrm{K}-\mathrm{M}]$ ) will prove

Lemma 12. The set

$$
\begin{array}{r}
E=\left\{P \in P_{k}(\lambda) \mid \text { for some elementary substructure of } \mathscr{B}, \mathscr{B}^{*}, P=\mathscr{B}^{*} \cap \lambda,\right. \\
\left.|P|=\left|\mathscr{B}^{* *}\right|, P \in \bigcap_{A \in U^{*} \cap \mathscr{B}^{*}} A\right\}
\end{array}
$$

is in $U^{*}$.

$T^{*}$ is a stationary subset of $\lambda$, all of its points having cofinality less than $\kappa_{n}$. Also, in $V^{S_{n}}, \lambda=\kappa_{n}^{+\omega+1}$. Another standard supercompactness argument shows that the set

$E_{1}=\left\{P \in P_{k}(\lambda) \mid P \cap \kappa_{n}\right.$ is an inaccessible cardinal, the order type of $P$ is $\left(P \cap \kappa_{n}\right)^{+\omega+1}, T^{*} \cap P$ is stationary in $\left.\sup (P)\right\}$

is in $U^{*}$. 
Lemma 13. Let $P \in E \cap E_{1} \cap A_{n}(p)$. Let $\mathscr{B}^{*}$ be an elementary substructure of $\mathscr{B}$ witnessing the fact that $P \in E$. Then there is a condition $q \in \mathscr{P}^{*}$ of length $n+1$, such that $\alpha_{n}(q)=P \cap \kappa_{n}$ and such that $q$ extends every trivial extension of $p$ which is in $\mathscr{P}^{*} \cap \mathscr{B}^{*}$.

Proof. Let $\alpha_{n}=P \cap \kappa_{n}$. We define $q$ as

$$
\begin{aligned}
\left\langle\alpha_{0}, \ldots, \alpha_{n},\left\langle A_{j}^{*} \mid n<j\right\rangle, g_{0}, \ldots, g_{n}, g_{n+1}^{*},\right. & f_{0}, \ldots, f_{n-1}, f_{n}^{*}, \\
& \left.\left\langle F_{j}^{*} \mid n<j\right\rangle,\left\langle g_{j}^{*} \mid n+1<j\right\rangle\right\rangle .
\end{aligned}
$$

(We have used the superscript * for every component of $q$ which is different than the corresponding component of $p$.)

$A_{j}^{*}$ is the intersection $\bigcap_{A \in U_{j} \cap \mathscr{B}^{*}} A$. It is in $U_{j}$ (for $j>n$ ) since the cardinality of $\mathscr{B}^{*}$ is less than $\kappa_{n}<\kappa_{j}$. (Note that $\mathscr{B}^{*}$ is in $V^{S_{n}}$, but since $S_{n}$ is $\kappa_{n}$ closed, $\mathscr{B}^{*} \cap V$ is in $V$; hence we intersect a family of sets in $V$.) Similarly $g_{j}^{*}$ for $j>n$ is defined to be the union of the $j$ th components of $G\left(S_{n}\right) \cap \mathscr{B}^{*}$. Again the fact that $S_{n}$ is $\kappa_{n}$ closed guarantees that this union is in $V$, and it is in $\operatorname{Col}\left(\kappa_{j-1}^{++},<\kappa_{j}\right)$.

$f_{n}^{*}$ is defined to be $\bigcup\left\{F\left(\alpha_{n}\right) \mid F \in \mathscr{B}^{*},[F]_{U_{n}} \in G_{n}\right\}$. We have to show that $f_{n}^{*}$ is in $\operatorname{Col}\left(\alpha_{n}^{+\omega+2},<\kappa_{n}\right)$. We assumed that for every $A \in U_{n}^{*} \cap \mathscr{B}^{*}$ we have $P \in A$; hence, since $U_{n}$ is the projection of $U_{n}^{*}$, we get that, for $A \in U_{n} \cap \mathscr{B}^{*}$, $P \cap \kappa_{n}=\alpha_{n} \in A$. If $F$ and $F^{*}$ are in $\mathscr{B}^{*}$ and $[F]_{U_{n}},\left[F^{*}\right]_{U_{n}} \in G_{n}$, we get $X=\left\{\beta<\kappa_{n} \mid F(\beta), F^{*}(\beta) \in \operatorname{Col}\left(\beta^{+\omega+2},<\kappa_{n}\right), F(\beta), F^{*}(\beta)\right.$ are compatible $\}$ $\in U_{n}$. If $F, F^{*} \in \mathscr{B}^{*}$, we have $X=U_{n} \cap \mathscr{B}^{*}$, so $\alpha_{n} \in X$. Therefore $f_{n}$ is the union of $\left|\mathscr{B}^{*}\right|$ mutually compatible conditions in $\operatorname{Col}\left(\alpha_{r}^{+\omega+2},<\kappa_{n}\right)$, and as before this union is in $V \cdot\left|\mathscr{B}^{*}\right|=|P|=\alpha_{n}^{+\omega+1}$; hence this union is in $\operatorname{Col}\left(\alpha_{n}^{+\omega+2},<\kappa_{n}\right)$.

By the way, this is the point that blocked us from collapsing any cardinals between $\alpha_{n}$ and $\alpha_{n}^{+\omega+1}$, and it is the reason that the proof of Theorem 1 does not work for cardinals less than $\aleph_{\omega^{2}+1}$.

For $j>n$ and $\beta \in A_{j}^{*}$ we define $F_{j}^{*}(\beta)=\bigcup\left\{F(\beta) \mid F \in \mathscr{B}^{*},[F]_{U_{j}} \in G_{j}\right\}$. An argument exactly like the one for $f_{n}$ shows that $F_{j}^{*}(\beta) \in \operatorname{Col}\left(\beta^{\omega+2},<\kappa_{j}\right)$ and that $\left[F_{j}^{*}\right]_{U_{j}} \in G_{j}$. We have proved that $q$ is a condition. The fact that $q$ extends every trivial extension of $p$ in $\mathscr{P}^{*} \cap \mathscr{B}^{*}$ follows immediately from the definition of $q$.

We are now ready to verify $\Delta_{\aleph_{\omega^{2}}, \aleph_{\omega^{2}+1}}$ is in $V^{\mathscr{P}}$. Let $P \in E \cap E_{1}$, and let $\mathscr{B}^{*}$ witness that $P \in E$. Let $\alpha={ }^{\infty} \cap \kappa_{n}=\mathscr{B}^{*} \cap \kappa_{n}$. Let $q$ be as in Lemma 13 . We claim that $q$ forces that the subalgebra of $\tau$ generated by $P$ has the same order type as $P$ and $P$ is cofinal in it. Note that $q$ forces that the order type of $P$ is a regular cardinal in $V^{\mathscr{P}}$, since $\alpha_{n}(q)=P \cap \kappa_{n}$, the order type of $P$ is $\left(P \cap \kappa_{n}\right)^{+\omega+1}$, and no cardinals are collapsed between $\alpha_{n}$ and $\alpha_{n}^{+\omega+2}$. Also note that, for $\beta \in T^{*} \cap P$, some trivial extension of $p$ in $\mathscr{P}^{*}$ forces $\beta \in T$, 
but by $\mathscr{B}^{*}$ being an elementary substructure of $\mathscr{B}$, such an extension is in $\mathscr{B}^{*}$, so $q$ extends it; hence $q$ forces that $\beta \in T$. Since we have $P \in E_{1}$, $P \cap T^{*}$ is stationary in $\sup (P)$, we get that $q$ forces that $T \cap P$ is stationary in $\sup (P)$. So if we verify the claim, we get that $q$ forces that the subalgebra generated by $P$ is a witness to $\Delta_{\aleph_{\omega^{2}}, \aleph_{\omega^{2}+1}}$. Note that we use the fact that, while the argument takes place in $V^{S_{n}}, P$ and $q$ are in $V$ so the witnesses are in $V$.

Claim. $q$ forces that the subalgebra of $\tau$ generated by $P$ has order type $|P|$ and $P$ is cofinal in it.

Proof of the claim. Let $\mathscr{A}$ be the subalgebra of $\tau$ generated by $P . \tau^{*}$ is an algebra in $\left(V^{S_{n}}\right)^{R_{n}}$ of order type $\lambda$, so let $h$ be an $R_{n}$ term in $V^{S_{n}}$ forced to be an order-preserving map from $\tau^{*}$ onto $\lambda . R_{n}$ satisfies $\kappa_{n}$ c.c. Hence for every term $\rho(\vec{\eta})$ there is a set of cardinality less than $\kappa_{n}, X=X_{\rho(\vec{\eta})}$, such that every condition in $R_{n}$ forces that $h(\rho(\vec{\eta})) \in X$. If $\vec{\eta} \in P$ then by $P \cap \lambda=\mathscr{B}^{*}$ we get that $X \subseteq P$. We have shown that the subalgebra of $\tau^{*}$ generated by $P$ has order type $|P|$. A similar argument shows that $P$ is cofinal in it. Also, using again the $\kappa_{n}$ c.c. of $R_{n}$, we get that the subalgebra of $\tau^{*}$ generated by $P$ (we denote it by $\mathscr{A}^{*}$ ) is in $\left(V^{S_{n}}\right)^{R_{n}^{\alpha}}$, where $R_{n}^{\alpha}$ is $\times_{0 \leq i<n} \operatorname{Col}\left(\alpha_{i}^{+\omega+2}, \kappa_{i}\right) \times \times_{0 \leq i<n}\left(\kappa_{i-1}^{+2},<\alpha_{i}\right) \times \operatorname{Col}\left(\kappa_{n-1},<\alpha\right)$. Since $S_{n}$ is $\kappa_{n}$ closed, $\mathscr{A}^{*}$ is in $V^{R_{n}^{\alpha}}$. Forcing with $\mathscr{P}$ (provided $q \in G(\mathscr{P})$ ) introduces a generic filter for $R_{n}^{\alpha}$. (We denote this generic filter by $G\left(R_{n}^{\alpha}\right)$.) Hence we can assume that $\mathscr{A}^{*}$ is in $V^{\mathscr{P}}$. Our claim will be finished if we show that $q$ forces that $\mathscr{A}$ is isomorphic (including the order) to $\mathscr{A}^{*}$ by an isomorphism which is the identity on $P$. The isomorphism is quite clear. A member of $\mathscr{A}$ has the form $\rho(\vec{\eta})$ where $\rho$ is a term in the signature of $\tau$ and $\vec{\eta}$ is a sequence of members of $P$. We map $\rho(\vec{\eta})$ to the equivalence class of this term in $\tau^{*}$. In order to show that this is a well-defined map which is an isomorphism we have to show that if $\rho_{1}, \rho_{2}$ are two terms in the signature of $\tau$ applied to some members of $P$ (we do not mention these members of $P$ explicitly) and if $\rho_{1}=\rho_{2} \quad\left(\rho_{1}<\rho_{2}\right)$ in the sense of $\mathscr{A}^{*}$ then $\rho_{1}=\rho_{2} \quad\left(\rho_{1}<\rho_{2}\right)$ in the sense of $\mathscr{A}$. But $\rho_{1}=\rho_{2}$ (in the sense of $\mathscr{A}^{*}$ ) means that for some $r$ which is a trivial extension of $p$ and some $t \in G\left(R_{n}^{\alpha}\right)$, if $r^{*}$ is the condition which is like $r$ except that its lower part is $t$, then $r^{*}$ forces $\rho_{1}=\rho_{2}$. By $\mathscr{B}^{*}$ being an elementary substructure of $\mathscr{B}$ we know that we can assume that $r \in \mathscr{B}^{*}$; hence $q$ extends $r$. Hence $r \in G(\mathscr{P})$. By definition of $G\left(R_{n}^{\alpha}\right)$ in $V^{\mathscr{P}}$ we know that $r^{*} \in G(\mathscr{P})$; hence $\rho_{1}=\rho_{2}$ in the sense of $\tau$. The argument in the case $\rho_{1}<\rho_{2}$ (in the sense of $\mathscr{A}^{*}$ ) is similar.

This completes the proof of Theorem 1.

For future references in the next section we need to consider the problem of modifying our forcing construction, so as to get $\Delta_{\kappa_{\omega}, \rho}$ for all regular $\mu \geq$ $\rho>\kappa_{\omega}^{+}$for some $\mu$. Essentially the only facts specific to $\kappa_{\omega}^{+}$in the proof of Theorem 1 were that $U_{n}(n<\omega)$ was forced by $S_{n}$ to be the projection 
of a normal ultrafilter on $P_{\kappa_{n}}\left(\kappa_{\omega}^{+}\right)$, in the forcing extension $V^{S_{n}}, F_{n}$, and that $\left\{P \mid P \in P_{\kappa_{n}}\left(\kappa_{\omega}^{+}\right)\right.$, the order type of $\left.P=|P \cap \kappa|^{+\omega+1}\right\} \in F_{n}$. (Note that $\{P \mid$ order type $\left.(P) \leq|P \cap \kappa|^{+\omega+1}\right\} \in F$ is enough for the proof of Theorem 1.) So suppose that we are given a cardinal $\mu>\kappa_{\omega}$ and a sequence of ultrafilters $\left\langle U_{n} \mid n<\omega\right\rangle$ and a sequence of functions $\left\langle h_{n} \mid n<\omega\right\rangle, h_{n}: \kappa_{n} \rightarrow \kappa_{n}$, such that every condition in $S_{n}$ forces that $U_{n}$ is the projection of a normal ultrafilter $F_{n}$, on $P_{\kappa_{n}}(\mu)$, such that

$$
\left\{P \mid P \in P_{\kappa_{n}}(\mu), \text { the order type of } P \leq h_{n}\left(P \cap \kappa_{n}\right)\right\} \in F_{n} .
$$

(Note that $U_{n}$ is then forced to be the projection of a normal ultrafilter on $P_{\kappa_{n}}(\rho)$ for all $\rho \leq \mu$.) We can then define an analogue of our forcing notion $\mathscr{P}$, where the only difference in the definition is that we require $f_{i} \in$ $\operatorname{Col}\left(h_{i}\left(\alpha_{i}\right)^{+},<\kappa_{i}\right)$ (in our previous argument $h_{i}\left(\alpha_{i}\right)=\alpha_{i}^{+\omega+1}$ ), and similarly $F_{i}(\delta) \in \operatorname{Col}\left(h_{i}(\delta)^{+},<\kappa_{i}\right)$. The proofs of Lemmas 2-4 are as before; Lemma 5 should now read "All cardinals $\rho \leq \mu, \rho>\kappa_{\omega}$, are preserved". The proof for $\rho>\kappa_{\omega}^{+}$simply follows from the fact that, since we assume G.C.H. above $\kappa_{\omega}$, the cardinality of $\mathscr{P}$ is $\kappa_{\omega}^{+}$. (We cannot claim now that $\kappa_{\omega}$ is $\aleph_{\omega^{2}}$. The index of $\kappa_{\omega}$ in the $\aleph$ sequence depends of course on the functions $\left\langle h_{i} \mid i<\omega\right\rangle$, because they determine how many cardinals we leave uncollapsed.)

We claim that in $V^{\mathscr{P}}$ we have $\Delta_{\kappa_{\omega}, \rho}$ for all regular $\rho \leq \mu$. So again we are given terms $\dot{\tau}, \dot{S}$ for an algebra $\dot{\tau}$ on $\rho$ and a stationary subset of $\rho, \dot{S}$. We are also given a condition $p$ of length $n$. We assume that if $\rho=\eta^{+}$, where $\eta$ is singular of cofinality less than $\kappa_{\omega}$, then $\operatorname{cf}(\eta)<\kappa_{n-1}$. The definitions of $T, T^{*}$ in $V^{S_{n}}$ and the proof that $T^{*}$ is stationary in $V^{S_{n}}$ go as before. (They are actually simpler if $\rho>\kappa_{\omega}^{+}$, because $|\mathscr{P}|=\kappa_{\omega}^{+}$.) Also the definition of the algebra $\tau^{*}$ in $V^{S_{n} \times R_{n}}$ is as in the proof of Theorem 1. The only proof that requires modification is the proof of Lemma 11 , namely showing that

Lemma 14. In our modified situation $\left|A_{\gamma}\right|<\rho \quad($ for $\gamma<\rho)$.

Proof. We distinguish two cases.

Case I: $\rho$ is inaccessible or it is the successor of a cardinal whose cofinality is $>\kappa_{\omega}$. Note that by G.C.H. holding above $\kappa_{\omega},\left|\gamma^{\kappa_{\omega}}\right|<\rho$. As in the proof of Lemma 9 let $\left\{\rho_{\delta}\left(\vec{\beta}_{\delta}\right) \mid \delta<\rho\right\}$ be a sequence of terms, such that, for $\delta_{1}, \delta_{2}<\rho$,

$$
\exists q_{\delta_{1} \delta_{2}} \in \mathscr{P}^{* *} \quad q_{\delta_{1} \delta_{2}} \Vdash \rho_{\delta_{1}}\left(\vec{\beta}_{\delta_{1}}\right)<\gamma \wedge \rho_{\delta_{2}}\left(\vec{\beta}_{\delta_{2}}\right)<\gamma \wedge \rho_{\delta_{1}}\left(\vec{\beta}_{\delta_{1}}\right) \neq \rho_{\delta_{2}}\left(\vec{\beta}_{\delta_{2}}\right) .
$$

For $\delta<\rho$ let $q_{\delta} \in \mathscr{P}^{* *}$ be in $D_{\delta}$ up to direct extensions (where $D_{\delta}$ are the dense subsets of those members of $\mathscr{P}$ which force $\rho_{\delta}\left(\beta_{\delta}\right)=\zeta$ for some $\zeta<\rho$ ), and without loss of generality assume $q_{\delta} \Vdash \rho_{\delta}\left(\vec{\beta}_{\delta}\right)<\gamma$. Every direct extension of $q_{\delta}$ is determined by some $\alpha_{n+1}, \ldots, \alpha_{m}<\kappa_{\omega}$. So define $H_{\delta}: \kappa_{\omega}^{<\omega} \rightarrow \gamma$ by $H_{\delta}\left(\alpha_{n+1}, \ldots, \alpha_{m}\right)=$ the unique $\zeta+1$ such that the direct extension of $q_{\delta}$ determined by $\alpha_{n+1}, \ldots, \alpha_{m}$ forces $\rho_{\delta}\left(\vec{\beta}_{\delta}\right)=\zeta$ if such a direct extension exists, and 0 otherwise. $H_{\delta}$ is essentially a function from $\kappa_{\omega}$ into $\gamma$; hence 
by our assumption we have less than $\rho$ such functions. Therefore we can find $\delta_{1}, \delta_{2}<\rho, \delta_{1} \neq \delta_{2}$, such that $H_{\delta_{1}}=H_{\delta_{2}}$. It immediately follows that every condition extending $q_{\delta_{1}}$ and $q_{\delta_{2}}$ forces $\rho_{\delta_{1}}\left(\vec{\beta}_{\delta_{1}}\right)=\rho_{\delta_{2}}\left(\vec{\beta}_{\delta_{2}}\right)$, which contradicts the fact that $q_{\delta_{1}}, q_{\delta_{2}}$, and $q_{\delta_{1}, \delta_{2}}$ are compatible.

Case II: $\rho=\eta^{+}$where $\operatorname{cf}(\eta)<\kappa_{\omega}$. Hence $\operatorname{cf}(\eta)<\kappa_{l}$ for some $l$, and we assume that we have $l<n$. As in the proof of Lemma 11 we pick a oneto-one function $f, f: \gamma \rightarrow \eta$. Also let $\eta=\sup \left\langle\eta_{\zeta} \mid \zeta<\operatorname{cf}(\eta)\right\rangle$. Again let $\left\langle\rho_{\delta}\left(\vec{\beta}_{\delta}\right) \mid \delta<\rho\right\rangle$ be a counterexample to the fact we are trying to prove.

Claim. For each $\delta<\rho, \exists q_{\delta} \in \mathscr{P}^{* *}$ and $\zeta(\delta)<\operatorname{cf}(\eta)$ such that $q_{\delta} \Vdash f\left(\rho_{\delta}\left(\vec{\beta}_{\delta}\right)\right)$ $\in \eta_{\zeta(\delta)}$.

Proof. Assume otherwise; let $\left.\left\langle\left\langle h_{i} \mid i\right\rangle n\right\rangle, t\right\rangle \in S_{n} \times R_{n}$ force that the claim fails, where we assume that $\left\langle h_{i} \mid i>n\right\rangle$ extends the $S$-part of $p$ and $t$ extends the lower part of $p$. Let $p^{*}$ have $t$ as a lower part, $\left\langle h_{i} \mid i>n\right\rangle$ as its $S$-part, and be otherwise like $p$. Let $\Phi_{\zeta}(\zeta<\operatorname{cf}(\eta))$ be the statement " $f\left(\rho_{\delta}\left(\vec{\beta}_{\delta}\right)\right) \in \eta_{\zeta}$ ", By the remarks following Lemma 3 (using $\operatorname{cf}(\eta)<\kappa_{n-1}$ ) we can get an $n$ length-preserving extension of $p^{*}, q$, such that $q$ decides $\Phi_{\zeta}$ modulo $n$-direct extension for all $\zeta<\operatorname{cf}(\eta)$ and satisfies (c) of Lemma 3 with respect to each $\Phi_{\zeta}$. Since we must have " $f\left(\rho_{\delta}\left(\vec{\beta}_{\delta}\right)\right) \in \eta_{\zeta}$ " for some $\zeta<\operatorname{cf}(\eta)$, let $q^{*}$ be an extension of $q$ forcing " $f\left(\rho_{\delta}\left(\vec{\beta}_{\delta}\right)\right) \in \eta_{\zeta}$ ” for some $\zeta<\operatorname{cf}(\eta)$. By the properties of $q, q^{*}$ can be assumed to be an $n$-direct extension of $q$ of length $n$. Let $\left\langle h_{i}^{*} \mid i>n\right\rangle$ be the $S$-part of $q^{*}$ and $t^{*}$ its lower part. Clearly $\left\langle\left\langle h_{i}^{*} \mid i>n\right\rangle, t^{*}\right\rangle$ extends $\left\langle\left\langle h_{i} \mid i>n\right\rangle, t\right\rangle$ and forces $q^{*} \in \mathscr{P}^{* *}$, but $q^{*}$ can serve as $q_{\delta}$ in the claim and $\zeta$ as $\zeta_{(\delta)}$, a contradiction.

Without loss of generality we can assume that $\zeta(\delta)$ is the constant $\zeta$ for $\delta<\rho$. Now the proof is like the proof of Lemma 11; namely for $\delta<\rho$ we pick $q_{\delta} \in \mathscr{P}^{* *}$,

$$
q_{\delta} \Vdash \rho_{\delta}\left(\vec{\beta}_{\delta}\right)<\gamma \wedge f\left(\rho_{\delta}\left(\vec{\beta}_{\delta}\right)\right)<\eta_{\zeta},
$$

and $q_{\delta}$ is in $D_{\delta}$ up to direct extensions (where $D_{\delta}$ is the dense set of members of $P$ forcing a specific value to $\rho_{\delta}\left(\vec{\beta}_{\delta}\right)$ ), and such that getting into $D_{\delta}$ we have simply to take a direct extension of $q_{\delta}$ of length $m_{\delta}$ (which without loss of generality we can assume is a constant $m$.) Again we pick for $\delta_{1}, \delta_{2}<\rho$, $q_{\delta_{1} \delta_{2}} \in \mathscr{P}^{* *}, q_{\delta_{1} \delta_{2}}$ extending $q_{\delta_{1}}, q_{\delta_{2}}$ and forcing $\rho_{\delta_{1}}\left(\vec{\beta}_{\delta_{1}}\right) \neq \rho_{\delta_{2}}\left(\vec{\beta}_{\delta_{2}}\right)$.

Defining the partition $g\left(\delta_{1}, \delta_{2}\right)=$ lower part of $q_{\delta_{1} \delta_{2}}$ and using the ErdösRado theorem again (remember that $\eta$ is strong limit) to get a homogeneous set for $g$ of cardinality $>\eta_{\zeta}$ yield a contradiction as in the proof of Lemma 11.

From now on, the proof of $\Delta_{\kappa_{\omega}, \rho}$ is exactly as in the proof of Theorem 1. Thus we proved

Theorem 15. Assume $\left\langle\kappa_{n} \mid n<\omega\right\rangle$ is a sequence of cardinals. $\kappa_{\omega}=\sup \left\langle\kappa_{n}\right| n<$ $\omega\rangle, \mu>\kappa_{\omega}$. Assume that G.C.H. holds above $\kappa_{\omega}$ and $2^{\kappa_{n}}=\kappa_{n}^{+}$. Assume also 
that $\kappa_{n}$ is $\mu$ supercompact if we force with $S_{n}$. Let $\left\langle f_{n} \mid n<\omega\right\rangle$ be a sequence of functions $f_{n}: \kappa_{n} \rightarrow \kappa_{n}$ and $\left\langle U_{n} \mid n<\omega\right\rangle$ a sequence of ultrafilters such that every condition in $S_{n}$ forces that there is a normal ultrafilter in $P_{\kappa_{n}}(\mu), F_{n}$, such that $F_{n}$ projects to $U_{n}$, and

$$
\left\{P \mid P \in P_{\kappa_{n}}(\mu) \text {, order type of } P \leq f_{n}\left(P \cap \kappa_{n}\right)\right\} \in F_{n} .
$$

Then if we define $\mathscr{P}$ as in Theorem 1 (except that we use $\operatorname{Col}\left(f\left(\alpha_{i}\right)^{+},<\kappa_{i}\right)$ instead of $\left.\operatorname{Col}\left(\alpha_{i}^{+\omega+2}<\kappa_{i}\right)\right)$, then $V^{\mathscr{P}} \vDash \Delta_{\kappa_{\omega}, \rho}$ for all regular $\kappa_{\omega}<\rho \leq \mu$.

The reason we were so particular to enumerate all our assumptions again is that we are going to apply Theorem 15 , not in $V$, but in some generic extension of $V, V^{Q}$, and so we shall have to verify that all our assumptions are satisfied. Note that if $Q$ is $\kappa_{\omega}^{+}$closed forcing and that we define $\mathscr{P}$ in $V^{Q}$ as above (using $\left\langle U_{n} \mid n<\omega\right\rangle$ and $\left\langle f_{n} \mid n<\omega\right\rangle$ ), we get that $\mathscr{P} \in V$. (This does not mean that $V^{\mathscr{P}} \vDash \Delta_{\kappa_{\omega}, \rho}$ for $\kappa_{\omega}<\rho \leq \mu$, because the assumptions about $\left\langle U_{n} \mid n<\omega\right\rangle$ and $\left\langle f_{n} \mid n<\omega\right\rangle$ are satisfied only over $V^{Q}$, so we only get $V^{Q * \mathscr{P}}=V^{\mathscr{P} \times Q} \vDash$ $\Delta_{\kappa_{\omega}, \rho}$ for $\left.\kappa_{\omega}<\rho.\right)$

\section{THE FIRST CARDINAL FIXED POINT CAN BE FULLY COMPACT}

In this section we prove

Theorem 1. Assume the consistency of infinitely many supercompacts; then there is a model of set theory in which the first cardinal fixed point (namely the first $\alpha$ such that $\alpha=\aleph_{\alpha}$ ) is fully compact (i.e., for every notion of freeness satisfying Axioms I** , II, III, IV, VI, VII, and A for a cardinal $\chi<\kappa$, if $(A, B)$ is $\kappa$ free then it is free, where $\kappa$ is the first cardinal fixed point). Also G. C. H. holds in the model. (Note that, in view of $\S 1$, we cannot have a smaller fully compact cardinal.)

The main tool for the construction will be the principle $\Delta_{\kappa, \mu}^{-}$, where for many cardinals, $\mu$, we shall get also $\Delta_{\kappa, \mu}$ to hold in our model, where $\kappa$ is the first cardinal fixed point. We do not know how to get a model in which $\Delta_{\kappa, \mu}$ holds for all regular $\mu>\kappa$ where $\kappa$ is the first cardinal fixed point (we actually suspect that it is actually false). The first cardinal for which we can construct a model in which $\Delta_{\kappa, \mu}$ holds for all regular $\mu>\kappa$, where $\kappa$ is the first cardinal fixed point of second order, namely the first $\kappa$ such that $\left\{\alpha \mid \alpha=\aleph_{\alpha}, \alpha<\kappa\right\}$ has cardinality $\kappa$. The last construction is a variation on the proof of Theorem 1 , and we shall omit it in the present paper.

As in $\S 3$ we start with a sequence of $\omega$ many supercompact $\left\langle\kappa_{n} \mid n<\omega\right\rangle$, where $\kappa_{\omega}=\sup _{n<\omega} \kappa_{n}$ G. C. H. holds above $\kappa_{\omega}$, as well as $\kappa_{n}$. We also assume that, for each $n, \kappa_{n}$ directed closed forcing does not destroy the supercompactness of $\kappa_{n}$.

Recall that $\operatorname{Col}(\alpha,<\beta)$ is the partial ordering for collapsing all cardinals strictly between $\alpha$ and $\beta$ to $\alpha$; namely it is the set of all partial functions of cardinality less than $\alpha$ whose domain is a subset of $\{\gamma \mid \gamma$ a cardinal, $\alpha<\gamma<\beta\} \times \alpha$. In particular, note that if $\beta=\delta^{+}$then $\operatorname{Col}(\alpha,<\beta)$ has 
cardinality $\delta$, and if $\beta=\alpha^{+}$then $\operatorname{Col}(\alpha,<\beta)$ is the trivial forcing notion. For regular $\alpha<\beta$ let $\operatorname{Col}^{*}(\alpha,<\beta)$ be the Easton support product of $\operatorname{Col}(\gamma,<\beta)$ for successor $\gamma, \alpha \leq \gamma<\beta$, where if there are no cardinals between $\alpha$ and $\beta$ we assume that $\operatorname{Col}^{*}(\alpha,<\beta)$ and $\operatorname{Col}(\alpha,<\beta)$ are the trivial forcing. (In the presence of G.C.H. $\mathrm{Col}^{*}(\alpha,<\beta)$ is essentially the same as $\operatorname{Col}(\alpha,<\beta)$, if $\beta$ is a successor cardinal.) The advantage of using $\operatorname{Col}^{*}(\alpha,<\beta)$ is that if $\alpha \leq \gamma<\delta \leq \beta$ then $\operatorname{Col}^{*}(\gamma,<\delta)$ is neatly embedded in $\operatorname{Col}^{*}(\alpha,<\beta)$ (namely forcing with $\operatorname{Col}^{*}(\alpha,<\beta)$ introduces a generic object for $\left.\operatorname{Col}^{*}(\gamma,<\delta)\right)$. $\operatorname{Col}^{*}(\alpha,<\beta)$ is $\alpha$ closed. If $\mathrm{G}$. C. H. holds between $\alpha$ and $\beta$, it has cardinality $\leq \beta$. If $\beta$ is regular and G.C.H. holds between $\alpha$ and $\beta$, then $\operatorname{Col}^{*}(\alpha,<\beta)$ satisfies the $\beta$ chain condition, unless $\beta$ is a successor of a singular cardinal or if $\beta$ is an inaccessible which is not Mahlo.

Let $B$ be a set of cardinals. We shall try to define a forcing notion $S(B)$ that will collapse as many cardinals as possible in the open interval $(\inf (B), \sup (B))$ while trying to preserve the cardinals in $B$. We cannot always succeed in preserving all the cardinals in $B$, the problematic cases being singular cardinals, successors of singulars, and inaccessible cardinals which are not Mahlo, so in order to have some concrete information about which cardinals are preserved we shall assume that no member of $B$ is singular, the successor of a singular, or non-Mahlo inaccessible. We also assume that if $\eta$ is a limit point of $B$ which is less than the sup of $B$ (in the sequel $\eta$ will always be singular) then $\eta^{++}$is in $B$. B satisfying all the above properties shall be called a "good" set of cardinals. If $B$ is good, let $B^{*}$ be $B$ without its last element (if $B$ has a last member). For $\delta \in B^{*}$ let $\bar{\delta}$ be the first element of $B>\delta . S(B)$ will be defined as the Easton product of $\operatorname{Col}^{*}(\delta,<\bar{\delta})$ for $\delta \in B^{*}$. Formally

$$
\begin{aligned}
& S(B)=\left\{f \mid f \text { is a partial function on } B^{*}, \text { for } \alpha \in \operatorname{Dom}(f)\right. \\
& f(\alpha) \in \operatorname{Col}^{*}(\alpha,<\bar{\alpha}) \text { and all regular } \mu, \mid f\lceil(B \cap \mu) \mid<\mu\} .
\end{aligned}
$$

$S(B)$ is clearly $\inf (B)$ directed closed. Also if $B \subseteq C$ and $C$ is good and has the same inf and sup as $B$, then forcing with $S(B)$ introduces a generic object for $S(C)$. (If $\delta \in C^{*}$ and $\bar{\delta}$ is the corresponding element of $C$, then for some $\alpha \in B, \alpha \leq \delta<\bar{\delta} \leq \bar{\alpha}$ (where $\bar{\alpha}$ is the corresponding element of $B$ and then $\operatorname{Col}^{*}(\alpha,<\alpha)$ pick a generic object for $\left.\operatorname{Col}^{*}(\delta,<\bar{\delta})\right)$. Combining these generic objects yields a generic object for $S(C)$.) If $\mu \in B$, then $S(B)$ is isomorphic to $S(B \cap(\mu+1)) \times S(B-\mu)$. Note that, in $V^{S(B)}$, every cardinal between $\inf (B)$ and $\sup (B)$ is in the closure of $B$, so there are at most $|B|$ many cardinals between $\inf (B)$ and $\sup (B)$. If G. C. H. holds between $\inf (B)$ and $\sup (B), \alpha \in B$, then $\alpha$ is still a cardinal in $V^{S(B)}$. (Here of course we use the fact that $B$ is good; namely no member of $B$ is successor of a singular or a non-Mahlo inaccessible.)

Definition 1. A set of cardinals $B$ is adequate if $B$ is $\operatorname{good}, \inf (B)=\kappa_{\omega}^{++}$, and $|B| \leq \kappa_{\omega}^{+}$; and if $\alpha$ is singular, $\alpha^{++} \in B$, and $\operatorname{cf}(\alpha) \leq \kappa_{\omega}^{+}$, then $B \cap \alpha$ is cofinal in $\alpha$.

Note that if $\lambda$ is a regular cardinal which is not a successor to a singular or 
a non-Mahlo inaccessible, $\lambda>\kappa_{\omega}$, there exists an adequate set $B$ such that $\lambda \in B$. Also the class of adequate sets is $\kappa_{\omega}^{+}$directed; namely for every subset of it of cardinality $\leq \kappa_{\omega}^{+}$, there is an adequate $B$ containing all the members of it.

Definition 2. Let $\lambda$ be a regular cardinal $\lambda>\kappa_{\omega}$. A stationary subset of $\lambda$, $S$, is called bad if, for all adequate sets $B$ with $\sup (B)>\lambda, V^{S(B)} \vDash S$ is not stationary in $\lambda$ and, for all $\alpha \in S, \operatorname{cf}(\alpha)<\kappa_{\omega}$.

Bad stationary sets cause us trouble in the construction below, so before we start our forcing construction we would like to make sure that there will be no bad stationary sets. So we shall start by iterating a forcing that destroys bad stationary sets. Note that by our assumptions bad stationary sets can occur only at the successor of a singular cardinal of cofinality less than $\kappa_{\omega}$, because if $\lambda$, which is not the successor of a singular of cofinality less than $\kappa_{\omega}, \lambda>$ $\kappa_{\omega}$, satisfies, for $\rho<\lambda, \rho^{\kappa_{\omega}}<\lambda$ (we use here G.C.H. above $\kappa_{\omega}$ ), then an argument of Baumgartner [Bal] shows that a stationary subset of $\lambda$ of points of cofinality less than $\kappa_{\omega}$ is not destroyed by a forcing notion which is $<\kappa_{\omega}$ closed. If $B$ is an adequate set of cardinals, $S(B)$ is $\kappa_{\omega}^{+}$closed. Note also that if $\lambda=\eta^{+\delta+1}$ where $\delta<\kappa_{\omega}, \eta>\kappa_{\omega}$, then there are no bad stationary subsets of $\lambda$ for the trivial reason that we can take $B=\left\{\eta^{+\rho+2} \mid \rho<\delta\right\} \cup\left\{\lambda^{++}\right\}$ and $B$ is an adequate set such that $S(B)$ is trivial, so it does not destroy any stationary subsets of $\lambda$.

It is easily seen that the definition of bad stationary subset of $\lambda$ can be formulated as follows: For every adequate $B \subseteq \lambda^{-}, B$ cofinal in $\lambda^{-}, V^{S(B)} \vDash S$ is not stationary. (By the previous remark we can consider only $\lambda$ 's which are successors of singular cardinals of cofinality $<\kappa_{\omega}$; hence $\lambda^{-}$, the predecessor of $\lambda$, is defined.) The reason is that if $S \subseteq \lambda$ is not bad, then for some adequate $C$ with $\sup (C)>\lambda, \lambda \in C, V^{S(C)} \vDash S$ is stationary. Then for every adequate $D$ containing $C$ and having the same sup, we have $V^{S(D)} \vDash S$ is stationary. (Recall that $S(C)$ neatly embeds $S(D)$.) So we can assume, without loss of generality, that $\lambda^{+}$is in $C$. Hence $C \cap \lambda^{-}$is cofinal in $\lambda^{-}$. (Recall that we are assuming that the cofinality of $\lambda^{-}$is less than $\left.\kappa_{\omega}.\right) S(C)$ is isomorphic to $S\left(C \cap\left(\lambda^{+}+1\right)\right) \times S\left(C-\lambda^{+}\right)$. Hence $V^{S\left(C \cap\left(\lambda^{+}+1\right)\right)} \vDash S$ is stationary, but since $C$ is cofinal in $\lambda^{-}, S\left(\left(C \cap \lambda^{+}+1\right)\right)=S\left(C \cap \lambda^{-}\right) . C \cap \lambda^{-}$is clearly adequate, so we have found an adequate $B \subseteq \lambda^{-}$, cofinal in $\lambda^{-}$, such that $V^{S(B)} \vDash S$ is stationary.

We are now ready to introduce a forcing notion that will kill all bad stationary sets. (Killing a stationary set means shooting a closed unbounded subset through its complement.) So let $\lambda>\kappa_{\omega}$ be a singular cardinal of cofinality $<\kappa_{\omega}$. We shall define $P^{\lambda}$ to be a forcing notion that kills all the bad stationary subsets of $\lambda^{+}$. $P^{\lambda}$ will be defined by iteration of length $\lambda^{++},\left\langle P_{\gamma}^{\lambda} \mid \gamma<\lambda^{++}\right\rangle$(we shall omit the superscript $\lambda$ because it will be fixed for a while). For limit $\gamma, P_{\gamma}$ will be the limit of $\left\langle P_{\beta} \mid \beta<\gamma\right\rangle$ with supports of size $\leq \lambda$ (namely if $\operatorname{cf}(\gamma) \leq \lambda$ we take the inverse limit; for $\operatorname{cf}(\gamma)>\lambda$ we take the direct limit). For successor 
$\lambda$, say $\gamma=\beta+1$, we pick a $P_{\gamma}$ term $\tau_{\gamma}$ forced in $V^{P_{\gamma}}$ to be a bad stationary subset of $\lambda^{+}$(if there are none, make $P_{\gamma+1}=P_{\gamma}$ ) and $R_{\gamma}$ (in $V^{P_{\gamma}}$ ) to be the forcing notion for introducing a closed unbounded subset of $\lambda^{+}$disjoint from $\tau_{\gamma}$; namely a member of $R_{\gamma}$ is a closed bounded subset of $\lambda^{+}$disjoint from $\tau_{\gamma}$. One such closed subset extends the other if it is an end extension of it. $P_{\gamma+1}$ will be $P_{\gamma} * R_{\gamma} ; P^{\lambda}$ will be $P_{\lambda^{++}}^{\lambda}$. It will follow later that $P_{\gamma}$ has a dense subset of cardinality $\leq \lambda^{+}$; hence $P^{\lambda}$ satisfies the $\lambda^{++}$chain condition, and therefore any subset of $\lambda^{+}$in $V^{P^{\lambda}}$ is already in $V^{P_{\gamma}}$ for some $\gamma<\lambda^{++}$. Also we shall prove later that $P^{\lambda}$ is distributive enough so that if a subset of $\lambda^{+}$is bad in $V^{P^{\lambda}}$, it is bad in some $V^{P_{\gamma}}$ for some $\gamma<\lambda^{++}$. So as usual we shall "dovetail" picking the terms $\tau_{\gamma}\left(\gamma<\lambda^{++}\right)$such that every bad subset of $\lambda^{+}$in $V^{P_{\gamma}}$ will be picked at some point, $\gamma$, and hence in $V^{P_{y+1}}$ it is not stationary. A forcing notion $P$ is called $\beta+1$ strategically closed if player II has a winning strategy in the game of length $\beta$, where players I and II alternate picking a smaller and smaller element of $P,\left\langle p_{\gamma} \mid \gamma<\beta\right\rangle$, where at limit stage it is player II's turn to play. Player II wins the game if $\left\langle p_{\gamma} \mid \gamma<\beta\right\rangle$ has a lower bound. (See [Sh3] for basic facts.)

Lemma 2. $P^{\lambda}$ is $\lambda+1$ strategically closed.

Proof. Since $\lambda+1$ strategically closed, forcings are closed under iteration with supports $\leq \lambda$. It is enough to show (for $\gamma<\lambda^{++}$) $V^{P_{y}} \vDash R_{\gamma}$ is strategically closed, where we also carry an induction assumption that $P_{\gamma}$ is $\lambda+1$ strategically closed. Hence $V^{P_{\gamma}}$ has the same subsets of $\lambda$ as $V$; therefore, in $V^{P_{\gamma}}, \lambda$ is still a singular cardinal of cofinality $<\kappa_{\omega}$. We now argue in $V^{P_{\gamma}}$.

Pick any adequate subset cofinal in $\lambda, B$ (we can have an adequate subset cofinal in $\lambda$ since $\left.\operatorname{cf}(\lambda)<\kappa_{\omega}\right) . \tau_{\gamma}$ is a bad subset of $\lambda^{+}$. Hence $V^{S(B)} \vDash \tau_{\gamma}$ is not stationary; hence let $\rho$ be an $S(B)$ term such that $V^{S(B)} \vDash \rho$ is a closed unbounded subset of $\lambda^{+}$disjoint from $\tau_{\gamma}$. For $\mu \in B^{*}, p \in S(B)$, let $p\lceil\mu$ be the restriction of the condition $p$ to cardinals less than or equal to $\mu$. Recall that $p$ is a function defined on $B^{*}$ so $p\left\lceil\mu\right.$ is actually $p\left\lceil B^{*} \cap(\mu+1)\right.$. Note that if $\left\langle p_{\delta}\right| \delta\langle\eta\rangle$ is a decreasing sequence of members of $S(B)$, such that, for all $\mu \in B^{*}, \mu \leq \eta, p_{\delta}\lceil\mu$ is eventually constant (below $\eta$ ), then the sequence $\left\langle p_{\delta} \mid 1 \leq \delta<\eta\right\rangle$ has a lower bound in $S(B), q$, such that, for $\mu \leq \eta, q\lceil\mu$ is eventually equal to $p_{\delta}\lceil\mu$. (Note we start indexing moves in the game by 1.)

We now describe a winning strategy for player II in the game witnessing the strategic closure of $R_{\gamma}$. Player II, besides playing the required elements in $R_{\gamma},\left\langle p_{2 \delta} \mid 1 \leq \delta<\lambda\right\rangle$, also plays (on the side) a decreasing sequence of elements of $S(B),\left\langle q_{2 \delta} \mid \delta<\lambda\right\rangle$, such that if $\delta<\delta^{\prime}$ then $q_{2 \delta}\lceil\beta(\delta)$, where $\beta(\delta)$ is the minimal member of $B^{*}$ above $\delta$. He makes sure that always $q_{2 \delta} \Vdash \sup \left(p_{2 \delta}\right) \in \rho$. Suppose we are at successor stage, player II played as his last move $p_{2 \delta}$ (and $q_{2 \delta}$ on the side), and player I responded by playing $p_{2 \delta+1} \leq p_{2 \delta}$. We have to specify player II's answer. Since $\rho$ is a term for an 
unbounded subset of $\lambda^{+}$, he can extend $q_{2 \delta}$ to $q_{2 \delta+2}$ such that, for some ordinal $\mu>\sup \left(p_{2 \delta+1}\right), q_{2 \delta+2} \Vdash \mu \in \rho$. He can even do it such that $q_{2 \delta+2}\left\lceil\beta^{*}=q_{\delta}\left\lceil\beta^{*}\right.\right.$ where $\beta^{*}$ is $\beta(2 \delta+2)$ (which is the same as $\beta(\delta)$ ). The reason he can do it is that $S(B)$ is isomorphic to $S\left(B \cap\left(\beta^{*}+1\right)\right) \times S\left(B-\beta^{*}\right)$, where the first argument in the Cartesian product is of cardinality less than $\lambda$; hence by standard arguments $V^{S(B)} \vDash\left\{\alpha \mid \alpha<\lambda^{+}\right.$, some $\left.q \in G\left(S\left(B-\beta^{*}\right)\right), q \vDash \alpha \in \rho\right\}$ is closed unbounded in $\lambda^{+}$. (By $G\left(S\left(B-\beta^{*}\right)\right)$ we mean the canonical generic filter in $S\left(B-\beta^{*}\right)$ introduced by forcing with $S(B)$.) Therefore it is enough to extend $q_{2 \delta}$ above $\beta^{*}$ to force an element of $\rho$ above $\sup \left(p_{2 \delta+1}\right)$. Player II now plays $p_{2 \delta+2}=p_{2 \delta+1} \cup\{\mu\}$. It is an element of $R_{\gamma}$ because it is a closed subset of $\lambda^{+}$and $\mu \notin \tau$. (If we had $\mu \in \tau$ we could not have a condition in $S(B)$ forcing $\mu \in \rho$ because $\rho$ was forced to be disjoint from $\tau$.)

For limit $\delta$ let $q_{\delta}$ be a lower bound of $\left\langle q_{2 \delta^{\prime}} \mid \delta^{\prime}<\delta\right\rangle$. Such a lower bound exists because if $\mu \in B^{*}, \mu<\delta$ then, for $\mu<\delta^{\prime}, \beta\left(\delta^{\prime}\right)>\mu$. Hence $q_{\delta^{\prime}}\lceil\mu$ is constant for $\delta^{\prime}>\mu$. If $\delta=\mu \in B^{*}$, then $\delta$ is a successor cardinal; hence, for large enough $\delta^{\prime}<\delta, \beta\left(\delta^{\prime}\right)=\delta$, and hence $q_{\delta^{\prime}}$ 5 is eventually constant. So by the above remarks $\left\langle q_{2 \delta^{\prime}} \mid \delta^{\prime}<\delta\right\rangle$ has a lower bound $q_{2 \delta}$ (actually $2 \delta=\delta$ ) satisfying that $q_{2 \delta}\left\lceil\mu\right.$ is eventually equal to $q_{2 \delta^{\prime}}\left\lceil\mu\right.$ for $\delta^{\prime}<\delta$ if $\mu \leq \delta$. Player II plays $q_{\delta}=\bigcup_{\delta^{\prime}<\delta} p_{2 \delta^{\prime}} \cup\left\{\sup \left(\bigcup_{\delta^{\prime}<\delta} p_{2 \delta^{\prime}}\right)\right\} ; p_{\delta}$ is clearly closed (each $p_{2 \delta^{\prime}}$ end extended the previous $p_{2 \delta^{\prime}}$ ). The only problem in showing $p_{\delta} \in R_{\gamma}$ is to show that $\alpha=\sup \left(\bigcup_{\delta^{\prime}<\delta} p_{2 \delta^{\prime}}\right) \notin \tau$, but, for each $\delta^{\prime}<\delta, q_{2 \delta^{\prime}} \Vdash \sup \left(p_{2 \delta^{\prime}}\right) \in \rho$, and hence $q_{2 \delta} \Vdash \sup \left(p_{2 \delta^{\prime}}\right) \in \rho$. Since $\rho$ is forced to be closed,

$$
q_{2 \delta} \Vdash \alpha=\sup \left(\left\langle\sup \left(p_{2 \delta^{\prime}}\right) \mid \delta^{\prime}<\delta\right\rangle\right) \in \rho .
$$

Hence we must have $\alpha \notin \tau$. A similar argument shows that if player II was following this strategy through $\lambda$ many steps, then $\left\langle p_{\delta} \mid \delta<\lambda\right\rangle$ has a lower bound.

Note that in Lemma 2 we did not use any special properties of $V$ except the G. C. H. above $\kappa_{\omega}$. So it holds in appropriate forcing extensions of $V$.

Lemma 3. $P^{\lambda}$ satisfies $\lambda^{++}$-c.c.

Proof. $P^{\lambda}$ is the direct limit of the $P_{\gamma}$ for $\gamma<\lambda^{++}$where, for $\gamma$ of cofinality $\lambda^{+}, P_{\gamma}$ is the direct limit of $P_{\delta}$ for $\delta<\gamma$. By a standard argument it is enough to show that $P_{\gamma}$ satisfies $\lambda^{++}$c.c. This will follow easily if we show that $P_{\gamma}$ has a dense subset of cardinality $\lambda^{+}$. A member of $P_{\gamma}$ can be considered to be a sequence $\left\langle\eta_{\delta} \mid \delta<\gamma\right\rangle$, where, for $\delta<\gamma, \eta_{\delta}$ is a term forced by every condition in $P_{\delta}$ to be a member of $R_{\delta}$. Also the set $\left\{\delta<\gamma \mid \eta_{\delta} \neq \varnothing\right\}$ has cardinality $\leq \lambda$. By Lemma $2, P_{\delta}$ is $\lambda+1$ strategically closed, $P_{\delta}$ introduces no new bounded subsets of $\lambda^{+}$, and hence every member of $R_{\delta}$ is in $V$. (We are not claiming that $R_{\delta}$ is in $V$.) Let $P_{\gamma}^{*}$ be the set of all members of $P_{\gamma},\left\langle\eta_{\delta} \mid \delta<\gamma\right\rangle$, such that, for all $\delta<\gamma, \eta_{\delta}=\hat{c}_{\delta}$ for some $c_{\delta} \in V . c_{\delta}$ is a bounded subset of $\lambda^{+}$, so using G.C.H. we can show that the cardinality of $P_{\gamma}^{*}$ is $\lambda^{+}$. Using Lemma 2 again we can show that $P_{\gamma}^{*}$ is dense in $P_{\gamma}$. 
It follows from Lemma 3 that every subset of $\lambda^{+}$in $V^{P^{\lambda}}$ is already in $V^{P_{\gamma}}$ for some $\gamma<\lambda^{++}$. Note also that in view of Lemma 2, forcing with $P^{\lambda}$ does not change the collection of adequate subsets of $\lambda$ or the definition of $S(B)$ for an adequate $B \subseteq \lambda$. Hence if $\tau$ is a bad subset in $V^{P^{\lambda}}$, we can find $\delta<\lambda^{++}$ such that $\mu$ is already bad in $V^{P_{\gamma}}$ for every $\gamma \geq \delta$. (Pick $\delta$ large enough so that $\tau \in V^{P_{\delta}}$.) Also make sure that, for every adequate $B \subseteq \lambda$, in $V^{P_{\delta}}$ we can find an $S(B)$ term, forced by $S(B)$ to be a closed unbounded subset of $\lambda^{+}$ disjoint from $\tau$. We can find such a $\delta$ because there are at most $\lambda^{+}$adequate $B \subseteq \lambda$ and an $S(B)$ term for a subset of $\lambda^{+}, \eta(B)$, can be coded by a subset of $\lambda^{+}$; hence it belongs to some $V^{P_{\delta}}$ for $\delta<\lambda^{++}$large enough. (We can also verify that once this term belongs to $V^{P_{\delta}}$; we have that $V^{P_{\delta}} \vDash S(B) \Vdash$ “ $\eta(B)$ is a closed unbounded subset of $\lambda^{+}$disjoint from $\tau$.") The above remarks show that we can pick the terms $\tau_{\gamma}$ for $\gamma<\lambda^{++}$such that every $\tau \in V^{P^{\lambda}}$ which is a bad subset of $\lambda^{+}$will be the realization of some $\tau_{\gamma}$, and hence it will be nonstationary in $V^{P^{\lambda}}$. We have proved

Lemma 4. Under appropriate choice of the terms $\tau_{\gamma}$ for $\gamma<\lambda^{++}$,

$$
V^{P^{\lambda}} \vDash \text { there are no bad stationary subsets of } \lambda^{+} \text {. }
$$

Now we are going to iterate the forcing notions $P^{\lambda}$ through all the singular cardinals $\lambda>\kappa_{\omega}$. So the steps of the iteration will be denoted by $Q_{\alpha}(\alpha$ an ordinal), where $Q_{0}$ is the trivial forcing notion, and if $\alpha$ is not a singular cardinal bigger than $\kappa_{\omega}$ or $\operatorname{cf}(\alpha)>\kappa_{\omega}, Q_{\alpha+1}=Q_{\alpha} *$ (the trivial forcing). The only case in which we do something interesting is when $\alpha$ is a singular cardinal greater than $\kappa_{\omega}$ but whose cofinality is less than $\kappa_{\omega}$. In this case $Q_{\alpha+1}=Q_{\alpha} * P^{\alpha} \quad\left(P^{\alpha}\right.$ is of course taken in the sense of $\left.V^{Q_{\alpha}}\right)$. At limit $\alpha$ we take $Q_{\alpha}$ to be the inverse limit of $\left\langle Q_{\beta} \mid \beta<\alpha\right\rangle$, where for regular $\alpha$ we take $Q_{\alpha}$ to be the nonstationary limit; namely the support must be a nonstationary subset of $\alpha$. Note that this assumption is meaningful only for inaccessible $\alpha$ since, for successor $\alpha$, say $\alpha=\beta^{+}$, we iterate the trivial forcing between $\beta$ and $\alpha$. The final forcing we shall use is $Q=\cup Q_{\beta}$, which is a class forcing. Note that, for each singular $\lambda, Q=Q_{\lambda} * P^{\lambda} *$ (the iteration of $P^{\lambda^{\prime}}$ for $\lambda^{\prime}>\lambda$ ). This last iteration will be denoted by $Q_{\lambda}^{\infty}$. (The iteration between $\alpha$ and $\beta$ will be denoted by $Q_{\alpha}^{\beta}$.) $P^{\lambda^{\prime}}$ for $\lambda^{\prime}>\lambda$ is by Lemma 4 (applied in $V^{Q_{\lambda}}$ ) a $\lambda^{+3}$ strategically closed forcing, and hence $Q_{\lambda}^{\infty}$ is $\lambda^{+3}$ strategically closed. (We use the fact that we were using inverse limits at singulars.) This can be used, by the usual arguments, to show that $V^{Q}$ is a model of set theory (see for instance Jech's book [Je] where we replace the completeness argument by strategic closure). We can use it to show that $V^{Q} \vDash$ G. C. H. above $\kappa_{\omega}$. Also all subsets of $\lambda^{+}$in $V^{Q}$ are already in $V^{Q_{\lambda+1}}$. So $S \subseteq \lambda^{+}$is bad in $V^{Q}$ iff it is bad in $V^{Q_{\lambda+1}}=\left(V^{Q_{\lambda}}\right)^{P^{\lambda}} \quad\left(P^{\lambda}\right.$ is taken in the sense of $\left.V^{Q_{\lambda}}\right)$. Using Lemma 4 in $V^{Q_{i}}$ we get that in $V^{Q}$ there are no bad stationary sets. 
From now on our ground model is going to be $V^{Q}$. Our main problem now is that the $\kappa_{n}$ for $n<\omega$ are probably not supercompact. The following lemma will allow us to recover some of their supercompactness.

Lemma 5. Let $\lambda$ be a singular cardinal, $B$ adequate such that $\lambda=\sup (B)$; then $Q_{\lambda+1} * S(B)$ is essentially $\mu=\kappa_{\omega}^{+}$directed closed (where a forcing notion is said to be essentially $\mu$-directed closed if it has a dense subset which is $\mu$-directed closed).

Note that $S(B)$ is taken in the sense of $V^{Q_{\lambda+1}}$, but it is the same as $S(B)$ in the sense of $V^{Q}$ or of $V^{Q_{\lambda}}$ (since $P^{\lambda}$ does not introduce new subsets of $\lambda$ ). The lemma is easy for the $\lambda$ which is the first cardinal such that $\lambda^{+}$carries a bad stationary set, because by forcing with $S(B)$ first (it is the same in $V$ and $V^{Q_{\lambda+1}}$ ) we get that all the "stationary" sets we try to kill are already killed by $S(B)$, so $Q_{\lambda+1}$ is a $<\lambda^{+}$closed forcing, so the lemma follows in this case. This is the main idea behind the proof of the lemma, except that in the general case we need much more elaborate bookkeeping of portions of $S(B)$ 's (taken in different models of set theory).

Proof. Recall that in the definition of $P^{\gamma}$ (as an iteration of the length $\gamma^{++}$) we used a sequence of terms $\left\langle\tau_{\mu}^{\gamma} \mid \mu<\gamma^{++}\right\rangle\left(\tau_{\mu}^{\gamma}\right.$ was essentially a $Q_{\gamma} * P_{\mu}^{\gamma}$ term for a bad subset of $\gamma^{+}$). Since $\tau_{\mu}^{\gamma}$ is bad it is destroyed by any $S(C)$ where $C \subseteq \gamma, C$ adequate, and $C$ cofinal in $\gamma$. For every $\gamma$ (limit singular cardinal of cofinality $\left.<\kappa_{\omega}\right), \gamma \leq \lambda$, we shall define $C_{\gamma} \subseteq \gamma, C_{\gamma}$ adequate and cofinal in $\gamma$, and a neat embedding of $S\left(C_{\gamma}\right)$ into $S(B)$ (namely a way of getting a generic filter of $S\left(C_{\gamma}\right)$ from one for $\left.S(B)\right)$. Since $S\left(C_{\gamma}\right)$ in the sense of $V^{Q_{\lambda}}$ is the same as in the sense of $V^{Q_{\gamma}}$, which is the same as $S\left(C_{\gamma}\right)$ in the sense of $V^{Q_{\gamma}^{*} P_{\mu}^{\gamma}}\left(\mu<\gamma^{++}\right)$,

$$
V^{Q_{\gamma} * P_{\mu}^{\gamma} * S\left(C_{\gamma}\right)} \vDash \tau_{\mu}^{\gamma} \text { is not stationary. }
$$

Therefore in $V^{Q_{\gamma} * P_{\mu}^{\gamma}}$ there is an $S\left(C_{\gamma}\right)$ term $\rho_{\mu}^{\gamma}$ such that it is forced by $S\left(C_{\gamma}\right)$ that $\rho_{\mu}^{\gamma}$ is a closed unbounded subset of $\gamma^{+}$disjoint from $\tau_{\mu}^{\gamma}$. Since we will have an embedding of $S\left(C_{\gamma}\right)$ into $S(B)$, we can consider $\rho_{\mu}^{\gamma}$ to be an $S(B)$ term (in $V^{Q_{\lambda+1}}$ ) denoting, of course, a closed unbounded subset of $\gamma^{+}$.

$C_{\gamma}$ will be defined as follows. If $B \cap \gamma$ is cofinal in $\gamma$, then $C_{\gamma}=(B \cap \gamma)$; otherwise, $B \cap \gamma$ is bounded in $\gamma$ and by $B$ being good $B \cap \gamma$ has a maximal element $\mu$. In this case we put $C_{\gamma}=(B \cap \gamma) \cup D_{\gamma}$, where $D_{\gamma}$ is an adequate unbounded subset of $\gamma$ whose minimum is above $\mu$. (We use the fact that $\left.\operatorname{cf}(\gamma)<\kappa_{\omega}.\right)$ In case $B \cap \gamma$ is unbounded in $\gamma$, there is a natural way to embed $S\left(C_{\gamma}\right)$ into $S(B)$ (for $f \in S(B), f\left\lceil(B \cap \gamma)\right.$ is in $S\left(C_{\gamma}\right)$ ). In case $B \cap \gamma$ is bounded in $\gamma$, we identify a condition $f \in S(C)$ with $f^{*}$ in $S(B)$ as follows. Let $\mu$ be $\sup (B \cap \gamma)$. The domain of $f^{*}$ will be (domain $\left.(f) \cap B\right) \cup\{\mu\}$. If $\delta \in C, \delta<\mu$, then $\delta \in B$ and we put $f^{*}(\delta)=f(\delta)$. (Note that $f^{*}(\delta)$ belongs to the right set, namely $\operatorname{Col}^{*}(\delta,<\bar{\delta})$, because $\bar{\delta}$ in the sense of $B$ is the same as $\bar{\delta}$ in the sense of $C$.) For $\delta=\mu$ we want to define $f^{*}(\mu)$ as 
a partial function in $\times_{\mu \leq \rho<\bar{\mu}} \operatorname{Col}(\rho,<\bar{\mu})$ where $\bar{\mu}$ is the next member of $B$ above $\mu . f^{*}(\mu)(\rho)$ will be defined only if $\rho$ is a successor cardinal between $\mu$ and $\gamma$ such that, for some $\eta \in C_{\gamma}, \eta \leq \rho<\bar{\eta}$, where $\bar{\eta}$ is the minimal member of $C_{\gamma}$ above $\eta$. In this case we put $f^{*}(\mu)(\rho)=f(\eta)(\rho)$. Note that $f^{*}(\mu)(\rho)$ belongs to the right set, since it belongs to $\operatorname{Col}(\rho,<\bar{\eta})$ which can be considered to be a subset of $\operatorname{Col}(\rho,<\bar{\mu})$, since $\bar{\eta}<\bar{\mu}$. It can be easily checked that the map we defined is a neat embedding of $S\left(C_{\gamma}\right)$ into $S(B)$.

For $f \in S(B), \gamma \leq \lambda, \gamma$ a limit cardinal, we define $f\lceil\gamma$ as follows (we are not assuming $\gamma \in B)$. If $B \cap \gamma$ is unbounded in $\gamma$, then $f\left\lceil\gamma\right.$ is $f\left\lceil\left(B^{*} \cap \gamma\right)\right.$. If it is bounded and $\mu=\sup (B \cap \gamma)$, then $f\left\lceil\gamma\right.$ is $f\left\lceil(B \cap \mu) \cup\left\{\left\langle\mu, f^{*}(\mu)\right\rangle\right\}\right.$ where $f^{*}(\mu)(\rho)=f(\mu)(\rho)$ if $\rho<\gamma$, and is undefined otherwise. $f\lceil\gamma$ is essentially the information $f$ gives about collapses where the target cardinals are below $\gamma$. Note that if $\left\langle f_{\alpha} \mid \alpha<\rho\right\rangle(\rho \leq \gamma)$ is a decreasing sequence of elements of $S(B)$ such that, for all $\mu<\gamma, f_{\alpha}\lceil\mu$ is eventually constant, then this sequence has a lower bound in $S(B)$. Also a decreasing sequence of length $\leq \mu \quad(\mu$ limit cardinal), with $f_{\alpha}\lceil\mu$ constant, has a lower bound.

$S(B)\lceil\gamma$ will be $\{f\lceil\gamma \mid f \in S(B)\}$. Of course, forcing with $S(B)$, we introduce a generic filter to $S(B)\left\lceil\gamma\right.$. If $C_{\gamma}$ is defined as above and $S\left(C_{\gamma}\right)$ embedded as above into $S(B)$, then actually $V^{S\left(C_{\gamma}\right)} \subseteq V^{S(B)\lceil\gamma}$. Also $S(B)\left\lceil\gamma \in V^{Q_{y}}\right.$ (because $Q_{\gamma}^{\lambda+1}$ is $\gamma$ distributive and any element of $S(B)\lceil\gamma$ can be coded as a sequence of $\gamma$ ordinals).

The elements of $Q_{\lambda+1} * S(B)$ are of the form $(q, \tau)$ where $\tau$ is a $Q_{\lambda+1}$ term for a member of $S(B)$. We would like to use the fact that, for $\gamma<\lambda, \tau\lceil\gamma$ is in $V^{Q_{\nu}}$, to get a simpler form of terms.

Definition 3. (a) A $Q_{\lambda+1}$ term, $\tau$, forced to denote an element of $S(B)$ is called canonical if for every limit cardinal $\gamma, \gamma \leq \lambda, \tau\left\lceil\gamma\right.$ is essentially a $Q_{\gamma}$ term. Namely whether $x \in V^{Q_{\gamma}}$ is in $\tau\lceil\gamma$ or not depends only on conditions in $Q_{\gamma}$. More formally if $q \in Q_{\lambda}, x$ a $Q_{\gamma}$ term, $q \Vdash x \in \tau\lceil\gamma$ iff $q\lceil\gamma \Vdash x \in \tau\lceil\gamma$, and similarly for the statement " $x \notin \tau\left\lceil\gamma\right.$ ". (Recall that members of $Q_{\lambda}$ are functions, $q$, where $q(\gamma)$ is a $Q_{\gamma}$ term for an element of $P^{\gamma}$ so $q\lceil\gamma$ makes sense.)

(b) A term as in (a) is called $\chi$ canonical $(\chi \leq \lambda)$ if (a) holds for $\chi \leq \gamma$.

Note that if $\gamma$ is a limit cardinal and, for all $\beta<\gamma, \tau\left\lceil\beta\right.$ is a $Q_{\beta}$ term, then $\tau\left\lceil\gamma\right.$ is a $Q_{\gamma}$ term.

Lemma 6. Let $(q, \tau) \in Q_{\lambda} * S(B)$; then there exists a canonical $\tau^{\prime}$ and $q^{\prime} \leq q$ such that $q^{\prime} \Vdash \tau^{\prime}=\tau$.

Proof. $Q_{\lambda+1}$ is obtained by iteration of forcings, which is either a trivial forcing notion or is of the form $P^{\gamma}$. In either case at the $\gamma$ th stage we use a forcing which is $\gamma+1$ strategically closed. So for each $\gamma \leq \lambda=1$ let $F_{\gamma}$ be a $Q_{\gamma}$ term for a winning strategy for player II in the game of length $\gamma+1$ in $P^{\gamma}$ (where $P^{\gamma}$ can be the trivial forcing notion in case $\gamma$ is not a limit cardinal $>\kappa_{\omega}$ of cofinality $<\kappa_{\omega}$ or there are no bad stationary subsets of $\left.\gamma^{+}\right) . F_{\gamma}$ 
applies to decreasing sequences of elements of $P^{\gamma}$ of length $\leq \gamma$ and gives II's response in case that the previous play is the given sequence. We define $F_{\gamma}$ on a sequence which is not a legitimate play of the game or a sequence in which player II was not following his strategy arbitrarily. We can assume without loss of generality that the strategy is such that as long as I plays the trivial condition, II's responses are trivial.

Given a decreasing sequence of conditions in $Q_{\lambda+1},\left\langle q_{\alpha} \mid \alpha<\gamma\right\rangle$, we define our master strategy as follows: $F\left(\left\langle q_{\alpha} \mid 0<\alpha<\gamma\right\rangle\right)=q^{*}$ where, for $\rho<\gamma, q^{*}(\rho)$ is some term forced to be below $\left\langle q_{\alpha}(\rho) \mid 0<\alpha<\gamma\right\rangle$ if such a lower bound exists and the trivial condition otherwise. (In case $q_{\alpha}(\rho)$ is eventually constant we define $q^{*}(\rho)$ to be this constant.) For $\gamma \leq \rho, q^{*}(\rho)$ is the $Q_{\rho}$ term denoting $F_{\rho}\left(\left\langle q_{\alpha}(\rho) \mid 0<\alpha<\gamma\right\rangle\right) . q^{*}$ is not clearly in $Q_{\rho}$ because it does not necessarily have the right support, but we have the following:

Claim. Let $\left\langle q_{\alpha} \mid 0<\alpha<\gamma\right\rangle$ be a decreasing sequence of elements of $Q_{\lambda+1}$ where, for $\delta<\gamma, q_{\alpha}(\delta)$ is constant for $\alpha>\delta$. Also the set $\left\{\delta \mid q_{\alpha}(\delta)\right.$ is nontrivial for some $\alpha$ but for $\alpha<\delta$ it is trivial $\}$ is not stationary in any regular cardinal $\mu$. For $\gamma \leq \delta$ we assume $q\left\lceil\delta \Vdash\right.$ "The sequence $\left\langle q_{\alpha}(\delta) \mid 0<\alpha<\gamma\right\rangle$ is an initial segment of a play of the game for $P^{\delta}$ in which the strategy $F_{\delta}$ was followed". Then $q^{*}=F\left(\left\langle q_{\alpha} \mid 0<\alpha<\gamma\right\rangle\right)$ is in $Q_{\lambda+1}$, it is below each $q_{\alpha}(\alpha<\gamma)$, and for each $\gamma \leq \delta, q^{*}\left\lceil\delta \Vdash\left\langle q_{\alpha}(\delta) \mid 0<\alpha<\gamma\right\rangle^{\wedge}\left\langle q^{*}(\delta)\right\rangle\right.$ is an initial segment of the play of the game for $P^{\delta}$ in which the strategy $F_{\delta}$ was followed.

Proof. The proof of the claim is rather obvious. The only point that should be elaborated on is to show that, for all regular $\mu,\left\{\delta \mid \delta<\mu, q^{*}(\delta)\right.$ is nontrivial $\}$ is nonstationary in $\mu$. Assume that $A=\left\{\delta \mid \delta<\mu, q^{*}(\delta)\right.$ is nontrivial $\}$ is stationary in $\mu$. Note that if $q^{*}(\delta)$ is nontrivial there is $\alpha(\delta)<\delta$ such that $q_{\alpha(\delta)}(\delta)$ is nontrivial. By our assumption, except for nonstationarily many $\delta<$ $\mu$, we can assume $\alpha(\delta)<\delta$ but then $\alpha(\delta)$ is essentially a pressing down function on $A$, hence it is constant on a stationary subset of $A, A^{*}$, but if $\alpha$ is this constant value it means that $A^{*} \subseteq\left\{\delta \mid \delta<\mu, q_{\alpha}(\delta)\right.$ is nontrivial $\}$, which is a contradiction to $q_{\alpha} \in Q_{\lambda+1}$.

We resume the proof of Lemma 6 . We define by induction a decreasing sequence of elements of $Q_{\lambda+1}$ of length $\lambda+1,\left\langle q_{\alpha} \mid 0<\alpha<\lambda+1\right\rangle$, and a sequence of terms $\tau_{\alpha}, \alpha<\lambda, \alpha$ a limit cardinal such that $\tau_{\alpha}$ is a $Q_{\alpha}$ term and $q_{\alpha+1} \Vdash \tau\left\lceil\alpha=\tau_{\alpha} \cdot q_{0}\right.$ will be our given $q$. For even ordinal $\alpha$ define $q_{\alpha+1}$ to be $q_{\alpha}$, unless $\alpha$ is a limit cardinal, in which case we know that $\tau\lceil\alpha$ is forced to be in $V^{Q_{\alpha}}$. Hence there is an extension of $q_{\alpha}, q_{\alpha+1}$, and a $Q_{\alpha}$ term $\tau_{\alpha}$ such that $q_{\alpha+1}\left\lceil\alpha=q\left\lceil\alpha\right.\right.$ and $q_{\alpha+1} \Vdash \tau\left\lceil\alpha=\tau_{\alpha}\right.$. If possible we pick $q_{\alpha+1}=q_{\alpha}$. (Note that if $\alpha$ is a limit cardinal which is a limit of limit cardinals, then we can pick $q_{\alpha+1}=q_{\alpha}$ because we can find a $Q_{\alpha}$ term $\tau_{\alpha}$ such that for $\beta<\alpha$ it is forced by $q_{\alpha}, \tau_{\alpha}\left\lceil\beta=\tau_{\beta}.\right)$ For odd $\alpha$ let $q_{\alpha+1}=F\left(\left\langle q_{\beta} \mid 0<\beta \leq \alpha\right\rangle\right)$. For limit $\alpha, q_{\alpha}=F\left(\left\langle q_{\beta} \mid 0<\beta<\alpha\right\rangle\right)$.

By induction it is easy to see that, for $\gamma \leq \lambda,\left\langle q_{\alpha} \mid \alpha<\gamma\right\rangle$ satisfies all the requirements of the claim and that, for $\alpha<\alpha^{\prime}, q_{\alpha^{\prime}}\left\lceil\alpha=q_{\alpha}\lceil\alpha\right.$. (We use the fact 
that $\alpha$ is a limit and $q_{\beta}(\alpha)$ is trivial for $\beta<\alpha$; then $q_{\alpha}(\alpha)$ is trivial. The only way we can get $q_{\alpha+1}(\alpha)$ nontrivial while $q_{\alpha}(\alpha)$ is trivial is when $\alpha$ is a limit cardinal which is not the limit of limit cardinals but the set $\{\alpha \mid \alpha \leq \lambda, \alpha$ a limit cardinal but not limit of limit cardinals $\}$ is never stationary.) Thus using the claim we show that $\left\langle q_{\gamma} \mid \gamma \leq \lambda\right\rangle$ are really decreasing. Now we can easily define a term $\tau^{\prime}$ such that $\tau^{\prime}\left\lceil\alpha=\tau_{\alpha}\right.$. Clearly $\tau^{\prime}$ is canonical and $q_{\lambda} \Vdash \tau^{\prime}=\tau$.

We resume the proof of Lemma 5 , and at last we are ready to define the dense set of elements of $Q_{\lambda+1} * S(B)$, which will be $\kappa_{\omega}^{+}$closed.

This set, to be denoted by $R$, is the set of all elements of $Q_{\lambda+1} * S(B)$ of the form $(q, \tau)$ where $\tau$ is canonical and for every limit cardinal $\gamma$ for which $P^{\gamma}$ is not trivial:

$$
\begin{aligned}
& q\left\lceil\gamma \Vdash " \forall \delta \delta<\gamma^{++} \text {If } q(\gamma)(\delta)\right. \text { is not trivial, then } \\
& \qquad q(\gamma)\left\lceil\delta \Vdash _ { P _ { \delta } ^ { \gamma } } \tau \left\lceil\gamma \Vdash_{S(B)\lceil\gamma} \sup q(\gamma)(\delta) \in \rho_{\delta}^{\gamma^{\prime}} .\right.\right.
\end{aligned}
$$

Explanation. $q(\gamma)$ is a $Q_{\gamma}$ term denoting an element of $P^{\gamma}$, and $q(\gamma)(\delta)$ is a $P_{\delta}^{\gamma}$ term denoting an initial segment of the closed unbounded subset of $\gamma^{+}$ we are trying to force in the complement of $\tau_{\delta}^{\gamma}$. Since $\tau$ is canonical, $\tau\lceil\gamma$ is essentially a $Q_{\gamma}$ term for an element of $S(B)\left\lceil\gamma . S(B)\left\lceil\gamma\right.\right.$ (via $S\left(C_{\gamma}\right)$, which it embeds) introduces a closed unbounded subset of $\gamma^{+}$, disjoint from $\tau_{\delta}^{\gamma}$, when we force over $\left(V^{Q_{\gamma}}\right)^{P_{\delta}^{\gamma}}$. We denoted this set by $\rho_{\delta}^{\gamma}$. So our requirement is that the information we have about the generic filters on $Q_{\gamma}, P_{\delta}^{\gamma}$, and $S(B)\lceil\gamma$ will force the sup of the initial segment of the set avoiding $\tau_{\delta}^{\gamma}$ to be in $\rho_{\delta}^{\gamma}$.

The fact that $R$ is the required subset of $Q_{\lambda+1} * S(B)$ naturally breaks into two sublemmas.

Sublemma 7. $R$ is $\kappa_{\omega}^{+}$-directed closed.

Proof. Let $D \subseteq R$ be a directed set. $|D| \leq \kappa_{\omega}$. We want to show that $D$ has a lower bound in $R$. Let $D^{*}=\left\{q \mid q \in Q_{\lambda+1}\right.$, for some $\left.\tau,(q, \tau) \in D\right\}$ and $D^{* *}=\left\{\tau \mid\right.$ for some $\left.q \in Q_{\lambda+1},(q, \tau) \in D\right\}$. Clearly $D^{*}$ is easily seen to be directed; also if we manage to get a lower bound for $D^{*}, q^{*}$ (which we shall do in a minute), then $q^{*} \Vdash D^{* *}$ (as a subset of $S(B)$ ) is directed. We denote by $D^{* *}$ both the set of terms in $V$ and the set of their realizations in $V^{Q_{\lambda+1}}$.

We now define $q^{*}$, which is assumed to be a lower bound for $D^{*}$. Of course if $\gamma \leq \lambda$ is such that $P^{\gamma}$ is trivial, then $q(\gamma)$ is trivial. If $P^{\gamma}$ is nontrivial, then $q^{*}(\gamma)(\delta) \quad\left(\delta<\gamma^{++}\right)$is defined to be a $Q_{\gamma} * P_{\delta}^{\gamma}$ term denoting the union of $\{q(\gamma)(\delta) \mid q \in D\}$, together with its sup. For any $\delta$ for which $q^{*}(\gamma)(\delta)$ is nontrivial, $q^{*}(\delta)$ is the union of at most $\kappa_{\omega}$ sets of cardinality $\leq \gamma$; hence it is of cardinality $\leq \gamma$.

Now we prove by induction on $\gamma \leq \lambda+1$ that $q^{*}\left\lceil\gamma \in Q_{\gamma}\right.$, and that

$$
q^{*}\left\lceil\gamma \Vdash q^{*}(\gamma) \in P^{\gamma}\right.
$$

and

$$
q^{*}\left\lceil\gamma \Vdash q^{*}(\gamma) \text { is a lower bound for }\left\{q(\gamma) \mid q \in D^{*}\right\}=D_{\gamma}^{*}\right. \text {. }
$$


$\left(D_{\gamma}^{*}\right.$ is a set in $V^{Q_{\gamma}}$, where we assume $q^{*}\left\lceil\gamma \in G\left(Q_{\gamma}\right)\right.$.) The only interesting case of the induction is when $P^{\gamma}$ is nontrivial. (In particular $\gamma$ is a limit cardinal $>\kappa_{\omega}$.) The fact that $q^{*}\left\lceil\gamma \in Q_{\gamma}\right.$ follows easily from the inductive assumption, since for $\gamma^{\prime}<\gamma, q^{*}\left\lceil\gamma^{\prime} \Vdash q^{*}\left(\gamma^{\prime}\right) \in P^{\gamma^{\prime}}\right.$, and if $\mu$ is regular, $\kappa_{\omega}<\mu<\gamma$, then the support of $q^{*}\left\lceil\gamma\right.$ is in $\mu$ is the union of $\kappa_{\omega}$ supports of $q \in D$; hence it is the union of less than $\mu$ nonstationary subsets of $\mu$, and therefore it is a nonstationary subset of $\mu$. (Note that if $\mu<\kappa_{\omega}$ then the forcing up to $\mu$ is trivial.) Also the inductive assumption gives that $q^{*}\lceil\gamma$ extends $\left\{q\left\lceil\gamma \mid q \in D^{*}\right\}\right.$. Since $D$ is directed it follows that $q^{*}\left\lceil\gamma \Vdash D_{\gamma}\right.$ is directed. By a remark above, the support of $q^{*}(\gamma)$ is of cardinality $\leq \gamma$. In order to simplify notation assume that we argue over $V^{Q_{\gamma}}$, where $q^{*}\left\lceil\gamma \in G\left(Q_{\gamma}\right)\right.$. (It will then follow that whatever we prove about $V^{Q_{\gamma}}$ will be forced by $q^{*}\lceil\gamma$.)

By induction on $\delta<\gamma^{++}$we show that $q^{*}(\gamma)\left\lceil\delta \in P_{\delta}^{\gamma}\right.$. The crucial inductive step is that

$$
q^{*}(\gamma)\left\lceil\delta \Vdash_{P_{\delta}^{\gamma}} q^{*}(\gamma)(\delta) \text { is a closed bounded subset disjoint from } \tau_{\delta}^{\gamma} .\right.
$$

We also need by induction that $q^{*}(\gamma)\left\lceil\delta\right.$ is below $\left\{q(\gamma)\left\lceil q \in D^{*}\right\}\right.$. If we have it for $\delta$, then $D_{\gamma, \delta}^{*}=\left\{q(\gamma)(\delta) \mid q \in D^{*}\right\}$ is forced by $q^{*}(\gamma)\lceil\delta$ to be a directed subset of the forcing shooting a closed unbounded subset through the complement of $\tau_{\delta}^{\gamma} \cdot q^{*}(\gamma)(\delta)$ is clearly forced by $q^{*}(\gamma)\lceil\delta$ to be a closed bounded subset of $\gamma^{+}$which extends each element in $D_{\gamma, \delta}^{*}$. The only problem is to show that it is forced to be disjoint from $\tau_{\delta}^{\gamma}$, and the only point which is problematic is the sup of $q^{*}(\gamma)(\delta)$, which we denote by $\mu$. So we have to show that $q^{*}(\gamma)\left\lceil\delta \Vdash_{P_{\delta}^{\gamma}} \mu \notin \tau_{\delta}^{\gamma}\right.$.

The fact that $q^{*}\left\lceil\gamma\right.$ is below $\left\{q\left\lceil\gamma \mid q \in D^{*}\right\}\right.$ implies that $\left\{\tau\left\lceil\gamma \mid \tau \in D^{* *}\right\}\right.$ is forced by $q^{*}\left\lceil\gamma\right.$ to be a directed subset of $S(B)\left\lceil\gamma\right.$. (Each $\tau \in D^{* *}$ is canonical; therefore $\tau\left\lceil\gamma\right.$ is a $Q_{\gamma}$ term.) Since $S(B)\left\lceil\gamma\right.$ is forced to be $\kappa_{\omega}^{+}$directed closed, there is a $Q_{\gamma}$ term $\tau_{\gamma}^{*}$ forced by $q^{*}\lceil\gamma$ to be the maximal lower bound to $\left\{\tau\left\lceil\gamma \mid \tau \in D^{* *}\right\} . \tau_{\gamma}^{*}\right.$ extends each $\tau\left\lceil\gamma\right.$ for $\tau \in D^{* *}$; therefore by definition of $R$

$$
q^{*}\left\lceil\gamma \Vdash _ { Q _ { \gamma } } q ^ { * } ( \gamma ) \left\lceil\delta \Vdash_{P_{\delta}^{\gamma}} \tau_{\gamma}^{*} \Vdash_{S(B)\lceil\gamma}\left\{\sup (q(\gamma)(\delta)) \mid q \in D^{*}\right\} \subseteq \rho_{\delta}^{\gamma} .\right.\right.
$$

Since $\rho_{\delta}^{\gamma}$ is forced to be a closed unbounded subset of $\gamma^{+}$, we get in $\left(V^{Q_{y}}\right)$

$$
q^{*}(\gamma)\left\lceil\delta \Vdash \tau_{\gamma}^{*} \Vdash_{S(B)\lceil\gamma} \sup \left\{\sup (q(\gamma)(\delta)) \mid q \in D^{*}\right\} \in \rho_{\delta}^{\gamma},\right.
$$

but this last sup is really $\mu$. Now $\rho_{\delta}^{\gamma}$ is supposed to be disjoint from $\tau_{\delta}^{\gamma}$. If in $V^{Q_{\gamma} * P_{\delta}^{\gamma}}$ some condition is $S(B)\left\lceil\gamma\right.$ forces $\mu$ to be in $\rho_{\delta}^{\gamma}$, we must have that $\mu \notin \tau_{\delta}^{\gamma}$, which concludes the proof that $q^{*}(\gamma) \in P^{\gamma}$.

We have shown that $q^{*}$ is in $Q_{\lambda+1}$, and it is below all the conditions in $D^{*}$. So $D^{* *}$ is directed in $S(B)$, and hence it has a least upper bound, which we denote by $\tau^{*}$. If one recalls the definition of $\tau_{\gamma}^{*}$ above, one sees that $q^{*} \Vdash \tau^{*}\left\lceil\gamma=\tau_{\gamma}\right.$ so that $\tau^{*}$ is canonical. Now $\left(q^{*}, \tau^{*}\right)$ is in $R$ because the 
statement $(*)$ above exactly proves that

$$
q^{*}(\gamma) \Vdash_{Q_{\gamma}} q^{*}(\gamma)\left\lceil\delta \Vdash _ { P _ { \delta } ^ { \gamma } } \tau ^ { * } \left\lceil\gamma \Vdash_{S(B)\lceil\gamma} \sup \left(q^{*}(\gamma)(\delta)\right) \in \rho_{\delta}^{\gamma} .\right.\right.
$$

We need a claim whose proof is a slight variation of the proof of Sublemma 7 , but it is similar enough that we omit it.

Sublemma 8. Let $\left\langle\left(q_{n}, \tau_{n}\right) \mid n<\omega\right\rangle$ be a decreasing sequence of elements of $Q_{\lambda+1} * S(B)$ where, for each $n<\omega, \tau_{2 n+1}$ is canonical and for each $\gamma<\lambda$ such that $P^{\lambda}$ is nontrivial

$$
\begin{aligned}
q_{2 n+1}\left\lceil\gamma \Vdash_{Q_{\gamma}} \forall \delta<\gamma^{++}\right. & \left(\text {if } q_{2 n}(\gamma)(\delta)\right. \text { is nontrivial } \\
& \text { then } q_{2 n+1}(\gamma)\left\lceil\delta \Vdash_{P_{\delta}^{\gamma}} \tau_{2 n+1}\left\lceil\gamma \Vdash_{S(B) \mid \gamma} \sup \left(q_{2 n}(\gamma)(\delta)\right) \in \rho_{\delta}^{\gamma}\right),\right.
\end{aligned}
$$

then there exists $\left(q^{*}, \tau^{*}\right)$ in $R$ below the sequence $\left\langle\left(q_{n}, \tau_{n}\right) \mid n<\omega\right\rangle$.

(The proof is like the proof of Sublemma 7. Define $q^{*}$ as there, and similarly for $\tau^{*}$.)

We now start the proof that $R$ is dense in $Q_{\lambda+1} * S(B)$. We need two technical lemmas.

Sublemma 9. $\chi<\gamma$ are cardinals such that $P^{\gamma}$ is nontrivial. (Hence $\gamma$ is a limit cardinal.) Suppose we are in $V^{Q_{\gamma}}$ given $p \in P^{\gamma}$, and $\tau \in S(B)\lceil\gamma$; then we can find (in $V^{Q_{\gamma}}$ ) a $p^{*} \leq p, \tau^{*} \leq \tau$, such that $\tau^{*}\left\lceil\chi=\tau\left\lceil\chi\right.\right.$ and for $\delta<\gamma^{++}$, if $p^{*}(\delta)$ is nontrivial, $p^{*}\left\lceil\delta \Vdash_{P_{\delta}^{\gamma}} \tau^{*} \Vdash_{S(B)\lceil\gamma} \sup \left(p^{*}(\delta)\right) \in \rho_{\delta}^{\gamma}\right.$.

Proof. $P_{\delta}^{\gamma}$ is obtained by iteration of length $\gamma^{++}$. We shall prove Sublemma 9 by proving by induction on $\eta \leq \gamma^{++}$that $P_{\eta}^{\gamma}$ satisfies the sublemma. (Of course now we just have the claim for $\delta<\eta$ rather than $\delta<\gamma^{++}$.) For successor $\eta$, say $\eta=\zeta+1$, we argue as follows. Consider $p(\zeta)$; if $p(\zeta)$ is trivial, then $p$ is essentially in $P_{\zeta}^{\gamma}$, and we can use the induction assumption for $\zeta$. If $p(\zeta)$ is nontrivial, then it is a $P_{\zeta}^{\gamma}$ term denoting a bounded closed set forced to be disjoint from $\tau_{\zeta}^{\gamma}$. Let $\dot{\mu}=\sup p(\zeta)$. ( $\dot{\mu}$ is actually a $P_{\zeta}^{\gamma}$ term.) In $V^{Q_{\gamma} * P_{\zeta}^{\gamma}}$, $S(B)\left\lceil\gamma\right.$ forces that $\rho_{\zeta}^{\gamma}$ is unbounded in $\gamma^{+}$. Hence we can find an extension of $\tau, \dot{\tau}^{\prime}$, which forces some member of $\rho_{\zeta}^{\gamma}, \dot{\mu}^{\prime}$, above $\dot{\mu}\left(\dot{\mu}^{\prime}\right.$ is also a term for an ordinal), but $\dot{\mu}^{\prime}$ is clearly forced to be outside of $\tau_{\zeta}^{\gamma}$. We claim that we can assume that $\stackrel{i}{\tau}^{\prime} \chi \chi=\tau\left\lceil\chi\right.$. Recall that the term $\rho_{\zeta}^{\gamma}$ was really an $S\left(C_{\gamma}\right)$ term, and let $\nu$ be a member of $C_{\gamma}$ above $\chi . S\left(C_{\gamma}\right)=S\left(C_{\gamma} \cap \nu+1\right) \times S\left(C_{\gamma}-\nu\right)$, but $S\left(C_{\gamma} \cap(\nu+1)\right)$ is of cardinality less than $\gamma$. Therefore in $S\left(C_{\gamma}\right)$ we can extend any condition, without changing its projection, to $S\left(C_{\gamma} \cap \nu+1\right)$ and force an ordinal in $\rho_{\zeta}^{\gamma}$ above $\mu$. If we consider the particular way in which we embedded $S\left(C_{\gamma}\right)$ into $S(B)\lceil\gamma$, this translates into: a condition in $S(B)\lceil\gamma$ can be extended to force a member of $\rho_{\zeta}^{\gamma}$ without changing its restriction to $\chi$.

Our next problem is that we want $\tau^{*}$ to be in $V^{Q_{\gamma}}$, not a $V^{P_{\zeta}^{\gamma}}$ term as $\dot{\tau}^{\prime}$ is. Since it is forced by $P_{\zeta}^{\gamma}$ that $S(B)\left\lceil\gamma \in V^{Q_{\gamma}}\right.$, we can find an extension of 
$p\left\lceil\zeta\right.$ such that, for some $\tilde{\tau} \in S(B)\left\lceil\gamma, p^{\prime} \Vdash \tilde{\tau}=\dot{\tau}^{\prime}\right.$. (Clearly $\tilde{\tau} \leq \dot{\tau}^{\prime}$.) We now apply the induction assumption to $\zeta, p^{\prime}$, and $\tau^{\prime}$ and get $p^{\prime \prime} \in P_{\zeta}^{\gamma}, p^{\prime \prime} \leq p^{\prime}$, $\tau^{\prime \prime} \leq \tilde{\tau}$ such that the sublemma holds for $\tau^{\prime \prime}, p^{\prime \prime}$. Define $\tau^{*}=\tau^{\prime \prime}, p^{*}\left\lceil\zeta=p^{\prime \prime}\right.$, and $p^{*}(\zeta)=$ a term denoting $p(\zeta) \cup\left\{\dot{\mu}^{\prime}\right\}$. It can be easily checked that all the requirements are satisfied.

For limit $\eta$, if $\operatorname{cf}(\eta)>\gamma$ then $p$ is actually in $P_{\zeta}^{\eta}$ for some $\zeta<\eta$, and we can use the induction assumption for $\zeta$. If $\operatorname{cf}(\eta) \leq \gamma$ then of course $\operatorname{cf}(\eta)<$ $\gamma$. Then since $P^{\gamma}$ is nontrivial, we can find some $\gamma>\chi^{\prime}>\chi+\operatorname{cf}(\eta)$. Let $\eta=\sup \left\langle\eta_{\alpha} \mid \alpha<\operatorname{cf}(\eta)\right\rangle$ where $\eta_{\alpha}$ are increasing and continuous. By induction on $\alpha \leq \operatorname{cf}(\eta)$ we define a decreasing sequence of conditions $p_{\alpha}$ in $P_{\eta}^{\gamma}$ and a decreasing sequence of elements of $S(B)\left\langle\tau_{\alpha} \mid \alpha \leq \operatorname{cf}(\eta)\right\rangle$ such that $\tau_{\alpha}\left\lceil\chi^{\prime}\right.$ is fixed, and $p_{\alpha+1}\left\lceil\eta_{\alpha}\right.$ and $\tau_{\alpha+1}$ satisfy the sublemma with respect to $P_{\eta_{\alpha}}^{\gamma^{\alpha}}$, where, for $\eta_{\alpha} \leq \delta, p_{\alpha}(\delta)=p_{0}(\delta)$. $p_{0}$ and $\tau_{0}$ are our given $p$ and $\tau$. For limit $\alpha, \tau_{\alpha}$ will be a lower bound of $\left\langle\tau_{\alpha} \mid \alpha<\operatorname{cf}(\eta)\right\rangle$, which exists since the $\tau_{\alpha}^{\prime}$ 's satisfy that the $\tau_{\alpha}\left\lceil\chi^{\prime}\right.$ are fixed and $\alpha<\chi^{\prime} . p_{\alpha}(\delta)$ for $\delta \geq \eta_{\alpha}$ is $p_{0}(\delta)$. (It still extends $p_{\beta}(\delta)$ for $\beta<\alpha$ since $p_{\beta}(\delta)$ was constantly equal to $p_{0}(\delta)$.) For $\delta<\eta_{\alpha}$ we let $p_{\alpha}(\delta)$ be a term denoting the union of $p_{\beta}(\delta), \beta<\alpha$, together with its sup. By induction on $\delta \leq \eta$ we should prove that $p_{\alpha}\left\lceil\delta\right.$ is in $P_{\eta}^{\gamma}$ where the only nontrivial point is to show as usual that, for $\delta<\eta_{\alpha}, \sup \left(\bigcup_{\beta<\alpha} p_{\beta}(\delta)\right)=\mu$ is forced to be outside of $\tau_{\delta}^{\gamma}$, but by our construction $\tau_{\alpha}$ extends $\tau_{\beta}$ for $\beta<\alpha$ and, for $\beta$ successor and $\delta<\eta_{\beta}, \tau_{\beta}$ forces $\sup \left(p_{\beta}(\delta)\right)$ to be in $\rho_{\delta}^{\gamma} \cdot \rho_{\delta}^{\gamma}$ is a term for a closed set; hence $\tau_{\alpha}$ forces $\mu$ to be in $\rho_{\delta}^{\gamma}$ (we use the fact that the $p_{\beta}(\delta)$ are forced by $p_{\alpha}\left\lceil\delta\right.$ to be increasing subsets of $\left.\gamma^{+}\right)$. As before we get that $\mu$ is forced by $p_{\alpha}\left\lceil\delta\right.$ to be out of $\tau_{\delta}^{\gamma}$. For $\alpha<\operatorname{cf}(\eta)$ we define $p_{\alpha+1}, \tau_{\alpha+1}$ to be an extension of $p_{\alpha}, \tau_{\alpha}$ such that $p_{\alpha+1}\left\lceil\eta_{\alpha}, \tau_{\alpha+1}\right.$ satisfy the requirements of the sublemma with respect to $\eta_{\alpha}, p_{\alpha}\left\lceil\eta_{\alpha}, \tau_{\alpha}\right.$, and, for $\delta \geq \eta_{\alpha}, p_{\alpha+1}(\delta)=p_{0}(\delta)$. (We use the induction assumption.) This concludes the inductive definition, and it is easily seen that $p_{\operatorname{cf}(\eta)}, \tau_{\operatorname{cf}(\eta)}$ witness the truth of the sublemma for $\eta$.

Sublemma 10. For $\gamma, \chi$ limit cardinals let $\chi<\gamma \leq \lambda$. Let $(q, \tau) \in Q_{\lambda+1} * S(B)$ such that $\tau$ is $\chi$ canonical. Then there exist $q^{*} \leq q$ and a $\gamma$ canonical term $\tau^{*}$ such that

(a) For $\gamma+1<\gamma^{\prime}$ or $\gamma^{\prime} \leq \chi, q^{*}\left(\gamma^{\prime}\right)=q\left(\gamma^{\prime}\right)$.

(b) $q^{*} \Vdash \tau^{*}\left\lceil\chi=\tau\left\lceil\chi \wedge \tau^{*} \leq \tau\right.\right.$ (hence $\left.\left(q^{*}, \tau^{*}\right) \leq(q, \tau)\right)$.

(c) For $\chi<\eta \leq \gamma+1$ if $\bar{P}^{\eta}$ is not trivial, then

$$
\begin{aligned}
q^{*}\left\lceil\gamma \Vdash_{Q_{\gamma}} \forall \delta<\eta^{++}\right. & \left(\text {if } q^{*}(\eta)(\delta)\right. \text { is not trivial } \\
& \text { then } q^{*}(\eta) \Vdash \delta \Vdash_{P_{\delta}^{\gamma}} \tau^{*}\left\lceil\eta \Vdash_{S(B)\lceil\eta} \sup \left(q^{*}(\eta)(\delta)\right) \in \rho_{\delta}^{\eta}\right) .
\end{aligned}
$$

(Note that we assume in (c) that $q^{*}\lceil\gamma$ forces the statement, not the natural $q^{*}\left\lceil\eta\right.$. The reason is that $\tau^{*}$ is not assumed to be canonical, so while we know that $\tau^{*}\left\lceil\eta \in V^{Q_{\eta}}\right.$ we do not have a $Q_{\eta}$ term forced to be equal to $\tau^{*}\lceil\eta$. Hence we need the information $q^{*}\lceil\gamma$.) 
Proof. The proof is by induction on $\gamma$ where the inductive assumption is that the statement of the sublemma holds for all $\chi<\gamma$. Sublemma 9 clearly takes care of the case where $\gamma$ is the minimal where $P^{\gamma}$ is nontrivial. (In this case $Q_{\gamma}$ is trivial, so the whole argument is taking place in $V$.) If $\gamma$ is a successor limit cardinal, say $\gamma=\eta^{+\omega}, \eta$ limit, then $P^{\gamma}$ is trivial, and the truth of the sublemma follows from the induction assumption for $\eta$. For $\gamma$ which is a limit of limit cardinals we distinguish two cases:

Case I: $\gamma$ is singular. Let $\gamma=\sup \left\langle\gamma_{\alpha} \mid \alpha<\operatorname{cf}(\gamma)\right\rangle$ (for simplicity we also put $\left.\gamma_{\operatorname{cf}(\gamma)}=\gamma\right)$, where the $\gamma_{\alpha}$ 's are increasing and continuous where $\chi<\gamma_{0}$, $\operatorname{cf}(\gamma)<\gamma_{0}$, and each $\gamma_{\alpha}$ is a limit cardinal. Let $\left(q_{0}, \tau_{0}\right) \leq(q, \tau)$ satisfy the sublemma with respect to $\chi$, and $\gamma_{0}$. We are going to define by induction on $\alpha \leq \operatorname{cf}(\gamma)$ a decreasing sequence $\left(q_{\alpha}, \tau_{\alpha}\right)$ such that $\tau_{\alpha}$ is $\gamma_{\alpha}$ canonical, and $q_{\alpha}(\delta)$ is modified only once (when $\left.\gamma_{\alpha} \leq \delta<\gamma_{\alpha+1}\right)$. We shall have $\tau_{\alpha}\left\lceil\gamma_{0}=\right.$ $\tau_{0}\left\lceil\gamma_{0}\right.$. For successor $\alpha=\beta+1,\left(q_{\alpha}, \tau_{\alpha}\right)$ is defined as an extension of $\left(q_{\beta}, \tau_{\beta}\right)$ satisfying the sublemma for $\gamma_{\beta+1}$ for $\gamma, \gamma_{\beta}$ for $\chi$. (Note that the only changes in $q_{\beta}(\delta)$ when we pass to $q_{\beta+1}(\delta)$ are for $\gamma_{\beta}<\delta \leq \gamma_{\beta+1}$.) For limit $\alpha$, let $\tau_{\alpha}^{*}$ be the term which is forced to be the minimal lower bound of $\tau_{\beta}, \beta<\alpha$. Note that it satisfies $\tau_{\alpha}^{*}\left\lceil\gamma_{0}=\tau_{0}\left\lceil\gamma_{0}\right.\right.$. It exists since $\alpha \leq \operatorname{cf}(\gamma)<\gamma_{0} . \quad q_{\alpha}^{*}(\delta)$ will be the limiting value of $q_{\beta}(\delta), \beta<\alpha\left(q_{\beta}(\delta)\right.$ actually obtains only two values, and one of them is eventually obtained). Also note that since each $\tau_{\beta}$ for $\beta<\alpha$ was $\gamma_{\beta}$ canonical, it was $\gamma_{\alpha}$ canonical. We get that $\tau_{\alpha}^{*}$ is $\gamma_{\alpha}$ canonical, and $q_{\alpha}^{*}(\delta)$ and $\tau_{\alpha}^{*}$ are almost what we need for $\eta<\gamma_{\alpha}$. We still have to handle $\gamma_{\alpha}$ itself. So if $P^{\gamma_{\alpha}}$ is trivial we put $q_{\alpha}=q_{\alpha}^{*}, \tau_{\alpha}=\tau_{\alpha}^{*}$. If $P^{\gamma_{\alpha}}$ is nontrivial, we consider $p=q_{\alpha}^{*}\left(\eta_{\alpha}\right)=q_{0}\left(\eta_{\alpha}\right)$ as an element of $V^{Q_{n_{\alpha}}}$, where we assume that $q^{*}\left\lceil\eta_{\alpha} \in G\left(Q_{\eta_{\alpha}}\right)\right.$ and apply Sublemma 9 to $p$ and $\tau_{\alpha}^{*}\lceil\gamma$ (replace $\chi$ by $\gamma_{0}$ ). Note that $\tau_{\alpha}^{*}$ is a $Q_{\gamma_{\alpha}}$ term, so Lemma 9 is applicable. We get $p^{*}$ and $\tau^{* *}$ which we can consider now (coming back to $V$ ) as $Q_{\gamma_{\alpha}}$ terms forced to denote a pair satisfying the requirements of Sublemma 9. Define $q_{\alpha}$ by $q_{\alpha}\left\lceil\gamma_{\alpha}=q^{*}\left\lceil\gamma_{\alpha}, q_{\alpha}\left(\gamma_{\alpha}\right)=p^{*}, q_{\alpha}(\delta)=q_{\alpha}^{*}(\delta)=q_{0}(\boldsymbol{\delta})\right.\right.$ for $\delta>\gamma_{\alpha} . \tau_{\alpha}$ is the term denoting the member of $S(B)$ whose restriction to $\gamma_{\alpha}$ is $\tau^{* *}$, and above $\gamma_{\alpha}$ it is like $\tau_{\alpha}^{*}$. It is obvious that $\tau_{\alpha}$ is $\gamma_{\alpha}$ canonical. We can easily show that, for $\alpha \leq \operatorname{cf}(\gamma),\left(q_{\alpha}, \tau_{\alpha}\right)$ satisfy the sublemma for $\gamma_{\alpha}$. In particular $\left(q_{\mathrm{cf}(\gamma)}, \tau_{\mathrm{cf}(\gamma)}\right)$, is the required pair for $\gamma$.

Case II: $\gamma$ is regular. In this case we know that the support of $q$ is nonstationary in $\gamma$, so let $\left\langle\gamma_{\alpha} \mid \alpha \leq \gamma\right\rangle$ be a continuous increasing sequence of limit cardinals, where $\gamma_{\gamma}=\gamma, \chi<\gamma_{0}$, and $q\left(\gamma_{\alpha}\right)$ is the trivial condition of $P^{\gamma_{\alpha}}$. We define by induction for $\alpha \leq \gamma$ a decreasing sequence $\left\langle q_{\alpha}, \tau_{\alpha}\right\rangle$ all below $\langle q, \tau\rangle$ such that $\left\langle q_{\alpha}, \tau_{\alpha}\right\rangle$ satisfies the sublemma with respect to $\gamma_{\alpha}$, and if $\alpha<\beta$ then $\tau_{\beta}\left\lceil\gamma_{\alpha}=\tau_{\alpha}\left\lceil\gamma_{\alpha} .\left\langle q_{0}, \tau_{0}\right\rangle\right.\right.$ is any extension of $\langle q, \tau\rangle$ satisfying the sublemma with respect to $\chi$ and $\gamma_{0}$. For successor $\alpha=\beta+1,\left\langle q_{\beta+1}, \tau_{\beta+1}\right\rangle$ is an extension of $\left\langle q_{\beta}, \tau_{\beta}\right\rangle$ satisfying the lemma for $\gamma_{\beta}$ (instead of $\chi$ ) and $\gamma_{\beta+1}$. For limit $\alpha$ we let $\tau_{\alpha}$ be the maximal lower bound of $\left\langle\tau_{\beta} \mid \beta<\alpha\right\rangle$. It exists since $\tau_{\beta}\left\lceil\gamma_{\mu}\right.$ is eventually constant for $\mu<\alpha$. (Note that $\operatorname{Col}\left(\gamma_{\alpha},<\zeta\right.$ ) 
never appears as a component in $S(B)$ even if $\gamma_{\alpha}$ is regular; hence for any $\operatorname{Col}(\mu,<\zeta)$ appearing in $S(B)$ we have $\mu<\gamma_{\alpha}$ or $\gamma_{\alpha}<\mu$, in which case we have enough completeness because $\alpha \leq \gamma_{\alpha}$.) $q_{\alpha}(\delta)$ is defined to be $q_{\beta}(\delta)$ for $\beta$ large enough below $\alpha$. $\tau_{\alpha}$ can be easily picked to be $\gamma_{\alpha}$ canonical, using the fact that for $\beta<\alpha, \tau_{\beta}$ is $\gamma_{\beta}$ canonical. (Note that $q_{\beta}(\delta)$ is changed only once when $\gamma_{\beta} \leq \delta<\gamma_{\beta+1}$.) Note that $q_{\alpha}\left(\gamma_{\alpha}\right)$ is still the trivial condition (we never changed it), and hence $\left(q_{\alpha}, \tau_{\alpha}\right)$ clearly satisfies the sublemma for $\chi$ and $\gamma_{\alpha}$. Considering $\left(q_{\gamma}, \tau_{\gamma}\right)$ establishes this inductive step.

Sublemma 11. $R$ is dense in $Q_{\lambda+1} * S(B)$.

Proof. By induction define a decreasing sequence of conditions in $Q_{\lambda+1} * S(B)$. $\left\langle\left(q_{n}, \tau_{n}\right) \mid n<\omega\right\rangle$. Suppose we are given $(q, \tau)$, where by Lemma 6 we can assume that $\tau$ is canonical. Let $\left(q_{0}, \tau_{0}\right)$ satisfy Sublemma 10 where $\gamma=\lambda$, $\chi=0$ for $(q, \tau)$. In general, given $\left(q_{2 n}, \tau_{2 n}\right)$, let $\left(q_{2 n+1}, \tau_{2 n+1}\right)$ be such that $q_{2 n+1} \leq q_{2 n}, \tau_{2 n+1}$ is canonical, and $q_{2 n+1} \Vdash \tau_{2 n}=\tau_{2 n+1}$. (Hence we have $\left.\left(q_{2 n+1}, \tau_{2 n+1}\right) \leq\left(q_{2 n}, \tau_{2 n}\right).\right) \quad\left(q_{2 n+2}, \tau_{2 n+2}\right)$ will be an extension of $\left(q_{2 n+1}, \tau_{2 n+1}\right)$ satisfying Sublemma 10 with $\gamma=\lambda, \chi=0$ with respect to $\left(q_{2 n+1}, \tau_{2 n+1}\right)$. We know that for $\eta \leq \lambda$, for $\eta \leq \gamma+1$ for which $P^{\eta}$ is nontrivial

$$
\begin{aligned}
q_{2 n} \Vdash_{Q_{\lambda+1}} \forall \delta<\eta^{++} & \text {(if } q_{2 n}(\eta)(\delta) \text { is not trivial, then } \\
& q_{2 n}(\eta)\left\lceil\delta \Vdash \tau_{2 n}^{*}\left\lceil\eta \Vdash_{S(B) \mid \eta} \sup \left(q_{2 n}(\eta)(\delta)\right) \in \rho_{\delta}^{\eta}\right),\right.
\end{aligned}
$$

but $q_{2 n+1} \Vdash \tau_{2 n}=\tau_{2 n+1}$ and $\tau_{2 n+1}$ is canonical. Hence $\tau_{2 n+1}\left\lceil\eta\right.$ is a $Q_{\eta}$ term, and once we know that $\tau_{2 n+1}\lceil\eta$, the truth of $(* *)$ just depends on the value of $G\left(Q_{\eta}\right)$. Hence

$$
\begin{aligned}
& q_{2 n+1}\left\lceil\eta \Vdash \forall \delta<\eta^{++}\left(\text {if } q_{2 n}(\eta)(\delta)\right. \text { is nontrivial, then }\right. \\
& \qquad q_{2 n}\left\lceil(\eta)(\delta) \Vdash_{P_{\delta}^{\eta}} \tau_{2 n+1}\left\lceil\eta \Vdash_{S(B)\lceil\eta} \sup \left(q_{2 n}(\eta)(\delta)\right) \in \rho_{\delta}^{\gamma}\right),\right.
\end{aligned}
$$

which exactly proves that the conditions of Sublemma 8 are satisfied. Hence we get that there is $\left(q^{*}, \tau^{*}\right) \in R$ below $\left\langle\left(q_{n}, \tau_{n}\right) \mid n<\omega\right\rangle$; in particular, we get an element of $R$ below $(q, \tau)$.

This completes at last the proof of Lemma 5.

Recall from $\S 3$ that $S_{n}$ is the product $\times_{n \leq l<\omega} \operatorname{Col}\left(\kappa_{l}^{+2},<\kappa_{l+1}\right) . S_{n}$ is of course $\kappa_{n}$ directed closed; hence by Lemma 5 if $B$ is adequate and $\sup (B)=\lambda$, then $Q_{\lambda+1} * S(B) * S_{n}$ is $\kappa_{n}$ directed closed. (Of course the last iteration is really a product because $S_{n}$ of $V^{Q_{\lambda+1}^{* B}}$ is the same as $S_{n}$ in the sense of $V$.) $S(B)$ already belongs to $V^{Q_{\lambda+1}}$. Note that $Q_{\lambda+2}^{\infty}$ is $\lambda^{+\omega+1}$ distributive. (It does not introduce any new sequences of ordinals of length $\leq \lambda^{+\omega}$.) Since the cardinality of $S(B) * S_{n}$ is less than $\lambda^{+\omega}, P^{m}\left(\lambda^{+k}\right)$ in the sense of $V^{Q_{\lambda+1} * S(B) * S_{n}}$ is the same as in $V^{Q * B * S_{n}}$ (for $m, k<\omega$ ), but $Q_{\lambda+1} * B * S_{n}$ is essentially $\kappa_{n}$ directed closed. Hence by our assumptions about $\kappa_{n}$

$$
V^{Q_{\lambda+1} * S(B) * S_{n}} \vDash \kappa_{n} \text { is supercompact }
$$


In particular,

$$
V^{Q_{\lambda+1} * S(B) * S_{n}} \vDash \kappa_{n} \text { is } \lambda^{+5} \text { supercompact . }
$$

But the fact that $\kappa_{n}$ is $\lambda^{+5}$ supercompact is witnessed by a subset of $P\left(P\left(\lambda^{+5}\right)\right)$, namely the normal ultrafilter on $P_{\kappa_{n}}\left(\lambda^{+5}\right)$, but this ultrafilter is also a normal ultrafilter in $V^{Q * S(B) * S_{n}}$. Hence we have proved

Lemma 12. If $Q$ was the forcing notion for killing all the bad stationary sets, then in $V^{Q}$ the following facts hold:

(1) G.C.H. holds above $\kappa_{\omega}$, and $2^{\kappa_{n}}=\kappa_{n}^{+}$for $n<\omega$.

(2) There are no bad stationary sets.

(3) If $B$ is 1-adequate with $\sup (B)=\lambda$ a limit cardinal, then if we force with $S(B) * S_{n}, \kappa_{n}$ is $\lambda^{+5}$ supercompact.

We shall never have to refer again to the particular way in which we defined $Q$; the only facts we shall need about $V^{Q}$ are summarized by (1), (2), (3) in the statement of Lemma 12. Therefore from now on we assume that our ground model $V$ satisfies (1)-(3) of Lemma 12.

Definition 4. Given an adequate set of cardinals $B$ with $\sup (B)=\lambda$, a sequence of normal ultrafilters $\left\langle U_{n} \mid n<\omega\right\rangle$ such that $U_{n}$ is a normal ultrafilter on $\kappa_{n}$ and a sequence of functions $\left\langle f_{n} \mid n<\omega\right\rangle \quad\left(f_{n}: \kappa_{n} \rightarrow \kappa_{n}\right)$ are called good for $B$ if for $n<\omega$ every condition in $S(B) * S_{n}$ forces that " $U_{n}$ is the projection of some normal ultrafilter on $P_{\kappa_{n}}\left(\lambda^{+}\right), F_{n}$, such that

$$
\left\{P \mid \text { the order type of } P=f_{n}\left(P \cap \kappa_{n}\right)\right\} \in F_{n} \text { ". }
$$

Lemma 13. There exist a sequence of normal ultrafilters $\left\langle U_{n} \mid n<\omega\right\rangle$ and a sequence of functions $\left\langle f_{n} \mid n<\omega\right\rangle$ which are good for unboundedly many sets of cardinality $\leq \kappa_{\omega}^{+}$; namely for every set of cardinals $B,|B| \leq \kappa_{\omega}^{+}$(each member of $B$ above $\left.\kappa_{\omega}\right)$, there exists an adequate $B^{*}$ with $\sup \lambda$ which is $a$ limit cardinal, $B \subseteq B^{*}$ such that $\left\langle U_{n} \mid n<\omega\right\rangle,\left\langle f_{n} \mid n<\omega\right\rangle$ are good for $B^{*}$.

Proof. Assume the lemma fails; then for every sequence of ultrafilters $\vec{U}=$ $\left\langle U_{n} \mid n<\omega\right\rangle \quad\left(U_{n}\right.$ on $\left.\kappa_{n}\right)$ and a sequence of functions $\vec{f}=\left\langle f_{n} \mid n<\omega\right\rangle \quad\left(f_{n}\right.$ : $\left.\kappa_{n} \rightarrow \kappa_{n}\right)$, there is $B(\vec{U}, \vec{f})$ of cardinality $\leq \kappa_{\omega}^{+}$such that for no adequate $B^{*}, B \subseteq B^{*}, \vec{U}, \vec{f}$ are good for $B^{*}$. Let $T$ be the union of all the $B(\vec{U}, \vec{f})$ since there are at most $\kappa_{\omega}^{+}$sequences of the form $\vec{U}$ and $\vec{f}$ we consider, and since $|B(\vec{U}, \vec{f})| \leq \kappa_{\omega}^{+}$, the cardinality of $T$ is at most $\kappa_{\omega}^{+}$, but then we can find an adequate $B^{*}, T \subseteq B^{*}$, with $\sup \left(B^{*}\right)$ a limit cardinal $\lambda$.

In $V^{S\left(B^{*}\right) * S_{n}}, \kappa_{n}$ is $\lambda^{+5}$ supercompact. By a theorem of Solovay (see [S-R$\mathrm{K}, \mathrm{Men}$ ]; see also [La] for similar arguments) there is a normal ultrafilter on $P_{\kappa_{n}}\left(\lambda^{+}\right), F_{n}$, and a function $f_{n}: \kappa_{n} \rightarrow \kappa_{n}$ such that

$$
\left\{P \mid \text { the order type of } P=f_{n}\left(P \cap \kappa_{n}\right)\right\} \in F_{n} \text {. }
$$


Let $U_{n}$ be the projection of $F_{n}$ to $\kappa_{n}$. But $S(B) * S_{n}$ is $\kappa_{n}^{+2}$ closed and $2^{\kappa_{n}}=\kappa_{n}^{+}$; hence both $U_{n}$ and $f_{n}$ are in $V$. Therefore some condition in $S(B) * S_{n}$ forces

(*) " $U_{n}$ can be extended to a normal ultrafilter $F_{n}$ on $f_{\kappa_{n}}\left(\lambda^{+}\right)$and $\{P \mid$ order type of $\left.P=f_{n}\left(P \cap \kappa_{n}\right)\right\} \in F_{n}$ ".

By the fact that $S(B) * S_{n}^{n}$ is a homogeneous forcing notion, every condition in $S(B) * S_{n}$ forces $(*)$ above. (Note that we can get the sequences $\left\langle U_{n} \mid n<\omega\right\rangle$ and $\left\langle f_{n}\right| n\langle\omega\rangle$ in $V$.) We showed that $\vec{U}=\left\langle U_{n}\right| n\langle\omega\rangle$ and $\vec{f}=\left\langle f_{n} \mid n<\omega\right\rangle$ are good for $B^{*}$, but $B(\vec{U}, \vec{f}) \subseteq B^{*}$, a contradiction to the definition of $B(\vec{U}, \vec{f})$.

We are ready for the proof of Theorem 1. Fix the sequence $\left\langle U_{n} \mid n<\omega\right\rangle$, $\left\langle f_{n} \mid n<\omega\right\rangle$ satisfying Lemma 13 . We use the forcing notion $\mathscr{P}$ described in $\S 3$ in terms of the sequences $\left\langle U_{n} \mid n<\omega\right\rangle$ and $\left\langle f_{n} \mid n<\omega\right\rangle$ (see the remarks after the proof of Theorem 3.1).

So let $\mathscr{P}$ be as above (defined in terms of $\vec{U}, \vec{f}$ ). We claim that in $V^{\mathscr{P}}$ we have that $\kappa_{\omega}$ is the first cardinal fixed point that G.C.H. holds and that $\Delta_{\kappa_{\omega, \mu}}^{-}$ holds for all $\kappa_{\omega}<\mu$ ( $\mu$ regular).

Lemma 14. For all $n<\omega$,

$$
\begin{aligned}
H_{n} & =\left\{\alpha \mid \alpha<\kappa_{n}, \text { G. C. H. holds between (inclusive) } \alpha \text { and } f_{n}(\alpha)\right\} \\
& \in U_{n}
\end{aligned}
$$

and

$$
\begin{aligned}
L_{n} & =\left\{\alpha \mid \alpha<\kappa_{n}, \text { there are exactly } \alpha^{+\omega+1} \text { cardinals between } \alpha \text { and } f_{n}(\alpha)\right\} \\
& \in U_{n} .
\end{aligned}
$$

Proof. Pick any adequate $B$ such that $\vec{U}, \vec{f}$ is $\operatorname{good}$ for $B(\sup (B)=\lambda)$. In $V^{S(B) * S_{n}}, \kappa_{n}$ is $\lambda^{+}$supercompact, and there is a normal ultrafilter on $P_{\kappa_{n}}\left(\lambda^{+}\right), F_{n}$, such that $F_{n}$ projects to $U_{n}$ and $\left\{P \mid P \in P_{\kappa_{n}}\left(\lambda^{+}\right)\right.$, order type of $\left.P=f_{n}\left(P \cap \kappa_{n}\right)\right\} \in F_{n}$. In $V^{S(B) * S_{n}}$ form the ultrapower of the universe by $F_{n}$ and get a transitive class $M$. The ordinal $\lambda^{+}$is represented in the ultrapower by the function $g(P)=$ order type of $P\left(P \in P_{\kappa_{n}}\left(\lambda^{+}\right)\right)$; hence by our assumption we can take $g$ to be $f_{n}\left(P \cap \kappa_{n}\right) . \kappa_{n}$ is represented by the function $h(P)=P \cap \kappa_{n}$ (see [S-R-K]). Also $P(\lambda)$ (in the sense of $V^{S(B) * S_{n}}$ ) is in $M$. In $V^{S(B) * S_{n}}$ G.C.H. holds between $\kappa_{n}$ and $\lambda$. (Note that above $\kappa_{\omega}$ G. C. H. holds in $V ; S(B) * S_{n}$ does not destroy it.) Between $\kappa_{n}$ and $\kappa_{\omega}$ we forced with $\times_{n \leq i} \operatorname{Col}\left(\kappa_{i}^{+2},<\kappa_{i+1}\right)$, each $\kappa_{i}$ satisfies the G.C.H., and when we force with $\operatorname{Col}\left(\kappa_{i}^{+2},<\kappa_{i+1}\right)$ all cardinals between $\kappa_{k}^{+1}$ and $<\kappa_{i+1}$ satisfy the G.C.H. Therefore

$$
V^{S(B) * S_{n}} \vDash \forall \alpha\left[\left(\kappa_{n} \leq \alpha \leq \lambda\right) 2^{\alpha}=\alpha^{+}\right] .
$$

Since, for $\alpha<\lambda, P(\alpha) \in M$,

$$
M \vDash \forall \alpha\left(\kappa_{n} \leq \alpha \leq \lambda\right) 2^{\alpha}=\alpha^{+} .
$$


By the Los theorem applied to our ultrapower

$$
\left\{P \mid \forall \beta P \cap \kappa_{n} \leq \beta<f\left(P \cap \kappa_{n}\right), 2^{\beta}=\beta^{+}\right\} \in F_{n} .
$$

By definition of the projection of $F_{n}$, we get (in $V^{S(B) * S_{n}}$ )

$$
\left\{\alpha \mid \alpha<\kappa_{n}, \forall \beta \alpha \leq \beta<f(\alpha), 2^{\beta}=\beta^{+}\right\} \in U_{n},
$$

but $U_{n} \in V$ and, for $\beta<\kappa_{n}, 2^{\beta}=\beta^{+}$in $V^{S(B) * S_{n}}$ if and only if it is true in $V$. (Forcing with $S(B) * S_{n}$ does not introduce any new subset of $\kappa_{n}$.) Hence we proved

$$
\left\{\alpha \mid \alpha<\kappa_{n} \text {, G. C. H. holds between } \alpha \text { and } f_{n}(\alpha) \text { inclusive }\right\} \in U_{n} .
$$

For the second fact we again pick an adequate $B$ such that $\left\langle U_{n} \mid n<\omega\right\rangle$ and $\left\langle f_{n}\right| n\langle\omega\rangle$ are good for $B$; hence in $V^{S(B)} \kappa_{n}$ is $\lambda^{+}$supercompact by a normal ultrafilter $F_{n}$ projecting to $U_{n}$ and satisfying

$$
\left\{P \mid P \in P_{\kappa_{n}}\left(\lambda^{+}\right), \text {order type of } P=f_{n}\left(P \cap \kappa_{n}\right)\right\} \in F_{n} .
$$

Again if $M$ is the ultrapower of $V^{S(B) * S_{n}}$ by $F_{n}$, then $P(\lambda) \in M$, and hence, for $\alpha \leq \lambda, \alpha$ is a cardinal in $M$ iff it is a cardinal in $V^{S(B) * S_{n}}$. But in $V^{S(B) * S_{n}}$ $\kappa_{\omega}=\kappa_{n}^{+\omega}, \kappa_{\omega}^{+}=\kappa_{n}^{+\omega+1}$ and for $\kappa_{\omega}<\mu$ if $\mu \leq \lambda$ is a cardinal in $V^{S(B) * S_{n}}$, then it is either in the closure of $B$ or successor of an element in the closure of $B . \lambda^{+}$is represented in $M$ by the function $g(P)=f_{n}\left(P \cap \kappa_{n}\right)$. Also every limit point of $B$ is still a cardinal in $V^{S(B) * S_{n}}$; hence if $B$ is of cardinality $\kappa_{\omega}^{+}$ (which we can assume without loss of generality), then

$$
V^{S(B) * S_{n}} \vDash \text { there are exactly } \kappa_{\omega}^{+}=\kappa_{n}^{+\omega+1} \text { many cardinals between } \kappa_{n}^{+} \text {and } \lambda \text {. }
$$

Therefore

$$
M \vDash \text { there are exactly } \kappa_{n}^{+\omega+1} \text { many cardinals between } \kappa_{n} \text { and } \lambda .
$$

Using the Los theorem

$$
\begin{aligned}
& \left\{P \mid P \in P_{\kappa_{n}}\left(\lambda^{+}\right), \text {there are exactly }\left(P \cap \kappa_{n}\right)^{+\omega+1}\right. \\
& \left.\quad \text { many cardinals between } P \cap \kappa_{n} \text { and } f\left(P \cap \kappa_{n}\right)\right\} \in F_{n} .
\end{aligned}
$$

$F_{n}$ projects to $U_{n}$ so we get

$L_{n}=\left\{\alpha \mid \alpha<\kappa_{n}\right.$, there are exactly $\alpha^{+\omega+1}$ many cardinals between $\alpha$ and $\left.f_{n}(\alpha)\right\}$

$\in U_{n}$.

Let $p$ be a condition in $P$ whose $\alpha$-part, $f$-part, and $S$-part are trivial and whose $A$-part is $\left\langle L_{n} \cap H_{n} \mid n<\omega\right\rangle$ (where $L_{n}$ and $H_{n}$ were defined by the statements of Lemma 14).

Lemma 15. In $V^{\mathscr{P}}$ (if $p \in G(\mathscr{P})$ ) $\kappa_{\omega}$ is the first cardinal fixed point and G. C. H. holds.

Proof. By the analysis done in $\S 3$ we know that in $V^{\mathscr{P}}$ each $\kappa_{n}$ is a successor cardinal, and the cardinals below $\kappa_{0}$ are exactly $\omega, \omega_{1}, \alpha_{0}$, all cardinals in $V$ 
between $\alpha_{0}$ and $f_{0}\left(\alpha_{0}\right), f_{0}\left(\alpha_{0}\right), f\left(\alpha_{0}\right)^{+}$, and $\kappa_{0}$. The cardinals between $\kappa_{n}$ and $\kappa_{n+1}$ are $\kappa_{n}, \kappa_{n}^{+}, \kappa_{n}^{++}, \alpha_{n+1}$, all cardinals $V$ between $\alpha_{n+1}$ and $f_{n+1}\left(\alpha_{n+1}\right)$, $f_{n+1}\left(\alpha_{n+1}\right), f_{n+1}\left(\alpha_{n+1}\right)^{+}$, and $\kappa_{n+1}$ (where $\left\langle\alpha_{n} \mid n<\omega\right\rangle$ is the sequence introduced by the $\alpha$-parts of conditions in $G(\mathscr{P}))$. Since $p \in G(\mathscr{P})$, we know $\alpha_{i} \in L_{i} \cap H_{i}$, so there are exactly $\alpha_{i}^{+\omega+1}$ cardinals (in $V$ ) between $\alpha_{i}$ and $f_{i}\left(\alpha_{i}\right)$, and so none of them is a cardinal fixed point. It is now obvious that there are no cardinal fixed points below $\kappa_{\omega}$. On the other hand, there are at least $\sup \left(\left\{\alpha_{i}^{+\omega+1} \mid i<\omega\right\}\right)$ many cardinals below $\kappa_{\omega}$, but the last sup is $\kappa_{\omega}$, so $\kappa_{\omega}$ is a cardinal fixed point.

Similarly the fact that G.C.H. was satisfied in $V$ by $\kappa_{\omega}$ and between $\alpha_{n}$ and $f_{n}\left(\alpha_{n}\right)$ (recall $\alpha_{n} \in L_{n} \cap H_{n}$ ) guarantees the G.C.H. below $\kappa_{\omega}$. Above $\kappa_{\omega}$ it holds because it holds in $V$. (Between $\omega_{1}$ and $\alpha_{0}$ we forced with $\operatorname{Col}\left(\omega_{1},<\alpha_{0}\right)$ which arranged G.C.H. below $\alpha_{0}$; between $\left(f \alpha_{0}\right)^{+}$and $\kappa_{0}$ we used $\operatorname{Col}\left(f\left(\alpha_{0}\right)^{+}, \kappa_{0}\right)$ which arranged G.C.H. between $f\left(\alpha_{0}\right)$ and $\kappa_{0}$, etc.).

Lemma 16. $V^{\mathscr{P}} \vDash \Delta_{\kappa_{\omega}, \mu}$ holds for all regular $\mu>\kappa_{\omega}$ such that $\mu$ is not a successor of a singular cardinal of cofinality bigger than $\kappa_{\omega}^{+}$and it is not nonMahlo inaccessible.

Proof. Let $\mu$ be as in the statement of the lemma, and let $\dot{\tau}, \dot{S}$ be $\mathscr{P}$ terms, $q \in \mathscr{P}$, where $q \Vdash \dot{\tau}$ is an algebra on $\mu$ with $<\kappa_{\omega}$ many operations and $\dot{S}$ is a stationary subset of $\mu$ such that if $\alpha \in \dot{S}$ then $\operatorname{cf}(\alpha)<\kappa_{\omega}$.

The forcing notion $\mathscr{P}$ of course is a forcing notion even in $V^{S(B)}$ where $B$ is any adequate set. (In fact $\left\langle U_{n} \mid n<\omega\right\rangle$ is still a sequence of normal ultrafilters in $V^{S(B)}$.) And therefore the terms $\dot{\tau}$ and $\dot{S}$ are $\mathscr{P}$ terms also in $V^{S(B)}$. Clearly we still have

$$
q \Vdash \tau \text { is an algebra on } \mu \text { with }<\kappa_{\omega} \text { many operations }
$$

when the forcing is over $V^{S(B)}$. We claim that we can pick $B$ such that in $V^{S(B)}$ we have

$$
q^{*} \Vdash S \text { is a stationary subset of } \mu \text { and } \mu \text { is regular }
$$

for appropriate choice of $q^{*} \leq q$ and such that $\left\langle U_{n} \mid n<\omega\right\rangle$ and $\left\langle f_{n} \mid n<\omega\right\rangle$ are good for $B$. It is clear in case $\mu=\kappa_{\omega}^{+}$for any $B$ because in $V^{S(B)}$ we do not have a new subset of $\kappa_{\omega}^{+}$. (Recall that, for an adequate $B, \min (B) \geq \kappa_{\omega}^{++}$.) Hence, since $|\mathscr{P}|=\kappa_{\omega}^{+}$, there are no new $\mathscr{P}$ terms for subsets of $\kappa_{\omega}^{+}$, so if there is a term in $V^{S(B)}$ for a closed unbounded subset of $\kappa_{\omega}^{+}$disjoint from $\dot{S}$, this term is in $V$, and it is a term for a closed unbounded subset disjoint from $\dot{S}$, which is a contradiction.

For $\mu>\kappa_{\omega}^{+}$, there is $q^{*}$ such that (over $V$ ) $A=\left\{\alpha \mid q^{*} \Vdash \alpha \in \dot{S}\right\}$ is a stationary subset of $A$. This is obvious since $|\mathscr{P}|=\kappa_{\omega}^{+}$and $\mu>\kappa_{\omega}^{+} . A$ is a stationary subset of $\mu$ in $V$; hence by our assumptions it is not bad. Hence we can find adequate $C$ such that $V^{S(C)} \vDash A$ is a stationary subset of $\mu$. Without loss of generality we can assume that $V^{S(C)} \vDash \mu$ is regular. The reason for it 
is that if $\mu$ is not non-Mahlo inaccessible and not a successor of a singular cardinal we can assume that $\mu \in C$. (Note that if an adequate $C$ works, any bigger $C$ works.) If $\mu=\delta^{+}$, where $\operatorname{cf}(\delta) \leq \kappa_{\omega}^{+}$, then we can have that $C$ is cofinal in $\delta$; hence $S(C)$ does not collapse $\mu$. By definition of $\left\langle U_{n} \mid n<\omega\right\rangle$, $\left\langle f_{n} \mid n<\omega\right\rangle$ we can find adequate $B, C \subseteq B$, such that $\left\langle U_{n} \mid n<\omega\right\rangle,\left\langle f_{n} \mid n<\omega\right\rangle$ are good for $B$. Hence a generic filter for $S(C)$ picks a generic filter for $S(B)$. Therefore we must have $V^{S(B)} \vDash A$ is stationary. (A club disjoint from $A$ in $V^{S(B)}$ is in $V^{S(C)}$.) Since $|\mathscr{P}|=\kappa_{\omega}^{+}<\mu$, we have $V^{S(B) * \mathscr{P}} \vDash A$ is stationary. But $q^{*} \Vdash A \subseteq \dot{S}$ (also over $V^{S(B)}$ ); hence over $S(B)$ we get $q^{*} \Vdash \dot{S}$ is a stationary subset of $\mu$ and that $\mu$ is regular and hence $\mu$ is regular in $V^{S(B) * S_{n}}$ for every $n$.

Fix $n<\omega$. We know that in $V^{S(B) * S_{n}}$ there is a normal ultrafilter on $P_{\kappa_{n}}\left(\lambda^{+}\right), F_{n}$, such that $F_{n}$ projects to $U_{n}$ and $\left\{P \mid P \in P_{\kappa_{n}}\left(\lambda^{+}\right)\right.$, order type of $\left.P=f_{n}\left(P \cap \kappa_{n}\right)\right\} \in F_{n}$. If $F_{n}^{*}$ is the projection of $F_{n}$ to $P_{\kappa_{n}}(\mu)$ we clearly have the following: In $V^{S(B) * S_{n}} \vDash$ there is a normal ultrafilter on $P_{\kappa_{n}}(\mu), F_{n}^{*}$, whose projection to $\kappa_{n}$ is $U_{n}$ and such that $\left\{P \mid P \in P_{\kappa_{n}}(\mu)\right.$, order type of $\left.P \leq f_{n}\left(P \cap \kappa_{n}\right)\right\} \in F_{n}^{*}$. We have shown that in $V^{S(B)}$ the sequences $\left\langle\kappa_{n} \mid n<\omega\right\rangle$, $\left\langle U_{n} \mid n<\omega\right\rangle,\left\langle f_{n} \mid n<\omega\right\rangle$, and the cardinal $\mu$ satisfy all the assumptions of Theorem 3.15, where the forcing notion mentioned there, $\mathscr{P}$, is exactly our given $\mathscr{P} .\left(\mathscr{P}\right.$ defined from $\left\langle U_{n} \mid n<\omega\right\rangle$ and $\left\langle f_{n} \mid n<\omega\right\rangle$ is the same in $V$ and $V^{S(B)}$, since forcing with $S(B)$ does not introduce any new sequences of length $\leq \kappa_{\omega}^{+}$.) Therefore by Theorem 3.15, applied in $V^{S(B)}$, we know that $V^{S(B) * \mathscr{P}} \vDash \Delta_{\kappa_{\omega}, \mu}$. Therefore for some $q^{* *} \leq q^{*}, q^{* *} \in \mathscr{P}$, and some term $\dot{\mathscr{A}}$ (the term is in $V^{S(B)}$ ) we have

$$
\begin{aligned}
q^{*} \Vdash \dot{\mathscr{A}} \subseteq \mu \wedge|\dot{\mathscr{A}}|<\kappa_{\omega}, \dot{\mathscr{A}} \text { is a subalgebra of } \tau, \\
\quad \text { and } \dot{S} \cap \dot{\mathscr{A}} \text { is stationary in } \sup (\dot{\mathscr{A}}) .
\end{aligned}
$$

But $\dot{\mathscr{A}}$ is a $\mathscr{P}$ term for a set of ordinals of cardinality less than $\kappa_{\omega}$. Again using $|\mathscr{P}|=\kappa_{\omega}^{+}$we can code $\dot{A}$ as a set of ordinals of cardinality $\leq \kappa_{\omega}^{+}$. Since $S(B)$ is $\kappa_{\omega}^{++}$distributive, this coded set is in $V$, so we can assume $\dot{A} \in V$. Now it is easily checked that also over $V$

$$
\begin{aligned}
q^{*} \Vdash \dot{\mathscr{A}} \subseteq \mu \wedge|\dot{\mathscr{A}}|<\kappa_{\omega} \wedge \dot{\mathscr{A}} \text { is a subalgebra of } \tau, \\
\text { and } \dot{S} \cap \dot{\mathscr{A}} \text { is stationary in } \sup (\dot{\mathscr{A}}) .
\end{aligned}
$$

Hence we proved that in $V$ we have $\Delta_{\kappa_{\omega}, \mu}$.

We shall now prove that in $V^{P} \kappa_{\omega}$ is fully compact. Actually we shall prove that $\Delta_{\kappa_{\omega}, \lambda}^{-}$holds in the model for every regular $\lambda>\kappa_{\omega}$, which by Theorem 2.3 is sufficient. So we are given in $V^{P}$ an algebra $\mathscr{A}$ on a regular cardinal $\lambda>\kappa_{\omega}$ and a stationary subset $S \subseteq \lambda$ where, for $\delta \in S, \operatorname{cf}(\delta)<\kappa_{\omega}$. We are also given, for $\delta \in S, D_{\delta} \subseteq \delta$ where $\left|D_{\delta}\right| \leq \kappa_{n}$ for some fixed $n$. If $\Delta_{\kappa_{\omega}, \lambda}$ holds, 
we get the conclusion of $\Delta_{\kappa_{\omega}, \lambda}^{-}$; hence by Lemma 16 we can assume $\lambda=\mu^{+}$ where $\mu$ is singular and $\operatorname{cf}(\mu)>\kappa_{\omega}^{+}$or that $\lambda$ is a non-Mahlo inaccessible. (In the last case we put $\mu=\lambda$.) Note that in the present case $\delta^{\kappa_{\omega}^{+}}<\lambda$ for every $\delta<\lambda$.

The set $S$, the algebra $\mathscr{A}$, and the assignment $\delta \rightarrow\left(D_{\delta}\right)$ are defined in $V^{\mathscr{P}}$, but since $\kappa_{\omega}^{+}<\lambda$ and $|\mathscr{P}|=\kappa_{\omega}^{+}$there is a stationary set $T \subseteq \lambda$ such that for some condition $p \in \mathscr{P}, p \Vdash T \subseteq S$. For $\delta \in T$ let $D_{\delta}^{*}$ be $\{\beta \mid \beta<\delta$ some $\left.q \leq p, q \Vdash \beta \in D_{\delta}\right\}$. Since $D_{\delta}$ is forced to have cardinality less than $\kappa_{\omega}$ and since $|\mathscr{P}|=\kappa_{\omega}^{+}$, we get $\left|D_{\delta}^{*}\right| \leq \kappa_{\omega}^{+}$.

$T$ and $\left\langle D_{\delta}^{*} \mid \delta \in T\right\rangle$ are now defined in $V$. We go back to arguing over $V$. Pick an adequate set $B$ such that $\left\{\kappa_{\omega}^{++}, \lambda^{+}\right\} \subseteq B$, and such that $\left\langle U_{n} \mid n<\omega\right\rangle$, $\left\langle f_{n} \mid n<\omega\right\rangle$ are good for $B$. Since $|B| \leq \kappa_{\omega}^{+}, B$ is not cofinal in $\mu$; hence if $\eta=\sup (B \cap \mu)$ we have that $\eta \in B, \eta<\mu, \eta$ is not the successor of a singular cardinal, and it is not a non-Mahlo inaccessible. Also we have $\bar{\eta}>\lambda$ (where $\bar{\eta}$ is the minimal member of $B$ above $\eta)$. Note that $\kappa_{\omega}^{+}<\eta$. Hence $\operatorname{Col}(\eta,<\bar{\eta})$ appears as one of the components in $S(B)$. In particular if we denote the Levy collapse that collapses $\lambda$ to have cardinality $\eta$ by $\operatorname{Col}(\eta, \lambda)$, there is a natural projection of $S(B)$ onto $\operatorname{Col}(\eta, \lambda)$, and this projection introduces a generic function $g: \eta \stackrel{\text { onto }}{\rightarrow} \lambda, g$ one-to-one. (Note that in $V^{S(B)} \eta$ is still a regular cardinal.) For $f \in S(B)$ let $f^{*}$ be its projection to $\operatorname{Col}(\eta, \lambda) . f^{*}$ is a function from a subset of $\eta$ (of cardinality less than $\eta$ ) into $\lambda$. Given $E \subseteq \lambda,|E|<\eta$, any condition $f$ can be extended to $f^{\prime}$ such that $E \subseteq \operatorname{range}\left(f^{\prime}\right)^{*}$. $\left(f^{*}\right.$ is partial information on the collapse map $g$.) Also every $f^{\prime}$ can be extended to $f$ so that domain $\left(f^{*}\right)$ is an ordinal less than $\eta$.

Lemma 17. In $V^{S(B)}$

$$
T^{*}=\left\{\beta \mid \beta<\eta, \sup g " \beta=\alpha \text { is in } T \text {, and } D_{\alpha}^{*} \subseteq g " \beta\right\}
$$

is a stationary subset of $\eta$.

Proof. Assume otherwise. Let $\tau$ be a term forced by some condition in $S(B)$, $f$, to denote a closed unbounded subset of $\eta$ disjoint from $T^{*}$.

Consider the structure (in $V) \mathscr{C}=\left\langle H_{\rho}, \in, S(B), \tau, \eta, \lambda\right\rangle$ where $\rho$ is any regular cardinal large enough so that $S(B), \tau \in H_{\rho}$. By induction on $\lambda$ we can easily define an increasing sequence of elementary substructures of $\mathscr{C}$, $\left\langle N_{\alpha} \mid \alpha<\lambda\right\rangle$, where $\left|N_{\alpha}\right|<\lambda, N_{\alpha} \cap \lambda$ is an ordinal (which is of course less than $\lambda), \alpha \subseteq N_{\alpha}, f \in N_{0},\left\langle N_{\beta} \mid \beta \leq \alpha\right\rangle \in N_{\alpha+1}, N_{\alpha}$ for limit $\alpha$ is $\bigcup_{\beta<\alpha} N_{\beta}$, and all subsets of $N_{\alpha}$ of cardinality $\leq \kappa_{\omega}^{+}$are in $N_{\alpha+1}$. (Namely $P_{\kappa_{\omega}^{++}}\left(N_{\alpha}\right) \subseteq N_{\alpha+1}$. We can satisfy this last condition because $\left|N_{\alpha}^{\kappa_{\omega}^{+}}\right|<\lambda$ by our assumptions about $\lambda$.

Since $\left\{\alpha \mid \alpha\right.$ limit $\left.N_{\alpha} \cap \lambda=\alpha\right\}$ form a closed unbounded subset of $\lambda$ and $T$ is stationary, we can find $\alpha \in T$ such that $N_{\alpha} \cap \lambda=\alpha$. Let $\zeta=\operatorname{cf}(\alpha)$. (Recall that $\left.\zeta<\kappa_{\omega}.\right)$ Fix a cofinal continuous sequence in $\alpha,\langle\beta(\delta) \mid \delta<\zeta\rangle$. By induction on $\zeta$ we define a decreasing sequence of conditions in $S(B) \cap N_{\alpha},\left\langle f_{\delta} \mid \delta<\zeta\right\rangle$, where, for $\gamma<\zeta,\left\langle f_{\delta} \mid \delta<\gamma\right\rangle \in N_{\alpha}, f_{\delta} \in N_{\beta(\delta)+1}$. (Actually $\left\langle f_{\delta} \mid \delta<\gamma\right\rangle \in$ 
$\left.N_{\beta_{\gamma+1}} \subseteq N_{\alpha}.\right) \quad \beta(\delta) \leq \sup \left(\right.$ range $\left.f_{\delta+1}^{*}\right)<\alpha, \eta_{\delta}=\operatorname{Dom}\left(f_{\delta}^{*}\right)$ is an ordinal less than $\eta$, and $f_{\delta+1}$ forces some ordinal between $\eta_{\delta}$ and $\eta_{\delta+1}$ to be in $\tau$. Also $D_{\alpha}^{*} \cap N_{\beta(\delta)} \subseteq \operatorname{range}\left(f_{\delta+1}\right)$.

$f_{0}$ is $f$. For limit $\gamma$ pick a condition below $\left\langle f_{\delta}\right| \delta\langle\gamma\rangle$; such a condition exists because $S(B)$ is $\kappa_{\omega}$ closed. We can pick this condition in $N_{\beta(\gamma)+1}$ because by the induction assumption $f_{\delta} \in N_{\beta(\delta)+1} \subseteq N_{\beta(\gamma)}$. Therefore $\left\langle f_{\delta}\right| \delta\langle\gamma\rangle \subseteq$ $N_{\beta(\gamma)+1}$. By our definition of the sequence $\left\langle N_{\nu} \mid \nu<\lambda\right\rangle$ (noting that $|\gamma|<\kappa_{\omega}$ ), $\left\langle f_{\delta} \mid \delta<\gamma\right\rangle \in N_{\beta(\gamma)+1}$, but $N_{\beta(\gamma)+1}$ is an elementary substructure of $\mathscr{C}$, so we can find the lower bound to $\left\langle f_{\delta}\right| \delta\langle\gamma\rangle$ in $N_{\beta(\gamma)+1}$. This lower bound will be $f_{\gamma}$. Without loss of generality we can assume that $\operatorname{Dom}\left(f_{\gamma}\right)$ is an ordinal $<\eta$ (which we denote by $\eta_{\gamma}$ ). For successor $\gamma=\delta+1$, we can find an extension of $f_{\delta}, h^{*}$, such that

(a) range $\left(h^{*}\right) \supseteq\left(D_{\alpha}^{*} \cap N_{\beta(\delta)}\right) \cup\{\beta(\delta)\}$.

(b) $h$ forces some ordinal above $\eta_{\delta}$ in $\tau$ and the domain of $h^{*}$ is above this ordinal.

Such an $h^{*}$ can be found in $N_{\beta(\delta)+2}$ because $\left|D_{\alpha}^{*}\right| \leq \kappa_{\omega}^{+}$; hence $D_{\alpha}^{*} \cap N_{\beta(\delta)} \in$ $N_{\beta(\delta)+1}$ and $\beta(\delta) \in N_{\beta(\delta)+1}, f_{\delta} \in N_{\beta(\delta)+1}$. Since $N_{\beta(\delta)+1} \subseteq N_{\beta(\delta)+2}$ and since they are elementary substructures of $\mathscr{C}$, we can find $h^{*}$ to be in $N_{\beta(\delta)+2}$ and define $f_{\gamma}$ to be this $h^{*}$.

Let $f_{\zeta}$ be a lower bound of $\left\langle f_{\delta} \mid \delta<\zeta\right\rangle$ which exists since $|\zeta|<\kappa_{\omega}$. Let $\eta_{\zeta}=\sup \left\langle\eta_{\delta} \mid \delta<\zeta\right\rangle$. Clearly $\eta_{\zeta}<\eta$. $f_{\lambda}$ forces that the range of $g$ on $\eta_{\zeta}$ is included in $N_{\alpha} \cap \lambda=\alpha$ (because each $f_{\delta} \in N_{\alpha}$ and the range of $f_{\delta}^{*}$ is also in $\left.N_{\alpha}\right) . g " \eta_{\zeta}$ is forced to be $\bigcup_{\delta<\zeta}$ range $\left(f_{\delta}\right)$, and we get that $\alpha=\sup _{\delta<\zeta} \beta(\delta) \leq$ $\sup g " \eta_{\zeta}$; hence $f_{\lambda} \Vdash \sup g " \eta_{\zeta}=\alpha . f_{\zeta}$ also forces that $D_{\alpha}^{*} \subseteq g " \eta_{\zeta}$ and that between $\eta_{\delta}$ and $\eta_{\delta+1}$ (for $\left.\delta<\zeta\right)$ there is an element of $\tau$. Since $\tau$ is forced to be closed, $f_{\lambda} \Vdash \eta_{\zeta} \in \tau$. But all the previous remarks show that $\eta_{\zeta} \in T^{*}$. We get a contradiction to $\tau$ being disjoint from $T^{*}$.

Exactly as in the proof of Lemma 16, we can show that the sequences $\left\langle\kappa_{\omega}\right| n<$ $\omega\rangle,\left\langle U_{n}\right| n\langle\omega\rangle$, and $\left\langle f_{n}\right| n\langle\omega\rangle$, and the cardinal $\eta$ satisfy in $V^{S(B)}$ all the assumptions of Theorem 3.15; hence

$$
V^{S(B) * \mathscr{D}} \vDash \Delta_{\kappa_{\omega}, \eta} .
$$

We assume that the condition $p \in \mathscr{P}$, mentioned above when we defined $T$, is in $G(\mathscr{P})$. In $V^{S(B) * \mathscr{P}}, T^{*}$ (defined in Lemma 17) is still a stationary subset of $\eta$ because $|\mathscr{P}|=\kappa_{\omega}^{+}\left\langle\eta\right.$. Also the sequence $\left\langle D_{\delta} \mid \delta \in T\right\rangle$ and the algebra $\mathscr{A}$ are in $V^{S(B) * \mathscr{P}}\left(V^{\mathscr{D}}\right.$ is included in $\left.V^{S(B) * \mathscr{P}}\right)$.

In $V^{S(B) * \mathscr{P}}$ consider an algebra $\mathscr{A}$ on $\lambda$ with $\kappa_{n}$ many operations (recall that we assumed that, for $\delta \in T,\left|D_{\delta}\right|<\kappa_{n}$ ), such that, for any subalgebra of $\mathscr{A}, \mathscr{B}$, if $\delta \in \mathscr{B} \cap T$ then $D_{\delta} \subseteq \mathscr{B}, \kappa_{n} \subseteq \mathscr{B}, \mathscr{B}$ is closed under $g$ and $g^{-1}$ ( $g$ is the generic function $g: \eta \stackrel{\text { onto }}{\rightarrow} \lambda$ ), and if $\alpha<\eta, \alpha \in \mathscr{B}$, then $\sup g^{\prime \prime} \alpha$ is in $\mathscr{B}$. 
Apply Lemma 2.4 in $V^{S(B) * \mathscr{D}}$ and get a subalgebra $\mathscr{B}$ of $\mathscr{A}$ of cardinality less than $\kappa_{\omega}$ such that the order type of $\mathscr{B} \cap \eta$ is a regular cardinal $\gamma<\kappa_{\omega}$ (clearly $\kappa_{n}<\gamma$ ) and $T^{*} \cap \mathscr{B}$ is stationary in $\sup \mathscr{B}$. The term denoting $\mathscr{B}$ in $V^{S(B)}$ can be easily coded as a set of ordinals of cardinality $\leq \kappa_{\omega}^{+}$, and since $S(B)$ is $\kappa_{\omega}^{++}$distributive, this term can be assumed to be in $V$. Hence $\mathscr{B}$ is in $V^{\mathscr{P}}$ (as well as $T^{*} \cap \mathscr{B}$, and $g\lceil\mathscr{B} \cap \eta)$. Now we argue in $V^{\mathscr{P}}$. Let $h$ be the unique order-preserving map from $\gamma$ onto $\mathscr{B} \cap \eta$. We get $R=\{\beta \mid \beta<\gamma$, $\left.h(\beta) \in T^{*} \cap \mathscr{B}\right\}$ is a stationary subset of $\gamma$. By our assumption the function $g \circ h$ is a one-to-one mapping from $\gamma$ onto $\mathscr{B}$. Note that the function on $\gamma, \kappa(\rho)=\sup g \circ h^{\prime \prime} \rho$, is continuous and for $\rho \in R$ is mapped into $\mathscr{B}$; hence $\widetilde{T}=g \circ h^{\prime \prime} R \subseteq T$ is a stationary in $\sup (\mathscr{B})$, and if we put, for $\delta \in \widetilde{T}$, $\mathscr{B}_{\delta}=g \circ h^{\prime \prime}\left((g \circ h)^{-1}(\delta)\right)$, then $\left|\mathscr{B}_{\delta}\right|<\gamma=|\mathscr{B}|$ and $\mathscr{B}=\bigcup_{\delta \in \tilde{T}} \mathscr{B}_{\delta}$. Also for $\delta \in \widetilde{T}$, it can be easily shown that $D_{\delta} \subseteq \mathscr{B}_{\delta}$. Let $x \in D_{\delta}$; then $x \in D_{\delta}^{*} \cap \mathscr{B}$, and hence $g^{-1}(x) \in \mathscr{B} \cap \eta$. Let $\bar{\delta}=g^{-1}(\delta)$. Since $\delta \in \widetilde{T}, \bar{\delta} \in T^{*}$. Also $\delta=\sup g^{\prime \prime} \bar{\delta}$ and $D_{\delta}^{*} \subseteq g^{\prime \prime} \bar{\delta}$. Therefore $g^{-1}(x) \in \bar{\delta} \cap \mathscr{B}=h^{\prime \prime}\left(h^{-1}(\bar{\delta})\right)=$ $\left.(g \circ h)^{\prime \prime}\left((g \circ h)^{-1}(\delta)\right)=\mathscr{B}_{\delta}\right)$. We get that $\mathscr{B}, \widetilde{T}$, and $\left\langle\mathscr{B}_{\delta} \mid \delta \in \widetilde{T}\right\rangle$ witness the truth of $\Delta_{\kappa_{\omega}, \lambda}^{-}$, so we have proved Theorem 1 .

\section{REFERENCES}

[Ba1] J. Baumgartner, A new class of order types, Ann. Math. Logic 9 (1976), 187-222.

[Ba2] Iterated forcing, Surveys in Set Theory (A. Mathias, ed.), London Math. Soc. Lecture Note Ser., no. 87, Cambridge Univ. Press, Cambridge, 1983, pp. 1-59.

[Bd] S. Ben-David, On Shelah compactness of cardinals, Israel J. Math. 31 (1978), 34-56; Corrigendum, 394.

[B-M] M. Burke and M. Magidor, Shelah Pcf theory and its applications, Ann. Pure Appl. Logic 50 (1990), 207-254.

[Cu-Wo] J. Cummings and H. Woodin, Applications of Radin's forcing (to appear).

[Ek] P. Eklof, On the existence of $\kappa$ free Abelian groups, Proc. Amer. Math. Soc. 47 (1975), 65-72.

[Ek-Me] P. Eklof and A. Mekler, Almost free modules, set theoretic methods, North-Holland, Amsterdam, 1990.

[E-R] P. Erdös and R. Rado, A partition calculus in set theory, Bull. Amer. Math. Soc. 62 (1956), 427-489.

[F-W] M. Foreman and W. H. Woodin, The Generalized Continuum Hypothesis can fail everywhere, Ann. of Math. (2) 133 (1991), 1-35.

[Fu] L. Fuchs, Infinite Abelian groups, Vols. I, II, Academic Press, New York, 1970, 1973.

[Gi] M. Gitik, The negation of the singular cardinal hypothesis from $o(\kappa)=\kappa^{++}$, Ann. Pure Appl. Logic 43 (1989), 209-234.

[Gr] P. Grifith, Infinite Abelian group theory, Univ. of Chicago Press, Chicago, IL, 1970.

[Hi] G. Higman, Almost free groups, Proc. London Math. Soc. 3 (1951), 284-290.

[Hil1] P. Hill, On the splitting of modules and Abelian groups, Canad. J. Math. 26 (1974), 68-77.

[Hil2] _ On the freeness of Abelian groups, A generalization of Pontryagin's theorem, Bull. Amer. Math. Soc. 76 (1970), 1118-1120.

[Hil3] _ A special criterion for freeness, Sympos. Math. 13 (1974), 311-314. 
[Ho] W. Hodges, In singular cardinality locally free algebras are free, Algebra Universalis 12 (1981), 205-220.

[Je] T. J. Jech, Set theory, Academic Press, New York, San Francisco, and London, 1978.

[Jen] R. B. Jensen, The fine structure of the constructible universe, Ann. Math. Logic 4 (1972), 229-308.

[K-M] A. Kanamori and M. Magidor, The development of large cardinals axioms in set theory, Higher Set Theory (G. Muller, ed.), Lecture Notes in Math., vol. 669, Springer-Verlag, Berlin and New York, 1978.

[La] R. Laver, Making the supercompact $\kappa$ indestructible under $\kappa$ directed closed forcing, Israel J. Math. 29 (1978), 385-388.

[Ma] M. Magidor, On the singular cardinals problem. I, Israel J. Math. 28 (1977), 1-31.

[Me] A. Mekler, How to construct almost free groups, Canad. J. Math. 32 (1980), 1206-1228.

[Men] T. K. Menas, A combinatorial property of $P_{\kappa}(\lambda)$, J. Symbolic Logic 41 (1976), 225-233.

[Mi-Sh] E. C. Milner and S. Shelah, Some theorems on transversals, Infinite and Finite Sets, a Book Dedicated to P. Erdös on His 60th Birthday, North-Holland, Amsterdam, 1975, pp. 1115-1126.

[Sh1] S. Shelah, A compactness theorem for singular cardinals, free algebras, Whitehead problem and transversals, Israel J. Math. 21 (1975), 319-339.

[Sh2] _ On successors of singular cardinals, Logic Colloquium 78 (M. Boffa, D. Van Dallen, and K. McAloon, eds.), North-Holland, Amsterdam, 1979, pp. 357-380.

[Sh3] _ Proper forcing, Lecture Notes in Math., vol. 940, Springer-Verlag, Berlin, Heidelberg, and New York, 1982.

[Sh4] _ Incompactness in regular cardinals, Notre Dame J. Formal Logic 26 (1985), 195-228.

[Sh5] _ Cardinal arithmetics, Oxford Univ. Press (to appear).

[S-R-K] R. M. Solovay, W. N. Rienhardt, and A. Kanamoi, Strong axioms of infinity and elementary embeddings, Ann. Math. Logic 13 (1978), 73-116.

ABstract. We show that the construction of an almost free nonfree Abelian group can be pushed from a regular cardinal $\kappa$ to $\kappa_{\kappa+1}$. Hence there are unboundedly many almost free nonfree Abelian groups below the first cardinal fixed point.

We give a sufficient condition for " $\kappa$ free implies free", and then we show, assuming the consistency of infinitely many supercompacts, that one can have a model of ZFC+G.C.H. in which $\aleph_{\omega^{2}+1}$ free implies $\aleph_{\omega^{2}+2}$ free. Similar construction yields a model in which $\aleph_{\kappa}$ free implies free for $\kappa$ the first cardinal fixed point (namely, the first cardinal $\alpha$ satisfying $\alpha=\aleph_{\alpha}$ ). The absolute results about the existence of almost free nonfree groups require only minimal knowledge of set theory. Also, no knowledge of metamathematics is required for reading the section on the combinatorial principle used to show that almost free implies free. The consistency of the combinatorial principle requires acquaintance with forcing techniques.

Institute of Mathematics, Hebrew University, Jerusalem, IsRael 